\title{
Properties of Noncommutative Rényi and Augustin Information
}

\author{
Hao-Chung Cheng ${ }^{1,2,3}$, Li GaO ${ }^{4}$ and Min-Hsiu Hsieh ${ }^{3}$ \\ ${ }^{1}$ Department of Electrical Engineering 83 Graduate Institute of Communication Engineering \\ ${ }^{2}$ Department of Mathematics 83 Institute of Applied Mathematical Sciences \\ National Taiwan University, 106 Taipei, Taiwan (R.O.C.) \\ ${ }^{3}$ Hon Hai (Foxconn) Quantum Computing Centre, New Taipei City 236, Taiwan (R.O.C.) \\ ${ }^{4}$ Department of Mathematics, University of Houston, TX 77004, United States
}

\begin{abstract}
Rényi and Augustin information are generalizations of mutual information defined via the Rényi divergence, playing a significant role in evaluating the performance of information processing tasks by virtue of its connection to the error exponent analysis. In quantum information theory, there are three generalizations of the classical Rényi divergence - the Petz's, sandwiched, and log-Euclidean versions, that possess meaningful operational interpretation. However, the associated quantum Rényi and Augustin information are much less explored compared with their classical counterpart, and lacking crucial properties hinders applications of these quantities to error exponent analysis in the quantum regime.

The goal of this paper is to analyze fundamental properties of the Rényi and Augustin information from a noncommutative measure-theoretic perspective. Firstly, we prove the uniform equicontinuity for all three quantum versions of Rényi and Augustin information, and it hence yields the joint continuity of these quantities in order and prior input distributions. Secondly, we establish the concavity of the scaled Rényi and Augustin information in the region of $s \in(-1,0)$ for both Petz's and the sandwiched versions. This completes the open questions raised by Holevo [IEEE Trans. Inf. Theory, 46(6):2256-2261, 2000], and Mosonyi and Ogawa [Commun. Math. Phys., 355(1):373-426, 2017]. For the applications, we show that the strong converse exponent in classical-quantum channel coding satisfies a minimax identity, which means that the strong converse exponent can be attained by the best constant composition code. The established concavity is further employed to prove an entropic duality between classical data compression with quantum side information and classical-quantum channel coding, and a Fenchel duality in joint source-channel coding with quantum side information.
\end{abstract}

\section{INTRODUCTION}

Error exponent analysis aims at evaluating the exponential behavior of the performance (e.g. the error probability or success probability) of the underlying system when certain size or rate is fixed. Early studies can be found in hypothesis testing, detection and estimation theory, and varieties of statistical applications $[1,2,3,4,5,6,7,8,9,10,11,12,13]$. It is arguably a substantial research topic in information theory because the analysis can be viewed as a refinement of Shannon's seminal source coding and channel coding theorem [14]. In this paper, we focus on the problems of information transmission or the so-called channel coding. Let $\mathcal{W}: x \mapsto W_{x}$ be a probabilistic channel that maps symbols from the input alphabet $\mathcal{X}$ to an measurable output space. The goal of a communication system is to design a good coding strategy for $n$ uses of the channel that minimizes the error probability of decoding. Drawing a connection to the large deviation principle [13], the optimal exponent given a fixed transmission rate $R$ is determined by the Fenchel-Legendre transform of the scaled Rényi information $E_{0}^{\mathrm{r}}(s, P)$ (maximized over all priors $P$ )

E-mail address: haochung.ch@gmail.com, gaolimath@gmail.com, minhsiuh@gmail.com.

Date: May 31, 2022. 
$[15,16,17,18,19,20,21,19,22,23,24,25]:^{1}$

$$
\begin{aligned}
& \lim _{n \rightarrow \infty}-\frac{1}{n} \log \varepsilon^{\star}(n, R)=\sup _{0 \leq s \leq 0} \sup _{P}\left\{E_{0}^{r}(s, P)-s R\right\}, \quad R<C_{\mathcal{W}} \\
& \lim _{n \rightarrow \infty}-\frac{1}{n} \log \left[1-\varepsilon^{\star}(n, R)\right]=\sup _{-1<s<0} \inf _{P}\left\{E_{0}^{\mathbf{r}}(s, P)-s R\right\}, \quad R>C_{\mathcal{W}}
\end{aligned}
$$

where $\varepsilon^{\star}(n, R)$ denotes the optimal error probability; $C_{\mathcal{W}}$ is the channel capacity; and $E_{0}^{\mathrm{r}}(s, P)$ for a prior probability mass function $P$ is called the auxiliary function introduced by Gallager $[18,19]$ :

$$
E_{0}^{\mathrm{r}}(s, P):=-\log \int\left(\sum_{x} P(x)\left(\frac{\mathrm{d} W_{x}}{\mathrm{~d} \nu}\right)^{\frac{1}{1+s}}\right)^{1+s} \mathrm{~d} \nu, \quad s>-1,
$$

where $\nu$ is any reference measure ${ }^{2}$ such that $W_{x}$ is absolutely continuous with respect to $\nu$ for all $x$ with $P(x)>0$.

The auxiliary function presented above has a close relation to a one-parameter generalization of Shannon's mutual information. Sibson [27] introduced one candidate in terms of Rényi's divergence $D_{\alpha}[28,29]$ and showed that it equals a scaled version of Gallager's auxiliary function:

$$
\begin{aligned}
I_{\alpha}^{\mathrm{r}}(P, \mathcal{W}) & :=\inf _{q} D_{\alpha}(P \circ \mathcal{W} \| P \otimes q) \\
& =\left.\frac{E_{0}^{\mathrm{r}}(s, P)}{s}\right|_{s=\frac{1-\alpha}{\alpha}},
\end{aligned}
$$

where the infimum is taken over all probability measures $q$ on the output measurable space, and $P \circ \mathcal{W}$ denotes the joint probability measure on the product of input and output spaces. We termed $I_{\alpha}^{\mathrm{r}}(P, \mathcal{W})$ the order- $\alpha$ Rényi information for a prior $P$.

Augustin [30] and Csiszár [31] proposed another generalization ${ }^{3}$ of Shannon's mutual information, which is termed as the order- $\alpha$ Augustin information for a prior $P$ [30, 31, 35, 36]:

$$
I_{\alpha}^{\mathrm{a}}(P, \mathcal{W}):=\inf _{q} \sum_{x} P(x) D_{\alpha}\left(W_{x} \| q\right) .
$$

When maximizing over all priors $P$, both Rényi information and Augustin information equal the order- $\alpha$ Rényi capacity [37, 31, 38, 36]:

$$
C_{\alpha, \mathcal{W}}:=\sup _{P} I_{\alpha}^{\mathrm{r}}(P, \mathcal{W})=\sup _{P} I_{\alpha}^{\mathrm{a}}(P, \mathcal{W})
$$

One can define the auxiliary function associated with the Augustin information by drawing inspiration from Eq. (5):

$$
E_{0}^{\mathrm{a}}(s, P):=s I_{\frac{1}{1+s}}^{\mathrm{a}}(P, \mathcal{W})
$$

Similar to the role of $E_{0}^{\mathrm{r}}(s, P)$ in Eqs. (1) and (2), the Fenchel-Legendre transform of $E_{0}^{\mathrm{a}}(s, P)$ was shown to be equal to the optimal exponent of channel coding with constant composition codes [39, 40, 41, 42, $30,43,44,45,23,46,31,47,26,48,49,50]$.

In addition to the channel coding problems, the connections of the auxiliary functions ${ }^{4}$ to the exponents in other information tasks, e.g. source coding and channel coding networks, have been established as well $[57,58,59,60,46,61,62,63,64]$. This justifies the operational significance of the auxiliary functions in

\footnotetext{
${ }^{1}$ More precisely, Eq. (1) was proven for any fixed rate below the channel capacity and above the critical rate [18, 20, 19, 26], the rate at which the slope of the right-hand side of (1) is -1. Recently, Nakiboğlu in [25, Lemma 29] showed that Eq. (1) holds for any fixed rate greater than $C_{\frac{1}{1+L}}, \mathcal{W}$ under list decoding [26] with list size $L \in\{1, \ldots, M-1\}$, where $M$ is the size of the message set.

${ }^{2}$ Note that the quantity $E_{0}^{\mathrm{r}}(s, P)$ does not depend on the choice of the reference measure.

${ }^{3}$ There is another version defined by Arimoto [32]. After maximizing over all priors, the three quantities correspond to the order- $\alpha$ Rényi capacity in Eq. (6). However, we omit the discussion of this version due to its limited uses. We refer the readers' to the comparison by Verdú [33] and by Aishwarya and Madiman[34].

${ }^{4}$ The auxiliary functions in different protocols are defined in a slightly different but similar way [51, 52, 53, 54, 55, 56]. We refer the readers to Section 6 for further discussion.
} 
information theory. Therefore, understanding their properties is of substantial interest and allows us to better characterize the performance of the information tasks. Early works on the continuity properties were done by Gallager [18, p. 28], Shannon, Gallager, and Berlekamp [20, p. 101], and Csiszár and Körner $[65,26]$. The first-order and second-order derivatives at $s=0$ correspond, respectively, to Shannon's mutual information and information variance [19, p. 142], [66, Lemma 1]. Those properties are critical to high-order analysis in the finite blocklength regime [67, 68, 69, 49, 70] and moderate deviation analysis $[66,53]$. The concavity of $E_{0}^{r}(s, P)$ in $s>-1$ was first proved by Gallager [19, Theorem 5.6.3] using Hölder's inequality. Essentially, the concavity of $E_{0}^{r}$ is equivalent to Littlewood's version of Hölder's inequality $^{5}$ [71, Theorem 5.5.1]. As for $E_{0}^{\mathrm{a}}(s, P)$, Csiszár [31, (A24), (A27)] (see also [29, Theorem 30], [72]) proved a variational representation for the case of finite-dimensional output spaces:

$$
E_{0}^{\mathrm{a}}(s, P)=\inf _{\mathcal{V}}\left\{\sum_{x} P(x) D\left(V_{x} \| W_{x}\right)+s I(P, \mathcal{V})\right\}, \quad s>-1,
$$

where the infimum is taken over all dummy channels on the same input and output spaces of $\mathcal{W} ; I(P, \mathcal{V})$ is Shannon's mutual information; and $D(\cdot \| \cdot)$ is the Kullback-Leibler divergence. Then, the concavity in $s>-1$ immediately follows because a pointwise infimum of linear functions is concave. We remark that the concavity property in $s$ has numerous usefulness. For example, it determines the convexity and decreases of the entropic quantities in $R$ [19, p. 142], and it is indispensable in proving the saddle-point property in sphere-packing exponents [73, 52, 54], and the moderate deviations [66]. The properties of the auxiliary functions can also be derived via those of the Rényi and Augustin information. We refer the readers to the review literature by Ho and Verdú [74, 33], Dalai [35], and the excellent expositions by Nakiboğlu [38, 36] from a measure-theoretic aspect.

In classical information theory, the (channel) output space consists of probability measures. It can be extended to more general noncommutative measure spaces, i.e. von Neumann algebras, as any quantummechanical system can be modeled by a density operator. One prominent example is the classicalquantum channel coding, where the output space contains density matrices [75, 76, 77, 78]. Therefore, one of the main aims of the current paper is to investigate the properties of the auxiliary functions using noncommutative $L_{p}$-theory. Moreover, the established results could be employed to perform refined analysis in quantum information processing tasks [52, 53, 54, 55, 56, 79].

There are at least three quantum generalizations of the classical Rényi divergence [28]: Petz's Rényi divergence $D_{\alpha}$ [80], the sandwiched Rényi divergence $D_{\alpha}^{*}[81,82,83,84]$, and the log-Euclidean Rényi divergence $D_{\alpha}^{b}[85,86]$. The quantum auxiliary functions are defined accordingly: for $(t)=\{\}, *$ or $b$,

$$
\begin{aligned}
& E_{0}^{\mathrm{r},(t)}(s, P):=s I_{\frac{1}{1+s}}^{\mathrm{r},(t)}(P, \mathcal{W}), \\
& E_{0}^{\mathrm{a},(t)}(s, P):=s I_{\frac{1}{1+s}}^{\mathrm{a},(t)}(P, \mathcal{W}) .
\end{aligned}
$$

Due to the noncommutative nature, it is generally more difficult to derive properties for them. Furthermore, there are no closed-form expressions except for $E_{0}^{r}(s, P)$. Actually, the three versions inherit different properties of the classical function. A quantum Sibson's identity holds for the Petz's version as in Eq. (5) [87]; the sandwiched version relates to weighted noncommutative $L_{p}$-norms; and the $\log$-Euclidean version satisfies the variational representation as in Eq. (7). We will exploit these facts in our derivations later.

Burnashev and Holevo [88, 89] first generalized Gallager's expression in Eq. (3) to a quantum auxiliary function:

$$
E_{0}^{r}(s, P):=-\log \operatorname{Tr}\left[\left(\sum_{x} P(x) W_{x}^{\frac{1}{1+s}}\right)^{1+s}\right]
$$

\footnotetext{
${ }^{5}$ There are several versions of Hölder's inequality. The one used by Gallager $[19, \quad(5 \mathrm{~B} .10)]$ is $\sum_{j} a_{j} b_{j} \leq$ $\left(\sum_{j} a_{j}^{1 /(1-\theta)}\right)^{1-\theta}\left(\sum_{j} b_{j}^{\theta}\right)^{\theta}$ for all $a_{j}, b_{j} \geq 0$ and $\theta \in[0,1]$. On the other hand, Littlewood's version, which is also called interpolation inequality, states that $\|\mathbf{u}\|_{1 /((1-\theta) p+\theta q)} \leq\|\mathbf{u}\|_{1 / p}^{1-\theta}\|\mathbf{u}\|_{1 / q}^{\theta}$, where $\|\mathbf{u}\|_{p}:=\left(\sum_{j} w_{j} u_{j}^{p}\right)^{1 / p}$ is the $p$-norm for nonnegative $\left(w_{j}\right)_{j}$.
} 
where the $\left\{W_{x}\right\}_{x}$ is a set of density operators in the output space. Sharma and Warsi [87] proved a quantum Sibson's identity to show that the expressions in Eqs. (4) and (5) are equal to Petz's version $E_{0}^{r}(s, P)$. If the density operators are all rank-one (i.e. pure-state channels), Burnashev and Holevo $[88,89]$ proved a random coding bound (i.e. achievability) on the optimal error probability in terms of the Fenchel-Legendre transform of $E_{0}^{\mathrm{r}}(s, P)$. Burnashev and Holevo [88, 89] also conjectured that their result holds when the output space consists of general density operators. Hayashi proved an achievability bound with a sub-optimal auxiliary function [90, 51]. Recently, Qi et al. extended Hayashi's expression to entanglement-assisted classical communications over quantum channels [91]. The sphere-packing bound (i.e. optimality) was first studied by Winter [92], and he proved the bound with the log-Euclidean version. Recently, Dalai [93] and part of the present authors [52] established a sphere-packing bound for all codes with Petz's version when maximizing over all priors $P$ as in the Eq. (1). The sphere-packing bound for constant composition codes was also proved by using $E_{0}^{\mathrm{a}}(s, P)$ [94, 52]. Compared with Winter's result, Petz's version is tighter than the log-Euclidean when $R<C_{\mathcal{W}}$ by Golden-Thompson's inequality $[95,96,97,35,52]$. In the strong converse regime $\left(R>C_{\mathcal{W}}\right)$, Mosonyi and Ogawa [86] proved that the strong converse exponent is determined by the sandwiched version, see Eq. (2) with $E_{0}^{\mathrm{r}, *}(s, P)$.

Regarding the properties of the auxiliary functions, Holevo [89] conjectured that $E_{0}^{\mathrm{r}}(s, P)$ is concave as in the classical case. Later, Fujii and Yanagi proved the concavity in the region $s \in[0,1]$ by directly analyzing the second-order derivatives. Part of the authors [98] employed a technique - the concavity of matrix geometric means - to show the concavity for all $s \geq 0$. Mosonyi and Ogawa in [86, Theorem 3.6, Lemma 5.13] showed that the log-Euclidean version satisfies the variational representation as in Eq. (7), the concavity of $E_{0}^{r, b}(s, P)$ and $E_{0}^{\mathrm{a}, b}(s, P)$ on $s>-1$ thus holds [86, Proposition B.5]. Most importantly, Mosonyi and Ogawa [86, Proposition B.1] showed that

$$
\alpha \mapsto(\alpha-1) D_{\alpha}^{(t)} \text { is convex on }(0,1) \text { implies that } s \mapsto E_{0}^{\mathbf{r},(t)}(s, P) \text { or } E_{0}^{\mathrm{a},(t)}(s, P) \text { is concave on }(0, \infty) \text {. }
$$

Since the convexity assumption is true by [86, Lemma 3.12], the concavity on $s \geq 0$ for all the versions was proved. However, the concavity for Petz's versions $E_{0}^{\mathrm{r}}(s, P)$ and $E_{0}^{\mathrm{a}}(s, P)$, and the sandwiched versions $E_{0}^{\mathrm{r}, *}(s, P)$ and $E_{0}^{\mathrm{a}, *}(s, P)$ on $s \in(-1,0)$ remains unknown.

The main contribution of this paper is proving the continuity of the quantum auxiliary functions and completing the last missing part of the concavity. First, we show that the finiteness of the order- $\alpha$ Rényi capacity implies the uniform equicontinuity of the Rényi information and Augustin information in prior, respectively, in the region $(0, \min \{1, \alpha\}]$ for Petz's version, in $(0, \alpha]$ for log-Euclidean version, and in $[1 / 2, \alpha]$ for sandwiched version (Propositions 4 and 5). Combining with the continuity of the Rényi information and Augustin information in their orders, we thus prove the joint continuity of the auxiliary functions in the argument (Theorem 13). Second, we establish the concavity property of the auxiliary functions on $s \in(-1,0)$ for both the Petz's and sandwiched versions (Theorems 11 and 12), which solves the open problems raised by Holevo [89], Mosonyi and Ogawa [86]. Moreover, the concavity results hold for the densities from any finite von Neumann algebras.

In order to prove the concavity, our main technique is the complex interpolation for noncommutative $L_{p}$ spaces. Firstly, we show that the Rényi auxiliary function for the sandwiched version, i.e. $E_{0}^{r, *}(s, P)$ is related to the amalgamated $L_{p}$-norm introduced by Junge and Parcet [99] (see also the vector-valued $L_{p}$ space norm by Pisier [100]). Its concavity in $s \in(-1,0)$ can be derived from complex interpolation of the amalgamated $L_{1}^{p}(\mathcal{N} \subset \mathcal{M})$-norm that for a positive $\rho \in \mathcal{M}$,

$$
\|\rho\|_{L_{1}^{p}(\mathcal{N} \subset \mathcal{M})}=\inf _{\sigma \in \mathcal{S}(\mathcal{N})}\left\|\sigma^{\frac{1-p}{2 p}} \rho \sigma^{\frac{1-p}{2 p}}\right\|_{p}, \quad p \in[1, \infty)
$$

Here $\mathcal{M}$ is a semifinite von Neumann algebra ${ }^{6} ; \mathcal{S}(\mathcal{N})$ denotes all density operators in the subalgebra $\mathcal{N} \subset \mathcal{M}$; and $\|\cdot\|_{p}$ is the $L_{p}$-norm on $\mathcal{M}$. The interpolation relation was proved in [99] $]^{7}$. Secondly, the concavity of the Augustin auxiliary function for the sandwiched version follows from an interpolation type

\footnotetext{
${ }^{6}$ The readers not familiar with von Neumann algebras can think $\mathcal{M}=\mathcal{B}(\mathcal{H})$ the bounded operators on a Hilbert space

${ }^{7}$ See Eq. (14) in Section 3 for the more detailed expression.
} 
inequality: the log-convexity of the map

$$
\frac{1}{p} \mapsto \inf _{\sigma \in \mathcal{S}(\mathcal{H})}\left\|\left(\sigma^{\otimes n}\right)^{\frac{1-p}{2 p}} \rho\left(\sigma^{\otimes n}\right)^{\frac{1-p}{2 p}}\right\|_{p}, \quad p \in[1, \infty), \rho \in \mathcal{B}(\mathcal{H})^{\otimes n} .
$$

Here the infimum is no longer taken over all density operators in a subalgebra $\mathcal{N}$ as in Eq. (8) but over all tensor-product states. This interpolation type inequality, i.e. the log-convexity given in (9), is shown in Theorem 11.

Regarding the auxiliary functions of Petz form, for $E_{0}^{\mathrm{r}}(s, P)$ we require the log-convexity of the map

$$
\frac{1}{p} \mapsto \inf _{\sigma \in \mathcal{S}(\mathcal{H})}\left(\operatorname{Tr}\left[\left(\sum_{x \in \mathcal{X}} P(x) W_{x}^{p}\right) \sigma^{1-p}\right]\right)^{\frac{1}{p}}, \quad p \in[1, \infty) .
$$

This quantity is related to a new noncommutative Sibson's identity (Proposition 2) and shows that the Rényi auxiliary function of Petz's form admits a representation $\operatorname{Tr}\left[\left(\mathbb{E}\left(\rho^{p}\right)\right)^{1 / p}\right]$, where $\mathbb{E}: \mathcal{M} \rightarrow \mathcal{N}$ is the conditional expectation. The concavity of $E_{0}^{r}(s, P)$ can be derived from the log-convexity of the map (Proposition 3)

$$
\frac{1}{p} \mapsto \operatorname{Tr}\left[\left(\mathbb{E}\left(\rho^{p}\right)\right)^{\frac{1}{p}}\right], \quad p \in[1, \infty), \rho \in \mathcal{M},
$$

For the Augustin auxiliary function of Petz's form $E_{0}^{\mathrm{a}}(s, P)$, we prove the log-convexity of the map

$$
\frac{1}{p} \mapsto \inf _{\sigma \in \mathcal{S}(\mathcal{H})}\left(\operatorname{Tr}\left[\rho^{p}\left(\sigma^{\otimes n}\right)^{1-p}\right]\right)^{\frac{1}{p}}, \quad p \in[1, \infty), \rho \in \mathcal{B}(\mathcal{H})^{\otimes n}
$$

The possible and future applications of the established results are the following. The joint continuity is useful in higher-order analysis in finite blocklength regime, and the variable-length classical data compression with quantum side information (also called the classical-quantum Slepian-Wolf source coding) [55]. For $s \in(-1,0)$, the auxiliary functions $E_{0}^{\mathrm{r},(t)}(s, P)$ and $E_{0}^{\mathrm{a},(t)}(s, P)$ are quasi-convex in prior $P$ by Propositions 4 and 5 . Hence, the established concavity in $s \in(-1,0)$ together with Sion's minimax theorem [101] immediately implies a minimax identity for the strong converse exponent:

$$
\begin{aligned}
& \sup _{-1<s<0} \inf _{P}\left\{E_{0}^{\mathrm{r}, *}(s, P)-s R\right\}=\inf _{P} \sup _{-1<s<0}\left\{E_{0}^{\mathrm{r}, *}(s, P)-s R\right\} \\
= & \sup _{-1<s<0} \inf _{P}\left\{E_{0}^{\mathrm{a}, *}(s, P)-s R\right\}=\inf _{P} \sup _{-1<s<0}\left\{E_{0}^{\mathrm{a}, *}(s, P)-s R\right\} .
\end{aligned}
$$

Moreover, the concavity is critical in proving an entropic duality between the classical data compression with quantum side information and a classical-quantum channel coding [55], and a Fenchel duality in joint source-channel coding with quantum side information [56]. We provide the comparisons of different notions of the auxiliary functions in Table 1.

The paper is organized as follows. In Section 2, we introduce the definition and notation for various quantum entropic quantities and the corresponding auxiliary functions. Section 3 reviews the basics of complex interpolation and proves an interpolation inequality. We prove several properties of the auxiliary functions in Section 5. In Section 6, we discuss their applications in quantum information theory. Finally, we conclude this paper in Section 7.

\section{Preliminaries and Notation}

Throughout this paper, we consider a complex separable Hilbert space $\mathcal{H}$, and let $|\mathcal{H}|$ denote its dimension. Let $\mathcal{B}(\mathcal{H})$ and $\mathcal{L}_{>0}(\mathcal{H})$ denote the algebra of bounded linear operators and non-zero positive

semi-definite operators on $\mathcal{H}$. For $0<p \leq \infty$, we denote by $\|M\|_{p}:=\left(\operatorname{Tr}|M|^{p}\right)^{1 / p}$ the Schatten $p$-norm for $\mathcal{B}(\mathcal{H})$ and also the $L_{p}$-norm for a von Neumann algebra $(\mathcal{M}, \operatorname{Tr})$. The Schatten $p$ class on $\mathcal{H}$ is denoted by $S_{p}(\mathcal{H}):=\left\{M \in \mathcal{B}(\mathcal{H}):\|M\|_{p}<\infty\right\}$. We use $\mathcal{S}(\mathcal{H})$ to denote the set of density operators (i.e. positive semi-definite operators with unit trace) on $\mathcal{H}$. We also use $\mathcal{S}(\mathcal{M})$ to denote the density operators of a von Neumann algebra $(\mathcal{M}, \operatorname{Tr})$ equipped with the trace Tr. Namely, $\mathcal{S}(\mathcal{M})$ is a subset of $L_{1}$-space $L_{1}(\mathcal{M})$ consisting of all positive and unit trace operators. Here we have slightly abused the notation that $\mathcal{S}(\mathcal{M})$ is the (normal) state space of von Neumann algebra $\mathcal{M}$, while the notation $\mathcal{S}(\mathcal{H}):=\mathcal{S}(\mathcal{B}(\mathcal{H})$ ) means the 


\begin{tabular}{|c|c|c|c|c|c|c|c|}
\hline Setting & Range of $s$ & Positivity & Monotone & Concave in $s$ & Continuity & $\left.\frac{\partial}{\partial s}\right|_{s=0}$ & $-\left.\frac{\partial^{2}}{\partial s^{2}}\right|_{s=0}$ \\
\hline$E_{0}^{r,(t)}(s, P)$ & $\begin{array}{c}{[-1,0)} \\
{[0, \infty]}\end{array}$ & $\begin{array}{l}<0 \\
\geq 0\end{array}$ & $\nearrow$ & $\cap$ & $\checkmark$ & $I(P, \mathcal{W})$ & $U^{(t)}(P, \mathcal{W})$ \\
\hline$E_{0}^{\mathrm{a},(t)}(s, P)$ & $\begin{array}{c}{[-1,0)} \\
{[0, \infty]}\end{array}$ & $\begin{array}{l}<0 \\
\geq 0\end{array}$ & $\nearrow$ & $\cap$ & $\checkmark$ & $I(P, \mathcal{W})$ & $V^{(t)}(P, \mathcal{W})$ \\
\hline$E_{0, \mathrm{~s}}^{(t)}(s)$ & $\begin{array}{c}{[-1,0)} \\
{[0, \infty]}\end{array}$ & $\begin{array}{l}\geq 0 \\
<0\end{array}$ & $\searrow$ & $\cap$ & $\checkmark$ & $-H(X \mid B)_{\rho}$ & $V^{(t)}(X \mid B)_{\rho}$ \\
\hline$E_{0, \mathrm{~s}}^{(t)}(s, P)$ & $\begin{array}{l}{[-1,0]} \\
{[0, \infty]}\end{array}$ & $\begin{array}{l}\geq 0 \\
<0\end{array}$ & ${ }^{\times}$ & $\cap$ & $\checkmark$ & $-H(X \mid B)_{\rho}$ & $V^{(t)}(P, \mathcal{W})$ \\
\hline
\end{tabular}

TABLE 1. The table compares properties of different types of the auxiliary functions in finite-dimensional Hilbert space. The functions $E_{0}^{\mathrm{r},(t)}(s, P), E_{0}^{\mathrm{a},(t)}(s, P), E_{0, \mathrm{~s}}^{(t)}(s)$, and $E_{0, \mathrm{~s}}^{(t)}(s, P)$, respectively, correspond to the auxiliary functions of Rényi information, Augusting information in classical-quantum channel coding, and the auxiliary functions in classical data compression with quantum side information of i.i.d. sources [54], and type-dependent sources [55]. The three values of $(t)=\{\}, *$, and b denote the Petz's, sandwiched, and logEuclidean Rényi divergence. In the last two columns, we assume the auxiliary functions are second-order differentiable with respect to $s$. The information variance quantities are defined by $V^{(t)}(P, \mathcal{W})=\sum_{x \in \mathcal{X}} P(x) V^{(t)}\left(W_{x} \| P \mathcal{W}\right), U^{(t)}(P, \mathcal{W})=V^{(t)}(P \circ \mathcal{W} \| P \otimes P \mathcal{W})$, and $V^{(t)}(X \mid B)_{\rho}=V^{(t)}\left(\rho_{X B} \| \mathbb{1}_{X} \otimes \rho_{B}\right)$. We refer the readers to Sections 2 and 5 for detailed definitions.

(normal) state space of $\mathcal{B}(\mathcal{H})$, which is a subset of the trace class operator $S_{1}(\mathcal{H})$ but not a subset of the Hilbert space $\mathcal{H}$. The symbol $\mathbb{1}_{\mathcal{H}}$ denotes the identity operator in $\mathcal{B}(\mathcal{H})$. We denote by $\mathcal{P}(\mathcal{X})$ the set of all probability measures on a finite set $\mathcal{X}$. For two real-valued functions $f$ and $g, f \vee g$ is the pointwise maximum of $f$ and $g$, and $f \wedge g$ is the pointwise minimum. We use $\operatorname{supp}(A)$ to denote the support of an operator or a function $A$. We use i to denote the imaginary unit.

2.1. Quantum Entropies. For $\rho, \sigma \in \mathcal{L}_{>0}(\mathcal{H})$ and $\alpha \in(0, \infty) \backslash\{1\}$, the Petz's Rényi divergence [80], sandwiched Rényi divergence [81, 82, 83, 84], and log-Euclidean Rényi divergence [85, 86] are defined as ${ }^{8}$

$$
\begin{aligned}
& D_{\alpha}(\rho \| \sigma):=\frac{1}{\alpha-1} \log \frac{\operatorname{Tr}\left[\rho^{\alpha} \sigma^{1-\alpha}\right]}{\operatorname{Tr}[\rho]}, \\
& D_{\alpha}^{*}(\rho \| \sigma):=\frac{1}{\alpha-1} \log \frac{\operatorname{Tr}\left[\left(\sigma^{\frac{1-\alpha}{2 \alpha}} \rho \sigma^{\frac{1-\alpha}{2 \alpha}}\right)^{\alpha}\right]}{\operatorname{Tr}[\rho]}, \\
& D_{\alpha}^{b}(\rho \| \sigma):=\frac{1}{\alpha-1} \log \frac{\operatorname{Tr}\left[\mathrm{e}^{\alpha \log \rho+(1-\alpha) \log \sigma}\right]}{\operatorname{Tr}[\rho]} .
\end{aligned}
$$

It is known [102, 81, 82] that all the $\alpha$-Rényi divergences converge to the Umegaki relative entropy [103]

$$
D(\rho \| \sigma):=\frac{1}{\operatorname{Tr}[\rho]} \operatorname{Tr}[\rho(\log \rho-\log \sigma)]
$$

as $\alpha \rightarrow 1$, i.e.

$$
D_{1}^{(t)}(\rho \| \sigma):=\lim _{\alpha \rightarrow 1} D_{\alpha}^{(t)}(\rho \| \sigma)=D(\rho \| \sigma) .
$$

The cases of $D_{0}^{(t)}$ and $D_{\infty}^{(t)}$ are defined as limits of $D_{\alpha}^{*}$ for $\alpha \rightarrow\{0, \infty\}$. The following Lemma 1 collects useful properties of the $\alpha$-Rényi divergence and ensures the existence of $D_{0}^{(t)}$ and $D_{\infty}^{(t)}$.

\footnotetext{
${ }^{8}$ The three quantities are finite when $\rho \ll \sigma$, or $\rho$ is not orthogonal to $\sigma$ and $\alpha<1$. Otherwise, they are positive infinite.
} 
Lemma 1 (Properties of order $\alpha$ Rényi Divergences). The following holds.

(a) Let $(t)=\{\}$,* or b. For any $\rho \in \mathcal{S}(\mathcal{H})$, the map $\alpha \rightarrow D_{\alpha}^{(t)}(\rho \| \sigma)$ is continuous and nondecreasing on $[0, \infty]$.

(b) Let $(t)=\{\}$,* or b. For $\rho, \sigma \in \mathcal{L}_{>0}(\mathcal{H})$, and $\alpha \in(0, \infty]$, we have $D_{\alpha}^{(t)}(\rho \| \sigma) \geq \log \operatorname{Tr}[\rho]-\log \operatorname{Tr}[\sigma]$ with equality if and only if $\rho$ is a constant multiple of $\sigma$. Moreover, $D_{\alpha}^{(t)}(\rho \| \sigma) \geq 0$ for $\rho, \sigma \in \mathcal{S}(\mathcal{H})$ with equality if and only if $\rho=\sigma$.

(c) For any $\rho, \sigma_{1}, \sigma_{2} \in \mathcal{L}_{>0}(\mathcal{H})$ with $\sigma_{1} \leq \sigma_{2}$, we have $D_{\alpha}^{(t)}\left(\rho \| \sigma_{1}\right) \geq D_{\alpha}^{(t)}\left(\rho \| \sigma_{2}\right)$ for $(t)=\{\}$ and $\alpha \in[0,1]$, for $(t)=*$ and $\alpha \in[1 / 2, \infty]$, and for $(t)=b$ and $\alpha \in[0, \infty]$.

(d) For any $\rho \in \mathcal{L}_{>0}(H), D_{\alpha}^{(t)}(\rho \| \sigma)$ is convex on $\mathcal{S}(\mathcal{H})$ for $(t)=\{\}$ and $\alpha \in[0,2]$, for $(t)=*$ and $\alpha \in[1 / 2, \infty]$. and for $(t)=b$ and $\alpha \in[0, \infty]$

(e) Let $(t)=\{\}$,* or b. For any $\alpha \in(0, \infty]$ and $\rho \in \mathcal{L}_{>0}(\mathcal{H}), \sigma \mapsto D_{\alpha}^{(t)}(\rho \| \sigma)$ is lower semi-continuous on $\mathcal{S}(\mathcal{H})$.

(f) For any $\rho, \sigma \in \mathcal{L}_{>0}(H)$, we have

$$
\begin{array}{ll}
D_{\alpha}^{*}(\rho \| \sigma) \leq D_{\alpha}(\rho \| \sigma) \leq D_{\alpha}^{b}(\rho \| \sigma), & \alpha \in[0,1], \\
D_{\alpha}^{b}(\rho \| \sigma) \leq D_{\alpha}^{*}(\rho \| \sigma) \leq D_{\alpha}(\rho \| \sigma), & \alpha \in[1, \infty] .
\end{array}
$$

We note that (a) was proved in [86, Lemma 3.12, Corollary 3.15] and [81, Theorem 7]; (b) was shown in [81, Theorem 3], [104, Theorem 5] and [86, Proposition 3.22]; (c) was proved in [81, Proposition 4] and [86, Lemma 3.24]; (d) was shown in [86, Proposition 3.18] $]^{9}$, (e) was proved in [29, Theorem 15], [86, Corollary 3.27]; and (f) is a consequence of the Araki-Lieb-Thirring inequality [105, 106] and GoldenThompson inequality [95, 96, 97] (see also [86, Proposition 3.20]).

Finally, for $\rho, \sigma \in \mathcal{S}(\mathcal{H})$, the quantum relative entropy variances [107, 108] are defined as

$$
\begin{aligned}
& V^{(t)}(\rho \| \sigma):=\operatorname{Tr}\left[\rho(\log \rho-\log \sigma)^{2}\right]-D(\rho \| \sigma)^{2}, \quad(t)=\{\} \text { or } * \\
& V^{b}(\rho \| \sigma):=\int_{0}^{1} \mathrm{~d} t \operatorname{Tr}\left[\rho^{1-t}(\log \rho-\log \sigma) \rho^{t}(\log \rho-\log \sigma)\right]-D(\rho \| \sigma)^{2} .
\end{aligned}
$$

\section{Complex Interpolation and Noncommutative $L_{p}$ Spaces}

In this section, we first recall the definition of the complex interpolation and the noncommutative $L_{p}$ spaces. The main result in this section is an interpolation inequality corresponding to a quasi-norm defined in Eq. (15). This will be employed to prove the concavity of Petz's auxiliary function in Section 5.

Let us start with complex interpolation. We refer to [109] for a detailed account of interpolation spaces. Let $X_{0}$ and $X_{1}$ be two Banach spaces. Assume that there exists a Hausdorff topological vector space $X$ such that $X_{0}, X_{1} \subset X$ as subspaces. Let $\mathcal{Z}=\{z \mid 0 \leq \operatorname{Re}(z) \leq 1\}$ be the unit vertical strip on the complex plane, and $\mathcal{Z}_{0}=\{z \mid 0<\operatorname{Re}(z)<1\}$ be its open interior. Let $\mathcal{F}\left(X_{0}, X_{1}\right)$ be the space of all functions $f: \mathcal{Z} \rightarrow X_{0}+X_{1}$, which are bounded and continuous on $\mathcal{Z}$ and analytic on $\mathcal{Z}_{0}$, and moreover

$$
\{f(\mathrm{i} t) \mid t \in \mathbb{R}\} \subset X_{0},\{f(1+\mathrm{i} t) \mid t \in \mathbb{R}\} \subset X_{1} .
$$

$\mathcal{F}\left(X_{0}, X_{1}\right)$ is again a Banach space equipped with the norm

$$
\|f\|_{\mathcal{F}}:=\max \left\{\sup _{t \in \mathbb{R}}\|f(\mathrm{i} t)\|_{X_{0}}, \sup _{t \in \mathbb{R}}\|f(1+\mathrm{i} t)\|_{X_{1}}\right\} .
$$

The complex interpolation space $\left(X_{0}, X_{1}\right)_{\theta}$, for $0 \leq \theta \leq 1$, is the quotient space of $\mathcal{F}\left(X_{0}, X_{1}\right)$ as follows,

$$
\left(X_{0}, X_{1}\right)_{\theta}=\left\{x \in X_{0}+X_{1} \mid x=f(\theta) \text { for some } f \in \mathcal{F}\left(X_{0}, X_{1}\right)\right\} \text {. }
$$

\footnotetext{
${ }^{9}$ We note that in Ref. [81] the definitions for the three types of Rényi divergences do not have the scaling factor Tr$[\rho]$ in the denominator. Here, we adopt the notation introduced in Ref. [86]. For [86, Proposition 3.18] (corresponding to Lemma 1-(d)) and [86, Corollary 3.27] (corresponding to Lemma 1-(e)), we remark that the proofs work for both definitions of the Rényi divergences.
} 
where quotient norm is

$$
\|x\|_{\theta}=\inf \left\{\|f\|_{\mathcal{F}} \mid f(\theta)=x\right\} .
$$

It is clear from the definition that $X_{0}=\left(X_{0}, X_{1}\right)_{0}, X_{1}=\left(X_{0}, X_{1}\right)_{1}$. For all $0<\theta<1,\left(X_{0}, X_{1}\right)_{\theta}$ are called interpolation space of $\left(X_{0}, X_{1}\right)$. A consequence of the definition (11) is the interpolation inequality that for an analytic function $f: \mathcal{Z} \rightarrow X_{0}+X_{1}$,

$$
\|f(\theta)\|_{\left(X_{0}, X_{1}\right)_{\theta}} \leq\left(\sup _{t \in \mathbb{R}}\|f(\mathrm{i} t)\|_{X_{0}}\right)^{1-\theta}\left(\sup _{t \in \mathbb{R}}\|f(1+\mathrm{i} t)\|_{X_{1}}\right)^{\theta} .
$$

This follows from applying the definition to the analytic function

$$
\tilde{f}(z)=\left(\sup _{t \in \mathbb{R}}\|f(\mathrm{i} t)\|_{X_{0}}\right)^{z-1}\left(\sup _{t \in \mathbb{R}}\|f(1+\mathrm{i} t)\|_{X_{1}}\right)^{-z} f(z) .
$$

The most basic example is that the $p$-integrable function spaces $L_{p}(\Omega, \mu)$ of a positive measure space $(\Omega, \mu)$. $L_{p}(\Omega, \mu)$ for $1 \leq p \leq \infty$ forms a family of interpolation spaces, i.e.

$$
L_{p}(\Omega, \mu) \cong\left[L_{p_{0}}(\Omega, \mu), L_{p_{1}}(\Omega, \mu)\right]_{\theta}
$$

holds isometrically for all $1 \leq p_{0}, p_{1}, p \leq \infty, 0 \leq \theta \leq 1$ such that $\frac{1}{p}=\frac{1-\theta}{p_{0}}+\frac{\theta}{p_{1}}$. For a von Neumann algebra $(\mathcal{M}, \mathrm{Tr})$ equipped with normal faithful semifinite trace $\mathrm{Tr}$, the noncommutative $L_{p}$-norm is defined as $\|x\|_{p}=\operatorname{Tr}\left(|x|^{p}\right)^{\frac{1}{p}}$ and $L_{p}(\mathcal{M}, \operatorname{Tr})$ (or shortly $\left.L_{p}(\mathcal{M})\right)$ is the completion of $\left\{x \in \mathcal{M} \mid\|x\|_{p}<\infty\right\}$. The analog of (12) is that

$$
L_{p}(\mathcal{M}, \operatorname{Tr}) \cong\left[L_{p_{0}}(\mathcal{M}, \operatorname{Tr}), L_{p_{1}}(\mathcal{M}, \operatorname{Tr})\right]_{\theta} .
$$

In particular, the Schatten- $p$ class on a Hilbert space $\mathcal{H}$ satisfies

$$
S_{p}(\mathcal{H}) \cong\left[S_{p_{0}}(\mathcal{H}), S_{p_{1}}(\mathcal{H})\right]_{\theta} .
$$

Here $S_{\infty}(\mathcal{H}):=\mathcal{B}(\mathcal{H})$ denotes the bounded operators on $\mathcal{H}$. The interpolation relation has already been used in many works in quantum information theory, e.g. [82].

In [99] Junge and Parcet introduced the amalgamated $L_{p}$-space for a subalgebra $\mathcal{N} \subset \mathcal{M}$. Here, for simplicity, we consider the case $(\mathcal{M}, \operatorname{Tr})$ being a semi-finite von Neumann algebra and $\mathcal{N}$ is a subalgebra of $\mathcal{M}$ such that $\operatorname{Tr}_{\mid \mathcal{N}}$ is also semi-finite. Let $1 \leq p \leq \infty$ and $\frac{1}{p}+\frac{1}{p^{\prime}}=1$. For $x \in \mathcal{M}$, the amalgamated $L_{1}^{p}$ norm of the inclusion $\mathcal{N} \subset \mathcal{M}$ is defined as

$$
\|x\|_{L_{1}^{p}(\mathcal{N} \subset \mathcal{M})}:=\inf \left\{\|a\|_{2 p^{\prime}}\|y\|_{p}\|b\|_{2 p^{\prime}} \mid x=a y b\right\},
$$

where the infimum is taken over all factorizations $x=a y b$ such that $a, b \in L_{2 p^{\prime}}(\mathcal{N})$ and $y \in L_{p}(\mathcal{M})$. When $x$ is positive, the above expression simplifies to

$$
\|x\|_{L_{1}^{p}(\mathcal{N} \subset \mathcal{M})}=\inf _{\sigma \in \mathcal{S}(\mathcal{N})}\left\|\sigma^{-\frac{1}{2 p^{r}}} x \sigma^{-\frac{1}{2 p^{r}}}\right\|_{p} .
$$

Here and in the following, the infimum is taken over all density operators $\sigma \in \mathcal{N}$ such that $x=\sigma^{\frac{1}{2 p^{\prime}}} y \sigma^{\frac{1}{2 p^{\prime}}}$ with $y \in L_{p}(\mathcal{M})$ so that $\sigma^{-\frac{1}{2 p^{\prime}}} x \sigma^{-\frac{1}{2 p}}$ is well defined. For example, let $\mathcal{N}=\mathbb{1}_{\mathcal{H}_{A}} \otimes \mathcal{B}\left(\mathcal{H}_{B}\right)$ and $\mathcal{M}=$ $\mathcal{B}\left(\mathcal{H}_{A}\right) \otimes \mathcal{B}\left(\mathcal{H}_{B}\right)$ equipped with the usual matrix trace on $\mathcal{H}_{A}$ and $\mathcal{H}_{A} \otimes \mathcal{H}_{B}$, respectively. This is the $L_{p}$-norm corresponding to sandwiched conditional Rényi entropy [81, 82, 78]:

$$
\begin{aligned}
H_{p}^{*}(A \mid B) & :=-\inf _{\sigma_{B} \in \mathcal{B}\left(\mathcal{H}_{B}\right)} D_{p}^{*}\left(\rho_{A B} \| \mathbb{1}_{\mathcal{H}_{A}} \otimes \sigma_{B}\right) \\
& =-p^{\prime} \log \left\|\rho^{A B}\right\|_{L_{1}^{p}\left(\mathbb{1}_{\mathcal{H}_{A}} \otimes \mathcal{B}\left(\mathcal{H}_{A}\right) \subset \mathcal{B}\left(\mathcal{H}_{A}\right) \otimes \mathcal{B}\left(\mathcal{H}_{B}\right)\right)} .
\end{aligned}
$$

This special case was introduced and studied by Pisier in [100] as vector-valued noncommutative $L_{p^{-}}$ spaces. In general, for an inclusion $\mathcal{N} \subset \mathcal{M}$, Junge and Parcet proved that

i) $\|\cdot\|_{L_{1}^{p}(\mathcal{N} \subset \mathcal{M})}$ is indeed a Banach space norm for $1 \leq p \leq \infty$. 
ii) for all $1 \leq p_{0}, p_{1}, p \leq \infty, 0 \leq \theta \leq 1$ such that $\frac{1}{p}=\frac{1-\theta}{p_{0}}+\frac{\theta}{p_{1}}$,

$$
\left.L_{1}^{p_{0}}(\mathcal{N} \subset \mathcal{M}), L_{1}^{p_{1}}(\mathcal{N} \subset \mathcal{M})\right]_{\theta} \cong L_{1}^{p}(\mathcal{N} \subset \mathcal{M})
$$

holds isometrically.

We also consider the expression corresponding to Petz's version. We define that for a positive $x$,

$$
\|x\|_{\bar{L}_{1}^{p}(\mathcal{N} \subset \mathcal{M})}:=\inf _{\sigma \in \mathcal{S}(\mathcal{N})}\left(\operatorname{Tr}\left[x^{p} \sigma^{1-p}\right]\right)^{\frac{1}{p}} .
$$

We have the following Sibson identity [27, 87] for a subalgebra $\mathcal{N} \subset \mathcal{M}$. Recall that the conditional expectation $\mathbb{E}: \mathcal{M} \rightarrow \mathcal{N}$ is a unique completely positive trace-preserving map such that

$$
\operatorname{Tr}(x a)=\operatorname{Tr}(\mathbb{E}(x) a), \mathbb{E}(a x b)=a \mathbb{E}(x) b, \forall a, b \in \mathcal{N}, x \in \mathcal{M} .
$$

Proposition 2 (Noncommutative Sibson Identity). Let $\mathcal{M}$ be a finite von Neumann algebra and $\mathcal{N} \subset \mathcal{M}$ be a subalgebra. For all $\rho \in \mathcal{S}(\mathcal{M}), \sigma \in \mathcal{S}(\mathcal{N})$, and $\alpha \in(0, \infty)$, it follows that

$$
\begin{aligned}
D_{\alpha}(\rho \| \sigma) & =D_{\alpha}\left(\sigma^{\star} \| \sigma\right)+\frac{\alpha}{\alpha-1} \log \operatorname{Tr}\left[\left(\mathbb{E}\left(\rho^{\alpha}\right)\right)^{\frac{1}{\alpha}}\right] \\
& =D_{\alpha}\left(\sigma^{\star} \| \sigma\right)+D_{\alpha}\left(\rho \| \sigma^{\star}\right),
\end{aligned}
$$

where

$$
\sigma^{\star}:=\frac{\left(\mathbb{E}\left(\rho^{\alpha}\right)\right)^{\frac{1}{\alpha}}}{\operatorname{Tr}\left[\left(\mathbb{E}\left(\rho^{\alpha}\right)\right)^{\frac{1}{\alpha}}\right]} .
$$

In particular, $\inf _{\sigma \in \mathcal{S}(\mathcal{N})} D_{\alpha}(\rho \| \sigma)=\frac{\alpha}{\alpha-1} \log \operatorname{Tr}\left[\left(\mathbb{E}\left(\rho^{\alpha}\right)\right)^{\frac{1}{\alpha}}\right]$

Proof. Using the property of conditional expectation,

$$
\begin{aligned}
D_{\alpha}(\rho \| \sigma) & =\frac{1}{\alpha-1} \log \operatorname{Tr}\left[\rho^{\alpha} \sigma^{1-\alpha}\right] \\
& =\frac{1}{\alpha-1} \log \operatorname{Tr}\left[\mathbb{E}\left(\rho^{\alpha}\right) \sigma^{1-\alpha}\right] \\
& =\frac{1}{\alpha-1} \log \operatorname{Tr}\left[\left(\sigma^{\star}\right)^{\alpha} \sigma^{1-\alpha}\right]+\frac{\alpha}{\alpha-1} \log \operatorname{Tr}\left(\mathbb{E}\left(\rho^{\alpha}\right)^{\frac{1}{\alpha}}\right) \\
& =D_{\alpha}\left(\sigma^{\star} \| \sigma\right)+\frac{\alpha}{\alpha-1} \log \operatorname{Tr}\left(\mathbb{E}\left(\rho^{\alpha}\right)^{\frac{1}{\alpha}}\right) .
\end{aligned}
$$

Note that

$$
\operatorname{Tr}\left(\mathbb{E}\left(\rho^{\alpha}\right)^{\frac{1}{\alpha}}\right)=\operatorname{Tr}\left(\mathbb{E}\left(\rho^{\alpha}\right) \mathbb{E}\left(\rho^{\alpha}\right)^{\frac{1-\alpha}{\alpha}}\right)=\operatorname{Tr}\left(\rho^{\alpha} \mathbb{E}\left(\rho^{\alpha}\right)^{\frac{1-\alpha}{\alpha}}\right)=\operatorname{Tr}\left(\rho^{\alpha}\left(\sigma^{\star}\right)^{1-\alpha}\right) \operatorname{Tr}\left(\mathbb{E}\left(\rho^{\alpha}\right)^{\frac{1}{\alpha}}\right)^{1-\alpha} .
$$

Thus

$$
\frac{\alpha}{\alpha-1} \log \operatorname{Tr}\left(\mathbb{E}\left(\rho^{\alpha}\right)^{\frac{1}{\alpha}}\right)=D_{\alpha}\left(\rho \| \sigma^{\star}\right) .
$$

The last assertion follows form the non-negativity of Petz's Rényi divergence $D_{\alpha}\left(\sigma^{\star} \| \sigma\right)$ (see e.g. [83]).

Remark 3.1. Proposition 2 is a generalization of the quantum Sibson identity proved by Sharma and Warsi [87] for the case $\mathcal{M}=\mathcal{B}\left(\mathcal{H}_{A} \otimes \mathcal{H}_{B}\right), \mathcal{N}=\mathbb{1}_{A} \otimes \mathcal{B}\left(\mathcal{H}_{B}\right)$ and $\mathbb{E}$ is the partial trace on system $A$. We observe that the quantum Sibson identity can be interpreted from a more general framework of noncommutative measure space with conditional expectation.

By using Proposition 2, we can rewrite for all $p \geq 1$,

$$
\|x\|_{\bar{L}_{1}^{p}(\mathcal{N} \subset \mathcal{M})}=\operatorname{Tr}\left[\mathbb{E}\left(x^{p}\right)^{\frac{1}{p}}\right] .
$$

For this quantity, we have the following interpolation type inequality. This might be of independent interest. 
Proposition 3 (The interpolation inequality for $\left.\|\cdot\|_{\bar{L}_{1}^{p}(\mathcal{N} \subset \mathcal{M})}\right)$. For every $x \in \mathcal{M}, 1<p_{0}, p_{1}, p<\infty$, $0 \leq \theta \leq 1$ such that $\frac{1}{p}=\frac{1-\theta}{p_{0}}+\frac{\theta}{p_{1}}$, it holds that

$$
\|x\|_{\bar{L}_{1}^{p}(\mathcal{N} \subset \mathcal{M})} \leq\|x\|_{\bar{L}_{1}^{p_{0}(\mathcal{N} \subset \mathcal{M})}}^{1-\theta}\|x\|_{\bar{L}_{1}^{p_{1}}(\mathcal{N} \subset \mathcal{M})}^{\theta^{p_{0}}} .
$$

Proof of Proposition 3. Denote $\gamma=\frac{p(1-\theta)}{p_{0}}$ and $1-\gamma=\frac{p \theta}{p_{1}}$. Let us first consider the case $\gamma=\frac{1}{2}$ and $p=\frac{1}{2}\left(p_{0}+p_{1}\right)$. In this case, the inequality, Eq. (16), is equivalent to that for any $\sigma_{0}, \sigma_{1} \in S(\mathcal{N})$,

$$
\left\|x^{\frac{p_{0}}{2}} \sigma_{0}^{\frac{1-p_{0}}{2}}\right\|_{2}\left\|x^{\frac{p_{1}}{2}} \sigma_{1}^{\frac{1-p_{1}}{2}}\right\|_{2} \geq\left\|\mathbb{E}\left(x^{p}\right)\right\|_{\frac{1}{p}} .
$$

Starting with Cauchy-Schwartz inequality,

$$
\begin{aligned}
\left\|x^{\frac{p_{0}}{2}} \sigma_{0}^{\frac{1-p_{0}}{2}}\right\|_{2}\left\|x^{\frac{p_{1}}{2}} \sigma_{1}^{\frac{1-p_{1}}{2}}\right\|_{2} & \geq\left\|\sigma_{1}^{\frac{1-p_{1}}{2}} x^{\frac{p_{1}}{2}} x^{\frac{p_{0}}{2}} \sigma_{0}^{\frac{1-p_{0}}{2}}\right\|_{1} \\
& =\left\|\sigma_{1}^{\frac{1-p_{1}}{2}} x^{p} \sigma_{0}^{\frac{1-p_{0}}{2}}\right\|_{1} \\
& \geq\left\|\mathbb{E}\left(\sigma_{1}^{\frac{1-p_{1}}{2}} x^{p} \sigma_{0}^{\frac{1-p_{0}}{2}}\right)\right\|_{1} \\
& =\left\|\sigma_{1}^{\frac{1-p_{1}}{2}} \mathbb{E}\left(x^{p}\right) \sigma_{0}^{\frac{1-p_{0}}{2}}\right\|_{1} \\
& \geq\left\|\mathbb{E}\left(x^{p}\right)\right\|_{\frac{1}{p}},
\end{aligned}
$$

where inequality (17) follows from the fact that the conditional expectation $\mathbb{E}$ is a contraction for 1-norm, and Eq. (18) uses the module property of $\mathbb{E}$, i.e.

$$
\mathbb{E}(a x b)=a \mathbb{E}(x) b, \quad \forall a, b \in \mathcal{N}, x \in \mathcal{M} .
$$

The last inequality (19) is the Hölder inequality for $p=1+\frac{p_{0}-1}{2}+\frac{p_{1}-1}{2}$,

$$
\begin{aligned}
\left\|\mathbb{E}\left(x^{p}\right)\right\|_{\frac{1}{p}} & \leq\left\|\sigma_{1}^{\frac{p_{1}-1}{2}}\right\|_{\frac{2}{p_{1}-1}}\left\|\sigma_{1}^{\frac{1-p_{1}}{2}} \mathbb{E}\left(x^{p}\right) \sigma_{0}^{\frac{1-p_{0}}{2}}\right\|_{1}\left\|\sigma_{0}^{\frac{p_{0}-1}{2}}\right\|_{\frac{2}{p_{0}-1}} \\
& \leq\left\|\sigma_{1}^{\frac{1-p_{1}}{2}} \mathbb{E}\left(x^{p}\right) \sigma_{0}^{\frac{1-p_{0}}{2}}\right\|_{1} .
\end{aligned}
$$

Here, $\left\|\sigma_{1}\right\|_{1}=\left\|\sigma_{0}\right\|_{1}=1$ because they are density operators.

This proves the inequality for $\gamma=\frac{1}{2}$. Using induction, we obtain the inequality for $2^{n}$-partition points $p=k 2^{-n}\left(p_{1}-p_{0}\right)+p_{0}$ for all $k, n \in \mathbb{N}, 0 \leq k \leq 2^{n}$. The case for general $p$ follows from the continuity.

Remark 3.2. For finite-dimensional matrices, the above interpolation inequality, Eq. (16), is a special case of [110, Corollary 3.7] with trace norm: for all unitary-invariant norms $\|\cdot\| \mid \|$ and $\nu>0$, the map

$$
(p, t) \mapsto\left\|\left(\Lambda\left(A^{\frac{t}{p}}\right)\right)^{\nu p}\right\|
$$

is jointly log-convex on $(0, \infty) \times(-\infty, \infty)$ for any positive linear maps $\Lambda$ on positive semi-definite matrices. We remark that for von Neumann algebras similar results have been studied by Shao [111]. In particular, our inequality for conditional expectation can also be derived from [111, Corollary 3.13] via an averaging trick.

\section{Noncommutative Rényi and Augustin Information}

In this section, we introduce the Rényi information and Augustin information. These quantities are usually defined for a channel. However, in the case of classical-quantum channels, the channel output can be viewed as a collection of density operators or noncommutative measures. We can thus define the Rényi and Augustin information from a perspective of noncommutative measure space without introducing the classical-quantum channels. The connection can be easily understood, and we delay this until Section 6 . 
We firstly establish fundamental properties for both the Rényi and Augustin information (Propositions 4 and 5). Secondly, in Section 4.1 we study whether the infimum in the definition of the Rényi information can be attained.

Let $\mathcal{W} \subset \mathcal{S}(\mathcal{H})$ be a set of density operators on $\mathcal{H}$. Given a prior probability mass function $P \in$ $\mathcal{P}(\mathcal{W})$, and $\alpha \in[0, \infty]$, we define the order $\alpha$ Rényi information and the order $\alpha$ Augustin information, respectively, by

$$
\begin{aligned}
I_{\alpha}^{\mathrm{r},(t)}(P, \mathcal{W}) & :=\inf _{\sigma \in \mathcal{S}(\mathcal{H})} D_{\alpha}^{(t)}(P \circ \mathcal{W} \| P \otimes \sigma), \\
I_{\alpha}^{\mathrm{a},(t)}(P, \mathcal{W}) & :=\inf _{\sigma \in \mathcal{S}(\mathcal{H})} D_{\alpha}^{(t)}(\omega \| \sigma \mid P):=\inf _{\sigma \in \mathcal{S}(\mathcal{H})} \sum_{\omega \in \mathcal{W}} P(\omega) D_{\alpha}^{(t)}(\omega \| \sigma) .
\end{aligned}
$$

for $(t)=\{\},\{*\}$ and $\{b\}$. Here, $P \circ \mathcal{W}:=\sum_{\omega} P(\omega)|\omega\rangle\langle\omega| \otimes \omega$ is a joint probability measure whose marginal distribution on the support of $P$ is $P$ and whose conditional distribution is $\omega \in \mathcal{W}$. Here, we use the superscript ' $r$ ' to indicate the Rényi information, while ' $a$ ' to indicate the Augustin information. As we defined for the Rényi divergence, the superscript ' $(t)$ ' denotes that the information quantities are defined by the Petz $(t)=\{\}$, sandwiched $(t)=\{*\}$, or the log-Euclidean form $(t)=\{b\}$.

For $\alpha=1$, these two quantities correspond to the Holevo quantity [112]:

$$
I_{1}^{\mathrm{r},(t)}(P, \mathcal{W})=I_{1}^{\mathrm{a},(t)}(P, \mathcal{W})=I(P, \mathcal{W}):=D(P \circ \mathcal{W} \| P \otimes P \mathcal{W}),
$$

where $P \mathcal{W}:=\sum_{w \in \mathcal{W}} P(\omega) \omega$ is the marginal state on the output Hilbert space. If the measures are commutative, it is exactly Shannon's mutual information.

The order- $\alpha$ Rényi capacity is defined as follows [86, Proposition 4.2, Corolary 4.5]:

$$
\begin{aligned}
C_{\alpha, \mathcal{W}}^{(t)} & :=\sup _{P \in \mathcal{P}(\mathcal{W})} I_{\alpha}^{\mathrm{r},(t)}(P, \mathcal{W}) \\
& =\sup _{P \in \mathcal{P}(\mathcal{W})} I_{\alpha}^{\mathrm{a},(t)}(P, \mathcal{W})
\end{aligned}
$$

for $(t)=\{\}$ and $\alpha \in[0,2]$, for $(t)=*$ and $\alpha \in[1 / 2, \infty]$, and for $(t)=b$ and $\alpha \in[0, \infty]$.

In the following Propositions 4 and 5, we prove important properties of the Rényi and Augustin information.

Proposition 4 (Properties of Rényi Information). Let $\mathcal{W} \subset \mathcal{S}(\mathcal{H})$, and let $(t)$ be any of the three values: \{\}$, *$ or $b$.

(a) For any $P \in \mathcal{P}(\mathcal{W}), I_{\alpha}^{\mathrm{r},(t)}(P, \mathcal{W})$ is non-negative and nondecreasing in $\alpha$. Moreover, $I_{\alpha}^{\mathrm{r},(t)}(P, \mathcal{W}) \leq$ $\log |\operatorname{supp}(P)|$.

(b) The map $P \mapsto I_{\alpha}^{\mathrm{r},(t)}(P, \mathcal{W})$ is quasi-concave on $P \in \mathcal{P}(\mathcal{W})$ for $\alpha \in[0,1)$, and concave on $P \in \mathcal{P}(\mathcal{W})$ for $\alpha \in[1, \infty]$.

(c) Let

$$
\mathcal{A}:=[0,1], \quad \mathcal{A}^{*}:=[1 / 2, \infty], \text { and } \mathcal{A}^{b}:=[0, \infty] .
$$

For $\eta \in[0, \infty]$, if $C_{\eta, \mathcal{W}}^{(t)}<\infty$, then $\left\{I_{\alpha}^{\mathrm{r},(t)}(P, \mathcal{W})\right\}_{\alpha \in[0, \eta] \cap \mathcal{A}^{(t)}}$ is uniformly equicontinuous in $P \in$ $\mathcal{P}(\mathcal{W})$.

The proof of Proposition 4 is given in Appendix A.

Proposition 5 (Properties of Augustin Information). Let $\mathcal{W} \subset \mathcal{S}(\mathcal{H})$ be a classical-quantum channel, $(t)$ be any of the three values: \{\}$, *$, or $b$, and let $\mathcal{A}^{(t)}$ be defined in (23).

(a) For every $P \in \mathcal{P}(\mathcal{X}), I_{\alpha}^{\mathrm{a},(t)}(P, \mathcal{W})$ is non-negative and nondecreasing in $\alpha$. Moreover, $I_{\alpha}^{\mathrm{a},(t)}(P, \mathcal{W}) \leq$ $H(P)$ for $\alpha \in \mathcal{A}^{(t)}$, where $H(P)$ is the Shannon entropy of $P$.

(b) For any $\alpha>0$, the map $P \mapsto I_{\alpha}^{\mathrm{a},(t)}(P, \mathcal{W})$ is concave on $P \in \mathcal{P}(\mathcal{W})$.

(c) For $\eta \in[0, \infty]$, if $C_{\eta, \mathcal{W}}^{(t)}<\infty$, then $\left\{I_{\alpha}^{\mathrm{a},(t)}(P, \mathcal{W})\right\}_{\alpha \in(0, \eta] \cap \mathcal{A}^{(t)}}$ is uniformly equicontinuous in $P \in$ $\mathcal{P}(\mathcal{W})$. 
The proof of Proposition 5 is given in Appendix A.

4.1. Existence of Infimum. The noncommutative Sibson identity established in Proposition 2 already guarantees that the infimum of $I_{\alpha}^{r}$ in Eq. (20) is attained for all $\alpha \in(0, \infty)$. The goal of this section is to show that the infimum of $I_{\alpha}^{\mathrm{r}, *}$ is attained for all $\alpha \in[1, \infty]$ (Corollary 9).

We have already noted that the sandwiched Rényi information $I_{\alpha}^{\mathrm{r}, *}(P, \mathcal{W})$ is closely related to the noncommutative $L_{1}^{\alpha}$ space as follows,

$$
I_{\alpha}^{\mathrm{r}, *}(P, \mathcal{W})=\alpha^{\prime} \log \|\rho\|_{S_{1}\left(\mathcal{H}, l_{\alpha}^{n}\right)}
$$

where $\rho=\oplus_{\omega} P(\omega)^{\frac{1}{\alpha}} \omega, l_{\alpha}^{n}$ is the $\alpha$-summable space for length $n$-sequence and in our case $\mathcal{H}$ is the separable infinite-dimensional Hilbert space. This is a special case of vector-valued $L_{p}$ space introduced by Pisier [100]. Recall that for a general element $x \in S_{1}\left(\mathcal{H}, l_{\alpha}^{n}\right)$, the norm is defined as

$$
\begin{aligned}
\|x\|_{S_{1}\left(\mathcal{H}, l_{\alpha}^{n}\right)} & =\inf _{x=(a \otimes \mathbb{1}) y(b \otimes \mathbb{1})}\|a\|_{S_{2 \alpha^{\prime}}}\|y\|_{S_{\alpha}\left(\mathcal{H}, l_{\alpha}^{n}\right)}\|b\|_{S_{2 \alpha^{\prime}}} \\
& =\inf _{x=(a \otimes \mathbb{1}) y(b \otimes \mathbb{1})}\|a\|_{S_{2 \alpha^{\prime}}}\|y\|_{L_{\alpha}\left(\oplus_{i=1}^{n} \mathcal{B}(\mathcal{H})\right)}\|b\|_{S_{2 \alpha^{\prime}}},
\end{aligned}
$$

where the infimum takes all factorization $x=(a \otimes \mathbb{1}) y(b \otimes \mathbb{1})$ with $a, b \in S_{2 \alpha^{\prime}}$. For positive $x$, it suffices to consider $a=b \geq 0$, and then the norm can be rewritten as

$$
\|x\|_{S_{1}\left(\mathcal{H}, l_{\alpha}^{n}\right)}=\inf _{\sigma \in \mathcal{S}(\mathcal{H})}\left\|\left(\sigma^{-\frac{1}{2 \alpha^{\prime}}} \otimes \mathbb{1}\right) x\left(\sigma^{-\frac{1}{2 \alpha^{\prime}}} \otimes \mathbb{1}\right)\right\|_{\alpha},
$$

which links to the sandwiched Rényi information $I_{\alpha}^{\mathrm{r}, *}$.

For a general von Neumann algebra $\mathcal{M}$, the space $L_{p}\left(\mathcal{M}, l_{\infty}^{n}\right)$ has been studied in [113, 114] for the purpose to understand the noncommutative martingale and maximal functions. In particular, [113, Remark 3.7] states that for a positive $x$, the above infimum for $\alpha=\infty$ can be attained, which follows from a Grothendieck-Pietsch factorization theorem. Namely, for $\rho=\oplus_{\omega} p(\omega)^{\frac{1}{\alpha}} \omega \in S_{1}\left(\mathcal{H}, l_{1}^{n}\right)$, there exists a density $\sigma^{\star} \in \mathcal{S}(\mathcal{H})$ such that

$$
\|\rho\|_{S_{1}\left(\mathcal{H}, l_{\alpha}^{n}\right)}=\inf _{\sigma \in \mathcal{S}(\mathcal{H})}\left\|\left(\sigma^{-\frac{1}{2}} \otimes \mathbb{1}\right) \rho\left(\sigma^{-\frac{1}{2}} \otimes \mathbb{1}\right)\right\|_{\infty}=\sum_{\omega} P(\omega)\left\|\left(\sigma^{\star}\right)^{-\frac{1}{2}} \omega\left(\sigma^{\star}\right)^{-\frac{1}{2}}\right\|_{\infty},
$$

which indicates the infimum in the definition of $I_{\infty}^{\mathrm{r}, *}$ is attained. Here, we show that such infimums are also attained for $1<\alpha<\infty$. Our argument uses the uniform convexity of noncommutative $L_{p}$ space. Here let us start with recalling the duality relation that for $1 \leq p<\infty$ and $p^{\prime}$ satisfying $\frac{1}{p}+\frac{1}{p^{\prime}}=1$,

$$
L_{p}(\mathcal{M})^{*}=L_{p^{\prime}}(\mathcal{M}) .
$$

In particular, this implies that for $1<p<\infty, L_{p}(\mathcal{M})^{* *}=L_{p}(\mathcal{M})$ are reflective. A stronger property for Banach spaces is the uniform convexity.

Definition 6. A normed space $X$ is uniform convex if for any $0<\epsilon<2$, there exists a $\delta>0$ such that if $\|x\|=\|y\|=1$ and $\left\|\frac{x+y}{2}\right\| \geq 1-\delta$, then $\|x-y\| \leq \epsilon$.

For $1<p<\infty$, the uniform convexity of noncommutative $L_{p}(\mathcal{M})$ follows from the Clarkson type inequality (see $[115,116,117]$, it is also clear that $L_{1}(\mathcal{M})$ and $L_{\infty}(\mathcal{M})$ are not uniform convex) We will use the following consequence of uniform convexity.

Lemma 7. Let $1<p<\infty$ and $\left(x_{n}\right)_{n \in \mathbb{N}}$ be an infinite sequence of positive element in $L_{2 p}(\mathcal{M})$. Suppose

(a) $\left\|x_{n}^{2}\right\|_{2 p}=1$ for all $n \in \mathbb{N}$.

(b) $\lim _{N \rightarrow \infty} \inf _{n, m>N}\left\|\frac{x_{n}^{2}+x_{m}^{2}}{2}\right\|_{p}=1$.

Then $\left(x_{n}\right)$ is a Cauchy sequence and hence $\lim _{n \rightarrow \infty} x_{n}=x \in L_{2 p}(\mathcal{M})$ for some $x$ with $\|x\|_{2 p}=1$. 
Proof. Given $\epsilon>0$, we choose $\delta>0$ such that if $\|a\|_{p}=\|b\|_{p}=1$ and $\left\|\frac{a+b}{2}\right\|_{p} \geq 1-\delta$, then $\|a-b\|_{p} \leq \epsilon$. By assumption, there exists $N$ large enough such that for all $n, m \geq N$,

$$
\left\|\frac{x_{n}^{2}+x_{m}^{2}}{2}\right\|_{p} \geq 1-\delta,
$$

which implies

$$
\left\|x_{n}^{2}-x_{m}^{2}\right\|_{p} \leq \epsilon
$$

Then the assertion follows from the inequality [118, Lemma 2.1]

$$
\left\|x_{n}-y_{m}\right\|_{2 p} \leq\left\|x_{n}^{2}-y_{m}^{2}\right\|_{p}^{\frac{1}{2}} \leq \epsilon .
$$

To show that the infimum in the definition of sandwiched Rényi information $I_{\alpha}^{r, *}$ is attained, it is sufficient to consider that for any positive $x$, the factorization norm

$$
\begin{aligned}
\|x\|_{S_{1}\left(l_{\alpha}\right)} & =\inf _{x=(a \otimes 1) y(b \otimes 1)}\|a\|_{S_{2 \alpha^{\prime}}}\|y\|_{\alpha}\|b\|_{S_{2 \alpha^{\prime}}} \\
& =\inf _{x=(a \otimes \mathbb{1}) y(a \otimes \mathbb{1})}\|a\|_{S_{2 \alpha^{\prime}}}^{2}\|y\|_{\alpha} \\
& =\inf _{\sqrt{x}=(a \otimes \mathbb{1}) \eta}\|a\|_{S_{2 \alpha^{\prime}}}^{2}\|\eta\|_{2 \alpha}^{2}
\end{aligned}
$$

is attained indeed by a factorization $x=(a \otimes \mathbb{1}) y(a \otimes \mathbb{1})=(a \otimes \mathbb{1}) \eta \eta^{*}(a \otimes \mathbb{1})$.

Proposition 8. For $1<p<\infty$ and $x$ positive, the above infimum is attained, i.e. there exists a $\in S_{2 p^{\prime}}$ and $y \in S_{p}\left(l_{p}\right)$ such that $x=(a \otimes \mathbb{1}) y(a \otimes 1)$ and

$$
\|x\|_{S_{1}\left(l_{p}\right)}=\|a\|_{2 p^{\prime}}^{2}\|y\|_{S_{p}\left(l_{p}\right)} .
$$

Proof. Given $\|x\|_{S_{1}\left(l_{p}\right)}=1$, we have

$$
\inf _{\sqrt{x}=(a \otimes 1) \eta}\|a\|_{S_{2 p^{\prime}}}\|\eta\|_{2 p}=1 .
$$

We can find sequences $\left(a_{n}\right) \subset S_{2 p^{\prime}}$ and $\left(\eta_{n}\right) \subset S_{p}\left(l_{p}\right)$ such that for each $n, \sqrt{x}=\left(a_{n} \otimes 1\right) \eta_{n},\left\|a_{n}\right\|_{2 p^{\prime}}=1$ and

$$
\left\|\eta_{n}\right\|_{S_{2 p}} \geq 1, \lim _{n \rightarrow \infty}\left\|\eta_{n}\right\|_{S_{2 p}}=1
$$

Without loss of generality, we can assume $a_{n} \geq 0$. Consider the $2 \times 2$ factorization

$$
\left[\begin{array}{cc}
\sqrt{x} & 0 \\
0 & 0
\end{array}\right]=\left[\begin{array}{cc}
\frac{a_{n}}{\sqrt{2}} & \frac{a_{m}}{\sqrt{2}} \\
0 & 0
\end{array}\right] \cdot\left[\begin{array}{cc}
\frac{\eta_{n}}{\sqrt{2}} & 0 \\
\frac{\eta_{m}}{\sqrt{2}} & 0
\end{array}\right]
$$

Note that

$$
\begin{aligned}
& \left\|\left[\begin{array}{cc}
\frac{a_{n}}{\sqrt{2}} & \frac{a_{m}}{\sqrt{2}} \\
0 & 0
\end{array}\right]\right\|_{2 p^{\prime}}=\left\|\frac{a_{n}^{2}+a_{m}^{2}}{2}\right\|_{p^{\prime}}^{\frac{1}{2}} \\
& \left\|\left[\begin{array}{cc}
\frac{\eta_{n}}{\sqrt{2}} & 0 \\
\frac{\eta_{m}}{\sqrt{2}} & 0
\end{array}\right]\right\|_{2 p}=\left\|\frac{\eta_{n}^{*} \eta_{n}+\eta_{m}^{*} \eta_{m}}{2}\right\|_{p}^{\frac{1}{2}}
\end{aligned}
$$

Because

we know

$$
\left\|\left[\begin{array}{ll}
x & 0 \\
0 & 0
\end{array}\right]\right\|_{S_{1}\left(l_{p}\right)}=\|x\|_{S_{1}\left(l_{p}\right)}=1,
$$

$$
\left\|\left[\begin{array}{cc}
\frac{a_{n}}{\sqrt{2}} & \frac{a_{m}}{\sqrt{2}} \\
0 & 0
\end{array}\right]\right\|_{2 p^{\prime}}\left\|\left[\begin{array}{cc}
\frac{\eta_{n}}{\sqrt{2}} & 0 \\
\frac{\eta_{m}}{\sqrt{2}} & 0
\end{array}\right]\right\|_{2 p} \geq 1
$$


Moreover, since

$$
\left\|\frac{\eta_{n}^{*} \eta_{n}+\eta_{m}^{*} \eta_{m}}{2}\right\|_{p}^{\frac{1}{2}} \leq \frac{1}{2}\left\|\eta_{n}\right\|_{2 p}^{2}+\frac{1}{2}\left\|\eta_{m}\right\|_{2 p}^{2} \rightarrow 1 .
$$

we have

$$
\lim _{N \rightarrow \infty} \sup _{n, m \geq N} \|\left.\left[\begin{array}{cc}
\frac{\eta_{n}}{\sqrt{2}} & 0 \\
\frac{\eta_{m}}{\sqrt{2}} & 0
\end{array}\right]\right|_{2 p} \leq 1
$$

and hence

$$
\lim _{N \rightarrow \infty} \inf _{n, m \geq N}\left\|\frac{a_{n}^{2}+a_{m}^{2}}{2}\right\|_{p^{\prime}} \geq 1
$$

By Lemma 7, we know $a_{n} \rightarrow a$ in $S_{2 p^{\prime}}$ with $\|a\|_{2 p}=1$. On the other hand, because $S_{2 p}\left(l_{2 p}\right)$ is reflexive, there exists a subsequence $\eta_{n_{k}} \rightarrow y$ weakly in the unit ball of $S_{2 p}\left(l_{2 p}\right)$. Therefore, $\sqrt{x}=\left(a_{n_{k}} \otimes 1\right) \eta_{n_{k}} \rightarrow$ $(a \otimes 1) \eta$ weakly and hence $\sqrt{x}=(a \otimes 1) \eta$ and $\|\eta\|_{2 p}=1$. That completes the proof.

Corollary 9. For each $\mathcal{W} \subset \mathcal{S}(\mathcal{H})$ and $P \in \mathcal{P}(\mathcal{W})$, the infimum for $I_{\alpha}^{r, *}(P, \mathcal{W})$ is uniquely attained for $1 \leq \alpha \leq \infty$.

Proof. For $\alpha=1$, the infimum is attained by the (unnormalized) conditional expectation onto $\mathcal{B}(\mathcal{H})$,

$$
\mathbb{E}\left(\oplus_{\omega} p(\omega) \omega\right)=\sum_{\omega} p(\omega) \omega
$$

For $\alpha=\infty$, it is discussed as above. For $1<\alpha<\infty$, it is equivalent to show that for some $\gamma \in S(\mathcal{H})$

$$
\begin{aligned}
\|\rho\|_{S_{1}\left(\mathcal{H}, l_{\alpha}^{n}\right)} & =\left\|\left(\gamma^{-\frac{1}{2 \alpha^{\prime}}} \otimes 1\right) \rho\left(\gamma^{-\frac{1}{2 \alpha^{\prime}}} \otimes 1\right)\right\|_{\alpha} \\
& =\left\|\left(\gamma^{-\frac{1}{2 \alpha^{\prime}}} \otimes 1\right) \sqrt{\rho}\right\|_{2 \alpha}^{2},
\end{aligned}
$$

which follows from Proposition 8. The uniqueness is also guaranteed by the uniform convexity in Proposition 8 .

Remark 4.1. Although we only focus on the sandwiched Rényi information in Corollary 9, our approach given in Proposition 8 applies to the sandwiched conditional Rényi entropy. Namely, the infimum in (13) is uniquely attained for $1 \leq p \leq \infty$.

\section{Properties of Auxiliary Functions}

This section is devoted to proving fundamental properties of the auxiliary functions, which are defined as a scaled version of the Rényi information and Augustin information introduced in Section 4. Firstly, we present the convexity and concavity property of the auxiliary functions in prior probability distributions (Theorem 10). Secondly, we prove the concavity property in order $s \in(-1,0)$ for the sandwiched form (Theorem 11) and for the Petz form (Theorem 12), respectively, which thus answers the open problems as we described in Section 1. Lastly, we employ the concavity and the equicontinuity of the Rényi and Augustin information established in Section 4 to prove the joint continuity of the auxiliary functions (Theorem 13). We note that results established in this section can be extended to tracial von Neumann algebras (see Remark 5.2). We present our proof in terms of bounded operator $\mathcal{B}(\mathcal{H})$ since the usual quantum information-theoretic protocols are formulated accordingly (see Section 6).

Let $\mathcal{W} \subset \mathcal{S}(\mathcal{H})$ be an arbitrary set of density operators on $\mathcal{H}$. Given $s>-1$, and a prior probability mass function $P \in \mathcal{P}(\mathcal{W})$, and $(t) \in\{\}, *$, and $b$, we define the auxiliary functions for $\mathcal{W}$ in terms of the Rényi information and Augustin information:

$$
\begin{aligned}
& E_{0}^{\mathrm{r},(t)}(s, P):=s I_{\frac{1}{1+s}}^{\mathrm{r},(t)}(P, \mathcal{W}) \\
& E_{0}^{\mathrm{a},(t)}(s, P):=s I_{\frac{1}{1+s}}^{\mathrm{a},(t)}(P, \mathcal{W}) .
\end{aligned}
$$

As in Section 4, we use superscript $r$ to denote the Rényi information, whereas we use a to indicate the Augustin information. 
Theorem 10 (Convexity/Concavity in Prior). Let $\mathcal{W} \subset \mathcal{S}(\mathcal{H})$, and let $(t)$ be any of the three values. Then,

(a) $E_{0}^{r,(t)}(s, P)$ is quasi-concave in $P$ for $s \geq 0$, and convex in $P$ for $s \in[-1,0)$.

(b) $E_{0}^{\mathrm{a},(t)}(s, P)$ is concave in $P$ for $s \geq 0$, and convex in $P$ for $s \in[-1,0)$.

Proof of Theorem 10. The assertions follow from item (b) in Proposition 4, item (b) in Proposition 5, and the definitions given in Eq. (24) and (25).

In the following Theorem 11, we establish the concavity of the sandwiched auxiliary functions $E_{0}^{\mathrm{r}, *}(s, P)$ and $E_{0}^{r, *}(s, P)$.

Theorem 11 (Concavity of the Sandwiched Form in Order). Let $\mathcal{W} \subset \mathcal{S}(\mathcal{H})$ and $P \in \mathcal{P}(\mathcal{W})$ be a probability mass function.

(a) The map $s \mapsto E_{0}^{r, *}(s, P)$ is concave on $(-1,0)$.

(b) If $\mathcal{H}$ is finite-dimensional, then $s \mapsto E_{0}^{\mathrm{a}, *}(s, P)$ is concave on $(-1,0)$.

Before going into the details of the proof, we briefly sketch our proof strategy. To show the concavity of $E_{0}^{r, *}$, we introduce an analytic family of operators such that its amalgamated noncommutative norm coincides with the sandwiched Rényi information $I_{\alpha}^{\mathrm{r}, *}$. Then, the desired concavity follows from two facts: (i) the amalgamated noncommutative norm spaces form an interpolation family as we described in Section 3, and (ii) the amalgamated noncommutative norms of the analytic family of operators are bounded at the boundary of the complex strip.

Regarding the $E_{0}^{\mathrm{a}, *}$, we are not allowed to apply the interpolation inequality with respect to amalgamated noncommutative norms because the set of tensor power of operators is not a subalgebra. Instead, we directly prove an interpolation inequality for the target quantity by employing a technique of Devinatz's factorization theorem [119].

Remark 5.1. In Ref. [107, Corollary 10], Tomamichel and Hayashi proved a concavity of the map $(\alpha-$ 1) $\mapsto(1-\alpha) I_{\alpha}^{r, *}(P, \mathcal{W})$, which is slightly different from the auxiliary function considered in Theorem 11(a), namely, $\left.\alpha \mapsto E_{0}^{r, *}(s, P)\right|_{s=1 /(1+\alpha)}=\frac{1-\alpha}{\alpha} I_{\alpha}^{r, *}(P, \mathcal{W})$. Their proof technique is the so-called pinching argument, which allows the sandwiched Rényi information $I_{\alpha}^{r, *}$ to inherit properties from the commuting case. Nevertheless, such the approach relies on the finite-dimensional assumption, whereas our method in Theorem 11-(a) applies to infinite-dimensional Hilbert spaces as well as semi finite von Neumann algebras.

Proof of Theorem 11. We first prove the concavity for $E_{0}^{\mathrm{r}, *}(s, P)$ and then $E_{0}^{\mathrm{a}, *}(s, P)$. Note that the sandwiched Rényi information can be written as:

$$
\begin{aligned}
I_{\alpha}^{r, *}(P, \mathcal{W}) & =\inf _{\sigma \in \mathcal{S}(\mathcal{H})} D_{\alpha}^{*}\left(\oplus_{\omega} P(\omega) \omega \| \oplus_{\omega} P(\omega) \sigma\right) \\
& =\inf _{\sigma \in \mathcal{S}(\mathcal{H})} \frac{\alpha}{\alpha-1} \log \left\|\bigoplus_{\omega}(P(\omega) \sigma)^{\frac{1-\alpha}{2 \alpha}}(P(\omega) \omega)(P(\omega) \sigma)^{\frac{1-\alpha}{2 \alpha}}\right\|_{\alpha} \\
& =\inf _{\sigma \in \mathcal{S}(\mathcal{H})} \frac{\alpha}{\alpha-1} \log \left\|\bigoplus_{\omega} P^{\frac{1}{\alpha}}(\omega) \sigma^{\frac{1-\alpha}{2 \alpha}} \omega \sigma^{\frac{1-\alpha}{2 \alpha}}\right\|_{\alpha} \\
& =\inf _{\sigma \in \mathcal{S}(\mathcal{H})} \frac{\alpha}{\alpha-1} \log \left(\sum_{\omega} P(\omega)\left\|\sigma^{\frac{1-\alpha}{2 \alpha}} \omega \sigma^{\frac{1-\alpha}{2 \alpha}}\right\|_{\alpha}^{\alpha}\right)^{\frac{1}{\alpha}} .
\end{aligned}
$$

Using the substitution $\left.E_{0}^{r, *}(s, P)\right|_{s=(1-\alpha) / \alpha}=\frac{1-\alpha}{\alpha} I_{\alpha}^{r, *}(P, \mathcal{W})$, the concavity of $s \mapsto E_{0}^{r, *}(s, P)$ for $s \in$ $(-1,0)$, is equivalent to the log-convexity of the map:

$$
\frac{1}{\alpha} \mapsto \inf _{\sigma \in \mathcal{S}(\mathcal{H})}\left(\sum_{\omega} P(\omega)\left\|\sigma^{\frac{1-\alpha}{2 \alpha}} \omega \sigma^{\frac{1-\alpha}{2 \alpha}}\right\|_{\alpha}^{\alpha}\right)^{\frac{1}{\alpha}}, \quad \alpha>1 .
$$


To prove this, we let $\mathcal{X}:=\operatorname{supp}(P), \mathcal{M}=\oplus_{x \in \mathcal{X}} \mathcal{B}(\mathcal{H})$ and

$$
\mathcal{N}=1_{\mathcal{X}} \otimes \mathcal{B}(\mathcal{H}):=\{a \oplus a \oplus \cdots \oplus a \mid a \in \mathcal{B}(\mathcal{H})\} \subset \mathcal{M},
$$

and let $\frac{1}{\alpha}=\frac{1-\theta}{\alpha_{0}}+\frac{\theta}{\alpha_{1}}$ for $\alpha_{0}, \alpha_{1} \geq 1$ and $\theta \in[0,1]$. Define the analytic family of operators,

$$
\rho(z):=\bigoplus_{\omega \in \mathcal{W}} P(\omega)^{\frac{1-z}{\alpha_{0}}+\frac{z}{\alpha_{1}}} \cdot \omega \in \mathcal{M}, \quad \forall z \in \mathbb{C} .
$$

Note that $\rho(\theta)=\oplus_{\omega} P(\omega)^{\frac{1}{\alpha}} \omega$ and

$$
\begin{aligned}
\|\rho(\theta)\|_{L_{1}^{\alpha}(\mathcal{N} \subset \mathcal{M})} & :=n^{\frac{1}{\alpha^{\prime}}} \inf _{\sigma \in \mathcal{S}(\mathcal{H})}\left\|\left(\sigma^{\oplus n}\right)^{-\frac{1}{2 \alpha^{\prime}}} \rho(\theta)\left(\sigma^{\oplus n}\right)^{-\frac{1}{2 \alpha^{\prime}}}\right\|_{\alpha} \\
& =n^{\frac{1}{\alpha^{\prime}}} \inf _{\sigma \in \mathcal{S}(\mathcal{H})}\left(\sum_{\omega} P(\omega)\left\|\sigma^{\frac{1-\alpha}{2 \alpha}} \omega \sigma^{\frac{1-\alpha}{2 \alpha}}\right\|_{\alpha}^{\alpha}\right)^{\frac{1}{\alpha}},
\end{aligned}
$$

where $\frac{1}{\alpha}+\frac{1}{\alpha^{\prime}}=1$. Similarly, we have $\rho(0)=\oplus_{\omega} P(\omega)^{\frac{1}{\alpha_{0}}} \omega, \rho(1)=\oplus_{\omega} P(\omega)^{\frac{1}{\alpha_{1}}} \omega$ and

$$
\begin{aligned}
& \|\rho(0)\|_{L_{1}^{\alpha_{0}}(\mathcal{N} \subset \mathcal{M})}=n^{\frac{1}{\alpha_{0}^{\prime}}} \inf _{\sigma \in \mathcal{S}(\mathcal{H})}\left(\sum_{\omega} P(\omega) \| \sigma^{\left.\frac{1-\alpha_{0}}{2 \alpha_{0}} \omega \sigma^{\frac{1-\alpha_{0}}{2 \alpha_{0}}} \|_{\alpha_{0}}^{\alpha_{0}}\right)^{\frac{1}{\alpha_{0}}} ;}\right. \\
& \|\rho(1)\|_{L_{1}^{\alpha_{1}}(\mathcal{N} \subset \mathcal{M})}=n^{\frac{1}{\alpha_{1}^{\prime}}} \inf _{\sigma \in \mathcal{S}(\mathcal{H})}\left(\sum_{\omega} P(\omega)\left\|\sigma^{\frac{1-\alpha_{1}}{2 \alpha_{1}}} \omega \sigma^{\frac{1-\alpha_{1}}{2 \alpha_{1}}}\right\|_{\alpha_{1}}^{\alpha_{1}}\right)^{\frac{1}{\alpha_{1}}} .
\end{aligned}
$$

Because of the complex interpolation relation $L_{1}^{\alpha}(\mathcal{N} \subset \mathcal{M})=\left[L_{1}^{\alpha_{0}}(\mathcal{N} \subset \mathcal{M}), L_{1}^{\alpha_{1}}(\mathcal{N} \subset \mathcal{M})\right]_{\theta}$ mentioned in (14) of Section 3, we have the interpolation inequality (12):

$$
\|\rho(\theta)\|_{L_{1}^{\alpha}(\mathcal{N} \subset \mathcal{M})} \leq\left(\sup _{t \in \mathbb{R}}\|\rho(\mathrm{i} t)\|_{L_{1}^{\alpha_{0}(\mathcal{N} \subset \mathcal{M})}}\right)^{1-\theta}\left(\sup _{t \in \mathbb{R}}\|\rho(1+\mathrm{i} t)\|_{L_{1}^{\alpha_{1}}(\mathcal{N} \subset \mathcal{M})}\right)^{\theta}
$$

Now we claim that for all $\alpha \geq 1$ and $t \in \mathbb{R}$,

$$
\begin{aligned}
\|\rho(\mathrm{i} t)\|_{L_{1}^{\alpha}(\mathcal{N} \subset \mathcal{M})} & \leq\|\rho(0)\|_{L_{1}^{\alpha_{0}}(\mathcal{N} \subset \mathcal{M})} \\
\|\rho(1+\mathrm{i} t)\|_{L_{1}^{p}(\mathcal{N} \subset \mathcal{M})} & \leq\|\rho(1)\|_{L_{1}^{\alpha_{1}}(\mathcal{N} \subset \mathcal{M})}
\end{aligned}
$$

Write $X(\omega, t)=P(\omega)^{\frac{-\mathrm{i} t}{\alpha_{0}}+\frac{\mathrm{i} t}{\alpha_{1}}}$. Then, using Hölder inequality, it holds for all $p \geq 1$ and $t \in \mathbb{R}$ that,

$$
\begin{aligned}
\|\rho(\mathrm{i} t)\|_{L_{1}^{p}(\mathcal{N} \subset \mathcal{M})} & =\inf _{\sigma_{1}, \sigma_{2} \in \mathcal{S}(\mathcal{H})}\left\|\bigoplus_{\omega} X(t, \omega) P(\omega)^{\frac{1}{\alpha_{0}}} \sigma_{1}^{\frac{1-p}{2 p}} \omega \sigma_{2}^{\frac{1-p}{2 p}}\right\|_{p} \\
& \leq \inf _{\sigma_{1} \in \mathcal{S}(\mathcal{H})}\left\|\bigoplus_{\omega} X(t, \omega) P(\omega)^{\frac{1}{2 \alpha_{0}}} \sigma_{1}^{\frac{1-p}{2 p}} \omega^{\frac{1}{2}}\right\|_{2 p} \inf _{\sigma_{2} \in \mathcal{S}(\mathcal{H})}\left\|\bigoplus_{\omega} P(\omega)^{\frac{1}{2 \alpha_{0}}} \omega^{\frac{1}{2}} \sigma_{2}^{\frac{1-p}{2 p}}\right\|_{2 p} \\
& =\inf _{\sigma_{1} \in \mathcal{S}(\mathcal{H})}\left\|\bigoplus_{\omega} P(\omega)^{\frac{1}{\alpha_{0}}} \sigma_{1}^{\frac{1-p}{2 p}} \omega^{\frac{1}{2}} \sigma_{1}^{\frac{1-p}{2 p}}\right\|_{p}^{\frac{1}{2}} \inf _{\sigma_{2} \in \mathcal{S}(\mathcal{H})}\left\|\bigoplus_{\omega} P(\omega)^{\frac{1}{\alpha_{0}}} \sigma_{2}^{\frac{1-p}{2 p}} \omega^{\frac{1}{2}} \sigma_{2}^{\frac{1-p}{2 p}}\right\|_{p}^{\frac{1}{2}} \\
& =\|\rho(0)\|_{L_{1}^{p}(\mathcal{N} \subset \mathcal{M})}^{\frac{1}{2}}\|\rho(0)\|_{L_{1}^{p}(\mathcal{N} \subset \mathcal{M})}^{\frac{1}{2}}=\|\rho(0)\|_{L_{1}^{p}(\mathcal{N} \subset \mathcal{M})},
\end{aligned}
$$

where equality (30) holds since $X(t, \omega) \overline{X(t, \omega)}=1$. Then, the assertion in (29) follows similar argument. Therefore, combining (27), (28), and (29), we obtain that

$$
\|\rho(\theta)\|_{L_{1}^{\alpha}(\mathcal{N} \subset \mathcal{M})} \leq\|\rho(0)\|_{L_{1}^{\alpha_{0}}(\mathcal{N} \subset \mathcal{M})}^{\theta}\|\rho(1)\|_{L_{1}^{\alpha_{1}}(\mathcal{N} \subset \mathcal{M})}^{\theta} .
$$

which yields the desired (26). Hence, concavity $s \mapsto E_{0}^{r, *}(s, P)$ for $s \in(-1,0)$ is proved.

Next, we prove the concavity of $E_{0}^{*, a}(s, P)$ under the assumption $\mathcal{H}$ is finite-dimensional. By the continuity in the probability distribution $P$ from Proposition 5 -(c), it suffices to consider a finitely supported 
$P$ such that for each $\omega$, the probability $P(\omega)$ is rational. We can write $P(\omega)=\frac{n_{\omega}}{n}$ with some positive integers $n_{\omega}$ and $n=\sum_{\omega \in \mathcal{W}} n_{\omega}$. Given such a distribution $P$, we choose the following product state in $\mathcal{B}(\mathcal{H})^{\otimes n}$ :

$$
\begin{aligned}
\rho & :=\underbrace{W_{1} \otimes W_{1} \otimes \cdots \otimes W_{1}}_{n_{1} \text {-fold tensor }} \otimes \underbrace{W_{2} \otimes \cdots \otimes W_{2}}_{n_{2} \text {-fold tensor }} \otimes \cdots \otimes \underbrace{W_{k} \otimes \cdots \otimes W_{k}}_{n_{k} \text {-fold tensor }} \\
& =\left(W_{1}^{\otimes n_{1}}\right) \otimes\left(W_{2}^{\otimes n_{2}}\right) \otimes \cdots \otimes\left(W_{k}^{\otimes n_{k}}\right),
\end{aligned}
$$

where $\left\{W_{1}, W_{2}, \ldots, W_{k}\right\} \subseteq \mathcal{W}$. We have

$$
\begin{aligned}
I_{\alpha}^{\mathrm{a}, *}(P, \mathcal{W}) & =\inf _{\sigma \in \mathcal{S}(\mathcal{H})} \sum_{\omega \in \mathcal{W}} P(\omega) D_{\alpha}^{*}(\omega \| \sigma) \\
& =\inf _{\sigma \in \mathcal{S}(\mathcal{H})} \sum_{\omega \in \mathcal{W}} P(\omega) \alpha^{\prime} \log \left\|\sigma^{-\frac{1}{2 \alpha^{\prime}}} \omega \sigma^{-\frac{1}{2 \alpha^{\prime}}}\right\|_{\alpha} \\
& =\frac{\alpha^{\prime}}{n} \inf _{\sigma \in \mathcal{S}(\mathcal{H})} \log \left\|\sigma^{-\frac{1}{2 \alpha^{\prime}}} W_{1} \sigma^{-\frac{1}{2 \alpha^{\prime}}}\right\|_{\alpha}^{n_{1}} \cdot\left\|\sigma^{-\frac{1}{2 \alpha^{\prime}}} W_{2} \sigma^{-\frac{1}{2 \alpha^{\prime}}}\right\|_{\alpha}^{n_{2}} \cdots\left\|\sigma^{-\frac{1}{2 \alpha^{\prime}}} W_{k} \sigma^{-\frac{1}{2 \alpha^{\prime}}}\right\|_{\alpha}^{n_{k}} \\
& =\frac{\alpha^{\prime}}{n} \log \inf _{\sigma \in \mathcal{S}(\mathcal{H})}\left\|\left(\sigma^{\otimes n}\right)^{-\frac{1}{2 \alpha^{\prime}}} \rho\left(\sigma^{\otimes n}\right)^{-\frac{1}{2 \alpha^{\prime}}}\right\|_{\alpha}
\end{aligned}
$$

where $\rho$ is the density chosen in (31), and $\alpha^{\prime}$ is the conjugate of $\alpha$, i.e. $\frac{1}{\alpha}+\frac{1}{\alpha^{\prime}}=1$. Similar as for $E_{0}^{r, *}(s, P)$ it suffices to prove the following interpolation-type inequality: for all $1 \leq \alpha_{0}, \alpha_{1} \leq \infty, \frac{1}{\alpha}=\frac{1-\theta}{\alpha_{0}}+\frac{\theta}{\alpha_{1}}$, $\theta \in[0,1]$

$$
\begin{aligned}
& \inf _{\sigma \in \mathcal{S}(\mathcal{H})}\left\|\left(\sigma^{\otimes n}\right)^{-\frac{1}{2 \alpha^{\prime}}} \rho\left(\sigma^{\otimes n}\right)^{-\frac{1}{2 \alpha^{\prime}}}\right\|_{\alpha} \\
& \leq \inf _{\sigma \in \mathcal{S}(\mathcal{H})}\left\|\left(\sigma^{\otimes n}\right)^{-\frac{1}{2 \alpha_{0}^{\prime}}} \rho\left(\sigma^{\otimes n}\right)^{-\frac{1}{2 \alpha_{0}^{\prime}}}\right\|_{\alpha_{0}}^{1-\theta} \inf _{\sigma \in \mathcal{S}(\mathcal{H})}\left\|\left(\sigma^{\otimes n}\right)^{-\frac{1}{2 \alpha_{1}^{\prime}}} \rho\left(\sigma^{\otimes n}\right)^{-\frac{1}{2 \alpha_{1}^{\prime}}}\right\|_{\alpha_{1}}^{\theta} .
\end{aligned}
$$

Note that the product states $\sigma^{\otimes n}$ does not form a convex set; hence it is not the state space of a subalgebra. So for this case, the argument using amalgamated $L_{p}$ spaces does not apply. Instead, we provide a proof using direct interpolation. First,

$$
\inf _{\sigma \in \mathcal{S}(\mathcal{H})}\left\|\left(\sigma^{\otimes n}\right)^{-\frac{1}{2 \alpha^{\prime}}} \rho\left(\sigma^{\otimes n}\right)^{-\frac{1}{2 \alpha^{\prime}}}\right\|_{\alpha}=\inf _{\|\pi\|_{2 \alpha^{\prime}}=1}\left\|\left(\tau^{\otimes n}\right)^{-1} \rho\left(\tau^{\otimes n}\right)^{-1}\right\|_{\alpha} .
$$

where the infimum is taken over all positive $\tau$ with $\|\tau\|_{2 \alpha^{\prime}}=1$. Given $1 \leq \alpha_{0}, \alpha_{1} \leq \infty$ and $\epsilon>0$, we choose $\tau_{0}$ and $\tau_{1}$ such that $\left\|\tau_{0}\right\|_{2 \alpha_{0}^{\prime}}=\left\|\tau_{1}\right\|_{2 \alpha_{1}^{\prime}}=1$ and

$$
\begin{aligned}
& \left\|\left(\tau_{0}^{\otimes n}\right)^{-1} \rho\left(\tau_{0}^{\otimes n}\right)^{-1}\right\|_{\alpha_{0}} \leq(1+\epsilon) \inf _{\|\tau\|_{2 \alpha_{0}^{\prime}}=1}\left\|\left(\tau^{\otimes n}\right)^{-1} \rho\left(\tau^{\otimes n}\right)^{-1}\right\|_{\alpha_{0}}, \\
& \left\|\left(\tau_{1}^{\otimes n}\right)^{-1} \rho\left(\tau_{1}^{\otimes n}\right)^{-1}\right\|_{\alpha_{1}} \leq(1+\epsilon) \inf _{\|\tau\|_{2 \alpha_{1}^{\prime}=1}}\left\|\left(\tau^{\otimes n}\right)^{-1} \rho\left(\tau^{\otimes n}\right)^{-1}\right\|_{\alpha_{1}} .
\end{aligned}
$$

Because $\mathcal{H}$ is finite-dimensional, we can assume there is a $\delta>0$ such that $\tau_{0}, \tau_{1} \geq \delta \mathbb{1}$. Using Devinatz's factorization theorem [119] (see also Pisier's paper [120, Theorem 3.2]), there exists an operator valued analytic function $w:\{z \mid 0 \leq \operatorname{Re}(z) \leq 1\} \rightarrow \mathcal{B}(\mathcal{H})$ such that $w(z)$ is invertible for all $z$ with $z \mapsto w(z)^{-1}$ is bounded and analytic and moreover,

$$
w(\mathrm{i} t) w(\mathrm{i} t)^{*}=\tau_{0}^{2}, \quad w(1+\mathrm{i} t) w(1+\mathrm{i} t)^{*}=\tau_{1}^{2}, \quad \forall t \in \mathbb{R} .
$$

Then

$$
\begin{aligned}
& \|w(\mathrm{i} t)\|_{2 \alpha_{0}^{\prime}}=\left\|w(\mathrm{i} t) w(\mathrm{i} t)^{*}\right\|_{\alpha_{0}^{\prime}}^{\frac{1}{2}}=\left\|\tau_{0}^{2}\right\|_{\alpha_{0}^{\prime}}^{\frac{1}{2}}=\left\|\tau_{0}\right\|_{2 \alpha_{0}^{\prime}}=1, \\
& \|w(1+\mathrm{i} t)\|_{2 \alpha_{1}^{\prime}}=\left\|w(1+\mathrm{i} t) w(1+\mathrm{i} t)^{*}\right\|_{\alpha_{1}^{\prime}}^{\frac{1}{2}}=\left\|\tau_{1}^{2}\right\|_{\alpha_{1}^{\prime}}^{\frac{1}{2}}=\left\|\tau_{1}\right\|_{2 \alpha_{1}^{\prime}}=1
\end{aligned}
$$


which implies

$$
\|w(\theta)\|_{2 \alpha^{\prime}} \leq\left(\sup _{t}\|w(\mathrm{i} t)\|_{2 \alpha_{0}^{\prime}}\right)^{1-\theta}\left(\sup _{t}\|w(1+\mathrm{i} t)\|_{2 \alpha_{1}^{\prime}}\right)^{\theta}=1
$$

by interpolation inequality. Next, consider the analytic function

$$
f(z)=\rho^{\frac{1}{2}}(w(z) \otimes w(z) \otimes \cdots \otimes w(z))^{-1}, \quad z \in\{z \mid 0 \leq \operatorname{Re}(z) \leq 1\} .
$$

Note that for all $t \in \mathbb{R}$,

$$
\begin{aligned}
& \|f(\mathrm{i} t)\|_{2 \alpha_{0}}=\left\|\left(w^{*}(\mathrm{i} t)^{\otimes n}\right)^{-1} \rho\left(w(\mathrm{i} t)^{\otimes n}\right)^{-1}\right\|_{\alpha_{0}}=\left\|\left(\tau_{0}^{\otimes n}\right)^{-1} \rho\left(\tau_{0}^{\otimes n}\right)^{-1}\right\|_{\alpha_{0}}, \\
& \|f(1+\mathrm{i} t)\|_{2 \alpha_{1}}=\left\|\left(w^{*}(1+\mathrm{i} t)^{\otimes n}\right)^{-1} \rho\left(w(1+\mathrm{i} t)^{\otimes n}\right)^{-1}\right\|_{\alpha_{1}}=\left\|\left(\tau_{1}^{\otimes n}\right)^{-1} \rho\left(\tau_{1}^{\otimes n}\right)^{-1}\right\|_{\alpha_{1}},
\end{aligned}
$$

because the polar decomposition $w(\mathrm{i} t)=u(t) \tau_{0}^{\frac{1}{2}}, w(1+\mathrm{i} t)=v(t) \tau_{0}^{\frac{1}{2}}$ for some unitary function $u(t), v(t)$. Then by interpolation,

$$
\begin{aligned}
\|f(\theta)\|_{2 \alpha}^{2} & \leq\left(\sup _{t}\|f(\mathrm{i} t)\|_{2 \alpha_{0}}^{2}\right)^{1-\theta}\left(\sup _{t}\|f(1+\mathrm{i} t)\|_{2 \alpha_{1}}^{2}\right)^{\theta} \\
& =\left\|\left(\tau_{0}^{\otimes n}\right)^{-1} \rho\left(\tau_{0}^{\otimes n}\right)^{-1}\right\|_{\alpha_{0}}^{1-\theta}\left\|\left(\tau_{1}^{\otimes n}\right)^{-1} \rho\left(\tau_{1}^{\otimes n}\right)^{-1}\right\|_{\alpha_{1}}^{\theta} \\
& \leq(1+\epsilon)^{2}\left(\inf _{\|\tau\|_{2 \alpha_{0}^{\prime}=1}}\left\|\left(\tau^{\otimes n}\right)^{-1} \rho\left(\tau^{\otimes n}\right)^{-1}\right\|_{\alpha_{0}}\right)^{1-\theta}\left(\inf _{\|\tau\|_{2 \alpha_{1}^{\prime}=1}}\left\|\left(\tau^{\otimes n}\right)^{-1} \rho\left(\tau^{\otimes n}\right)^{-1}\right\|_{\alpha_{1}}\right)^{\theta} .
\end{aligned}
$$

Note that $\left\|w(\theta)^{*}\right\|_{2 \alpha^{\prime}} \leq 1$. Choosing $\tau=\frac{|w(\theta)|}{\|w(\theta)\|_{2 \alpha^{\prime}}}$,

$$
\begin{aligned}
\|f(\theta)\|_{2 \alpha}^{2} & =\left\|f(\theta) f(\theta)^{*}\right\|_{\alpha} \\
& =\left\|\left(w^{*}(\theta)^{\otimes n}\right)^{-1} \rho\left(w(\theta)^{\otimes n}\right)^{-1}\right\|_{\alpha} \\
& =\left\|\left(|w(\theta)|^{\otimes n}\right)^{-1} \rho\left(|w(\theta)|^{\otimes n}\right)^{-1}\right\|_{\alpha} \\
& =\left\|\left(\tau^{\otimes n}\right)^{-1} \rho\left(\tau^{\otimes n}\right)^{-1}\right\|_{\alpha}\|w(\theta)\|_{2 \alpha^{\prime}}^{-2} \\
& \geq\left\|\left(\tau^{\otimes n}\right)^{-1} \rho\left(\tau^{\otimes n}\right)^{-1}\right\|_{\alpha} \\
& \geq \inf _{\|t\|_{2 \alpha^{\prime}}=1}\left\|\left(\tau^{\otimes n}\right)^{-1} \rho\left(\tau^{\otimes n}\right)^{-1}\right\|_{\alpha} .
\end{aligned}
$$

Taking $\epsilon \rightarrow 0$, we obtain the desired interpolation inequality.

Next, we move on to prove the concavity of concavity of auxiliary functions of Petz form, i.e. $E_{0}^{\mathrm{r}}(s, P)$ and $E_{0}^{\mathrm{a}}(s, P)$. The main ingredients are the noncommutative Sibson's identity proved in Proposition 2 in Section 3 and Devinatz's factorization theorem [119].

Theorem 12 (Concavity of Petz Form in Order). Let $\mathcal{W} \subset \mathcal{S}(\mathcal{H})$ and $P \in \mathcal{P}(\mathcal{W})$ be a probability mass function.

(a) The map $s \mapsto E_{0}^{\mathrm{r}}(s, P)$ is concave on $(-1,0)$.

(b) If $\mathcal{H}$ is finite-dimensional, then $s \mapsto E_{0}^{\mathrm{a}}(s, P)$ is concave on $(-1,0)$.

Proof of Theorem 12. Note that

$$
\begin{aligned}
I_{\alpha}^{\mathrm{r}}(P, \mathcal{W}) & =\inf _{\sigma \in \mathcal{S}(\mathcal{H})} \frac{1}{\alpha-1} \log \left(\sum_{\omega} P(\omega) \operatorname{Tr}\left[\omega^{\alpha} \sigma^{1-\alpha}\right]\right) \\
& =\inf _{\sigma \in \mathcal{S}(\mathcal{H})} \frac{1}{\alpha-1} \log \left\|\bigoplus_{\omega} P^{\frac{1}{2}}(\omega) \omega^{\frac{\alpha}{2}} \sigma^{\frac{1-\alpha}{2}}\right\|_{2} .
\end{aligned}
$$


Hence, the concavity of $s \mapsto E_{0}(s, P)$ for $s \in(-1,0)$ is equivalent to showing that for all $\alpha_{0}, \alpha_{1} \geq 1$ with $\frac{1}{\alpha}=\frac{1-\theta}{\alpha_{0}}+\frac{\theta}{\alpha_{1}}, \theta \in[0,1]$, and $\sigma_{0}, \sigma_{1} \in \mathcal{S}(\mathcal{H})$,

$$
\inf _{\sigma \in \mathcal{S}(\mathcal{H})} \operatorname{Tr}\left[\left(\sum_{\omega} P(\omega) \omega^{\alpha}\right) \sigma^{1-\alpha}\right]^{\frac{1}{\alpha}} \leq\left\|\bigoplus_{\omega} P^{\frac{1}{2}}(\omega) \omega^{\frac{\alpha_{0}}{2}} \sigma_{0}^{\frac{1-\alpha_{0}}{2}}\right\|_{2}^{\frac{1-\theta}{\alpha_{0}}}\left\|\bigoplus_{\omega} P^{\frac{1}{2}}(\omega) \omega^{\frac{\alpha_{1}}{2}} \sigma_{1}^{\frac{1-\alpha_{1}}{2}}\right\|_{2}^{\frac{\theta}{\alpha_{1}}} .
$$

To prove this, we employ a similar argument as our proof of Proposition 3 in Section 3. Denote $\gamma=\frac{\alpha(1-\theta)}{\alpha_{0}}$ and $1-\gamma=\frac{\alpha \theta}{\alpha_{1}}$. Let us first consider the case $\gamma=\frac{1}{2}$ and $\alpha=\frac{1}{2}\left(\alpha_{0}+\alpha_{1}\right)$. Then, we start with CauchySchwartz inequality:

$$
\begin{aligned}
\left\|\bigoplus_{\omega} P^{\frac{1}{2}}(\omega) \omega^{\frac{\alpha_{0}}{2}} \sigma_{0}^{\frac{1-\alpha_{0}}{2}}\right\|_{2}\left\|\bigoplus_{\omega} P^{\frac{1}{2}}(\omega) \omega^{\frac{\alpha_{1}}{2}} \sigma_{1}^{\frac{1-\alpha_{1}}{2}}\right\|_{2} & \geq\left\|\bigoplus_{\omega} P(\omega) \sigma_{0}^{\frac{1-\alpha_{0}}{2}} \omega^{\frac{\alpha_{0}+\alpha_{1}}{2}} \sigma_{1}^{\frac{1-\alpha_{1}}{2}}\right\|_{1} \\
& \geq\left\|\mathbb{E}\left[\bigoplus_{\omega} P(\omega) \sigma_{0}^{\frac{1-\alpha_{0}}{2}} \omega^{\frac{\alpha_{0}+\alpha_{1}}{2}} \sigma_{1}^{\frac{1-\alpha_{1}}{2}}\right]\right\|_{1} \\
& =\left\|\left(\bigoplus_{\omega} \sigma_{0}^{\frac{1-\alpha_{0}}{2}}\right) \mathbb{E}\left[\bigoplus_{\omega} P(\omega) \omega^{\alpha}\right]\left(\bigoplus_{\omega} \sigma_{1}^{\frac{1-\alpha_{1}}{2}}\right)\right\|_{1} \\
& =\left\|\sigma_{0}^{\frac{1-\alpha_{0}}{2}}\left(\sum_{\omega} P(\omega) \omega^{\alpha}\right) \sigma_{1}^{\frac{1-\alpha_{1}}{2}}\right\|_{1} \\
& \geq\left\|\sum_{\omega} P(\omega) \omega^{\alpha}\right\| \|_{\frac{1}{\alpha}} \\
& =\inf _{\sigma \in \mathcal{S}(\mathcal{H})} \operatorname{Tr}\left[\left(\sum_{\omega} P(\omega) \omega^{\alpha}\right) \sigma^{1-\alpha}\right] .
\end{aligned}
$$

Here, we denote $\mathbb{E}$ as the conditional expectation from the algebra $\mathcal{M}:=\oplus_{x \in \mathcal{X}} \mathcal{B}(\mathcal{H})$ to the subalgebra $\mathcal{N}:=\mathbb{1}_{\mathcal{X}} \otimes \mathcal{B}(\mathcal{H})$ for $\mathcal{X}:=\operatorname{supp}(P)$, i.e.

$$
\mathbb{E}\left[\bigoplus_{x \in \mathcal{X}} \rho_{x}\right]=1 \mathcal{X} \otimes\left(\frac{1}{|\mathcal{X}|} \sum_{x \in \mathcal{X}} \rho_{x}\right), \quad \forall \rho_{x} \in \mathcal{B}(\mathcal{H})
$$

Hence, inequality (32) follows from the contraction of 1-norm under E. In Eq. (33), we employ the module property of $\mathbb{E}$, i.e.

$$
\mathbb{E}(a x b)=a \mathbb{E}(x) b, \quad \forall a, b \in \mathcal{N}, x \in \mathcal{M} .
$$

The last inequality (34) is owing to the Hölder inequality for $\alpha=1+\frac{\alpha_{0}-1}{2}+\frac{\alpha_{1}-1}{2}$ and the fact that $\sigma_{0}$ and $\sigma_{1}$ are density operators, i.e.

$$
\begin{aligned}
\left\|\sum_{\omega} P(\omega) \omega^{\alpha}\right\|_{\frac{1}{\alpha}} & \leq\left\|\sigma_{1}^{\frac{\alpha_{1}-1}{2}}\right\|_{\frac{2}{\alpha_{1}-1}}\left\|\sigma_{1}^{\frac{1-\alpha_{1}}{2}}\left(\sum_{\omega} P(\omega) \omega^{\alpha}\right) \sigma_{0}^{\frac{1-\alpha_{0}}{2}}\right\|_{1}\left\|\sigma_{0}^{\frac{\alpha_{0}-1}{2}}\right\|_{\frac{2}{\alpha_{0}-1}} \\
& \leq\left\|\sigma_{1}^{\frac{1-\alpha_{1}}{2}}\left(\sum_{\omega} P(\omega) \omega^{\alpha}\right) \sigma_{0}^{\frac{1-\alpha_{0}}{2}}\right\|_{1} .
\end{aligned}
$$

In the last equality (35), we apply the noncommutative Sibson's identity given in Proposition 2.

This proves the inequality for $\gamma=\frac{1}{2}$. Using induction, we obtain the inequality for $2^{n}$-partition points $\alpha=k 2^{-n}\left(\alpha_{1}-\alpha_{0}\right)+\alpha_{0}$ for all $k, n \in \mathbb{N}, 0 \leq k \leq 2^{n}$. The case for general $\alpha$ follows from the continuity.

Next, we prove the concavity of $s \mapsto E_{0}^{\mathrm{a}}(s, P)$. In the following, we assume that the Hilbert space $\mathcal{H}$ is finite-dimensional. Using the construction in Eqs. (31), it suffices to prove the following interpolation 
type inequality: for all $1 \leq \alpha_{0}, \alpha_{1} \leq \infty, \frac{1}{\alpha}=\frac{1-\theta}{\alpha_{0}}+\frac{\theta}{\alpha_{1}}, \theta \in[0,1]$,

$$
\inf _{\sigma \in \mathcal{S}(\mathcal{H})} \operatorname{Tr}\left[\rho^{\alpha}\left(\sigma^{\otimes n}\right)^{1-\alpha}\right]^{\frac{1}{\alpha}} \leq \inf _{\sigma \in \mathcal{S}(\mathcal{H})} \operatorname{Tr}\left[\rho^{\alpha_{0}}\left(\sigma^{\otimes n}\right)^{1-\alpha_{0}}\right]^{\frac{1-\theta}{\alpha_{0}}} . \inf _{\sigma \in \mathcal{S}(\mathcal{H})} \operatorname{Tr}\left[\rho^{\alpha_{1}}\left(\sigma^{\otimes n}\right)^{1-\alpha_{1}}\right]^{\frac{\theta}{\alpha_{1}}} .
$$

Note that

$$
\inf _{\sigma \in \mathcal{S}(\mathcal{H})} \operatorname{Tr}\left[\rho^{\alpha}\left(\sigma^{\otimes n}\right)^{1-\alpha}\right]=\inf _{\sigma \in \mathcal{S}(\mathcal{H})}\left\|\left(\sigma^{\otimes n}\right)^{\frac{1-\alpha}{2}} \rho^{\frac{\alpha}{2}}\right\|_{2}^{2}=\inf _{\|\tau\|_{\frac{2}{\alpha-1}}}\left\|\left(\tau^{\otimes n}\right)^{-1} \rho^{\frac{\alpha}{2}}\right\|_{2}^{2},
$$

where the infimum in the last line is taken over all invertible positive $\tau$ with $\|\tau\|_{\frac{2}{\alpha-1}}=1$. Letting $\lambda=\frac{\theta \alpha}{\alpha_{1}} \in[0,1]$, inequality $(36)$ can be rewritten as

$$
\inf _{\|\tau\|_{\frac{2}{\alpha-1}}} \|\left(\left\|\left(\tau^{\otimes n}\right)^{-1} \rho^{\frac{\alpha}{2}}\right\|_{2} \leq \inf _{\|\tau\|_{\frac{2}{\alpha_{0}-1}}}=1\left\|\left(\tau^{\otimes n}\right)^{-1} \rho^{\frac{\alpha_{0}}{2}}\right\|_{2}^{1-\lambda} \cdot \inf _{\|\tau\|_{\frac{2}{\alpha_{1}-1}}}\left\|\left(\tau^{\otimes n}\right)^{-1} \rho^{\frac{\alpha_{1}}{2}}\right\|_{2}^{\lambda} .\right.
$$

Given $1 \leq \alpha_{0}, \alpha_{1} \leq \infty$ and $\epsilon>0$, we choose $\tau_{0}$ and $\tau_{1}$ such that $\left\|\tau_{0}\right\|_{\frac{2}{\alpha_{0}-1}}=\left\|\tau_{1}\right\|_{\frac{2}{\alpha_{0}-1}}=1$ and

$$
\begin{aligned}
& \left\|\left(\tau_{0}^{\otimes n}\right)^{-1} \rho^{\frac{\alpha_{0}}{2}}\right\|_{2} \leq(1+\epsilon)_{\|\tau\|_{\frac{2}{\alpha_{0}-1}}} \inf _{\|}\left\|\left(\tau^{\otimes n}\right)^{-1} \rho^{\frac{\alpha_{0}}{2}}\right\|_{2}, \\
& \left\|\left(\tau_{1}^{\otimes n}\right)^{-1} \rho^{\frac{\alpha_{1}}{2}}\right\|_{2} \leq(1+\epsilon)_{\|\tau\|_{\frac{2}{\alpha_{1}-1}}} \inf \left\|\left(\tau^{\otimes n}\right)^{-1} \rho^{\frac{\alpha_{1}}{2}}\right\|_{2} .
\end{aligned}
$$

Using Devinatz's factorization theorem again as in the proof of Theorem 11, there exists an operator valued analytic function $w:\{z \mid 0 \leq \operatorname{Re}(z) \leq 1\} \rightarrow \mathcal{B}(\mathcal{H})$ such that $w(z)$ is invertible for all $z$ and

$$
w(\mathrm{i} t) w(\mathrm{i} t)^{*}=\tau_{0}^{2}, w(1+\mathrm{i} t) w(1+\mathrm{i} t)^{*}=\tau_{1}^{2}, \quad \forall t \in \mathbb{R} .
$$

Then

$$
\begin{aligned}
& \|w(\mathrm{i} t)\|_{\frac{2}{\alpha_{0}-1}}=\left\|w(\mathrm{i} t) w(\mathrm{i} t)^{*}\right\|_{\frac{1}{\alpha_{0}-1}}^{\frac{1}{2}}=\left\|\tau_{0}^{2}\right\|_{\frac{1}{\alpha_{0}-1}}^{\frac{1}{2}}=\left\|\tau_{0}\right\|_{\frac{2}{\alpha_{0}-1}}=1 \\
& \|w(1+\mathrm{i} t)\|_{\frac{2}{\alpha_{1}-1}}=\left\|w(1+\mathrm{i} t) w(1+\mathrm{i} t)^{*}\right\|_{\frac{1}{\alpha_{1}-1}}^{\frac{1}{2}}=\left\|\tau_{1}^{2}\right\|_{\frac{1}{\alpha_{1}-1}}^{\frac{1}{2}}=\left\|\tau_{1}\right\|_{\frac{2}{\alpha_{1}-1}}=1 .
\end{aligned}
$$

Using $\frac{1}{\alpha}=\frac{1-\theta}{\alpha_{0}}+\frac{\theta}{\alpha_{1}}$ and $\lambda=\frac{\theta \alpha}{\alpha_{1}}$, one can verify that

$$
\frac{\alpha_{0}-1}{2}(1-\lambda)+\frac{\alpha_{1}-1}{2} \lambda=\frac{\alpha-1}{2} .
$$

Note that for $1<\alpha<\infty, 0<\frac{2}{\alpha-1} \leq \infty$. The interpolation inequality also holds for $L_{p}, 0<p<\infty[121$, Lemma 2.5]. We have

$$
\|w(\lambda)\|_{\frac{2}{\alpha-1}} \leq\left(\sup _{t}\|w(\mathrm{i} t)\|_{\frac{2}{\alpha_{0}-1}}\right)^{1-\lambda}\left(\sup _{t}\|w(1+\mathrm{i} t)\|_{\frac{2}{\alpha_{1}-1}}\right)^{\lambda}=1 .
$$

Next, consider the analytic function

$$
f(z)=\rho^{\frac{\alpha_{0}}{2}+\frac{\alpha_{1}-\alpha_{0}}{2} z}(w(z) \otimes w(z) \otimes \cdots \otimes w(z))^{-1}, \quad z \in\{z \mid 0 \leq \operatorname{Re}(z) \leq 1\} .
$$

Note that for all $t \in \mathbb{R}$,

$$
\begin{aligned}
& \left.\|f(\mathrm{i} t)\|_{2}=\| \rho^{\frac{\alpha_{0}}{2}+\frac{\alpha_{1}-\alpha_{0}}{2}(\mathrm{i} t)} w(\mathrm{i} t)^{\otimes n}\right)^{-1}\left\|_{2}=\right\|\left(\tau_{0}^{\otimes n}\right)^{-1} \rho^{\frac{\alpha_{0}}{2}} \|_{2}, \\
& \|f(1+\mathrm{i} t)\|_{2}=\left\|\rho^{\frac{\alpha_{0}}{2}+\frac{\alpha_{1}-\alpha_{0}}{2}(1+\mathrm{i} t)}\right\|_{2}=\left\|\left(\tau_{1}^{\otimes n}\right)^{-1} \rho^{\frac{\alpha_{1}}{2}}\left(w(1+\mathrm{i} t)^{\otimes n}\right)^{-1}\right\|_{2},
\end{aligned}
$$


because the polar decomposition $w(\mathrm{i} t)=u(t) \sigma_{0}^{\frac{1}{2}}, w(1+\mathrm{i} t)=v(t) \sigma_{0}^{\frac{1}{2}}$ for some unitary function $u(t), v(t)$. By interpolation inequality again,

$$
\begin{aligned}
\|f(\lambda)\|_{2} & \leq\left(\sup _{t \in \mathbb{R}}\|f(\mathrm{i} t)\|_{2}\right)^{1-\lambda}\left(\sup _{t \in \mathbb{R}}\|f(1+\mathrm{i} t)\|_{2}\right)^{\lambda} \\
& =\left\|\left(\tau_{0}^{\otimes n}\right)^{-1} \rho^{\frac{\alpha_{0}}{2}}\right\|_{2}^{1-\lambda}\left\|\left(\tau_{1}^{\otimes n}\right)^{-1} \rho^{\frac{\alpha_{1}}{2}}\right\|_{2}^{\lambda} \\
& \leq(1+\epsilon)^{2}\left(\inf _{\|\tau\|_{\frac{2}{\alpha_{0}-1}}=1}\left\|\left(\tau^{\otimes n}\right)^{-1} \rho^{\frac{\alpha_{0}}{2}}\right\|_{2}\right)^{1-\lambda}\left(\inf _{\|\tau\|_{\frac{2}{\alpha_{1}-1}}=1}\left\|\left(\tau^{\otimes n}\right)^{-1} \rho^{\frac{\alpha_{1}}{2}}\right\|_{2}\right)^{\lambda} .
\end{aligned}
$$

On the other hand, by $\|w(\lambda)\|_{\frac{2}{\alpha-1}} \leq 1$ and choosing $\tau=\frac{|w(\lambda)|}{\| w(\lambda) \mid \frac{2}{\alpha-1}}$,

$$
\begin{aligned}
\|f(\lambda)\|_{2} & =\left\|\rho^{\frac{\alpha_{0}}{2}+\frac{\alpha_{1}-\alpha_{0}}{2} \lambda}\left(w(\lambda)^{\otimes n}\right)^{-1}\right\|_{2} \\
& =\left\|\rho^{\frac{\alpha}{2}}\left(w(\lambda)^{\otimes n}\right)^{-1}\right\|_{2} \\
& =\left\|\rho^{\frac{\alpha}{2}}\left(|w(\lambda)|^{\otimes n}\right)^{-1}\right\|_{2} \\
& =\left\|\rho^{\frac{\alpha}{2}}\left(\tau^{\otimes n}\right)^{-1}\right\|_{2}\|w(\lambda)\|_{\frac{2}{\alpha-1}}^{-1} \\
& \geq\left\|\rho^{\frac{\alpha}{2}}\left(\tau^{\otimes n}\right)^{-1}\right\|_{2} \\
& \geq \inf \underset{\frac{2}{\alpha-1}}{\inf }\left\|\rho^{\frac{\alpha}{2}}\left(\tau^{\otimes n}\right)^{-1}\right\|_{2} .
\end{aligned}
$$

Taking $\epsilon \rightarrow 0$, we obtain the desired interpolation inequality.

Remark 5.2. The above concavity remains true if $\mathcal{B}(\mathcal{H})$ is replaced by tracial von Neumann algebras. In particular, the argument $E_{0}^{r, *}$ and $E_{0}^{r}$ extends for all semifinite von Neumann algebra, and the argument $E_{0}^{\mathrm{a}, *}$ and $E_{0}^{\mathrm{a}}$ works for all finite von Neumann algebra. Here the obstruction to extends the concavity of $E_{0}^{a}$ to the infinite-dimensional (semifinie) case is that Devinatz's factorization theorem [119] in our setting requires densities $\tau_{0}, \tau_{1} \geq \delta \mathbb{1}$ for some $\delta>0$. However, in the infinite dimensions, a density is bounded from below if and only if it is finite rank (its supported has finite trace). It is not known to us that the auxiliary functions can be approximated by finite rank densities.

Finally, we apply the established concavity properties in Theorem 11 and 12 and the equicontinuity of Rényi and Augustin information in prior input distributions (Propositions 4 and 5) to prove the following joint continuity.

Theorem 13 (Joint Continuity for Auxiliary Functions). Let $\mathcal{W} \subset \mathcal{S}(\mathcal{H})$. Assume $C_{\frac{1}{1+z}, \mathcal{W}}<\infty$ for some $z>-1$. The following holds.

(a) (Petz) The map $(s, P) \mapsto E_{0}^{r}(s, P)$ is jointly continuous on $[\max \{0, z\}, \infty] \times \mathcal{P}(\mathcal{W})$, and $(s, P) \mapsto$ $E_{0}^{\mathrm{a}}(s, P)$ is jointly continuous on $[z, 0) \times \mathcal{P}(\mathcal{W})$ and $(0, \infty] \times \mathcal{P}(\mathcal{W})$.

(b) (Sandwiched) The maps $(s, P) \mapsto E_{0}^{\mathrm{r}, *}(s, P)$ and $(s, P) \mapsto E_{0}^{\mathrm{a}, *}(s, P)$ are jointly continuous on $[z, 0) \times \mathcal{P}(\mathcal{W})$ and $(0,1] \times \mathcal{P}(\mathcal{W})$, respectively

(c) (Log-Euclidean) The maps $(s, P) \mapsto E_{0}^{\mathrm{r}, \boldsymbol{b}}(s, P)$ and $(s, P) \mapsto E_{0}^{\mathrm{a}, b}(s, P)$ are jointly continuous on $[z, 0) \times \mathcal{P}(\mathcal{W})$ and $(0, \infty] \times \mathcal{P}(\mathcal{W})$, respectively

The assertions for $E_{0}^{\mathrm{a}}$ and $E_{0}^{\mathrm{a}, *}$ require an additional assumption of $|\mathcal{H}|<\infty$.

Remark 5.3. Note that $z=-1$ if $|\mathcal{H}|<\infty$. The technical assumption of $|\mathcal{H}|<\infty$ is because of Theorems 11-(b) and 12-(b). We conjecture that such the condition could be removed.

Remark 5.4. The concavity at $s=0$ is excluded in Theorem 13 (except for $E_{0}^{r}$ ) since we do not know if the associated auxiliary functions are differentiable at $s=0$. We leave this as an open problem in Section 7 . 
Nevertheless, the regions of interest are either $s \in(-1,0)$ or $s \in(0, \infty)$ as they correspond to the strong converse regime and the error exponent region of classical-quantum channel coding and classical data compression with quantum side information as we will discuss in Section 6 below.

Proof of Theorem 13. The concavity of $s \mapsto E_{0}^{\mathrm{r},(t)}(s, P)$ and $s \mapsto E_{0}^{\mathrm{a},(t)}(s, P)$ in $[0, \infty]$ obtained in Corollary B.2, and Proposition B.5 of [86] already imply that they are also continuous in $s \in[0, \infty]$. On the other hand, the established Theorems 11 and 12 imply that they are continuous in $s \in(-1,0)$. Recalling the definitions given in Eq. (24) and (25), one of the assertions follows from the continuity in $s$ and the equicontinuity in $P$ proven in Proposition 4-(c) and Proposition 5-(c).

\section{Applications of Auxiliary Functions in Error Exponent Analysis}

Before commencing the section, we first introduce the two relevant information-processing tasks- the classical-quantum (c-q) channel coding and the classical source coding with quantum side information (QSI). Then, we explain the roles of the auxiliary functions in error exponent analysis in these tasks.

6.1. Quantum Information-Processing Tasks. In the problems of c-q channel coding, the aim is to transmit classical information through a c-q channel with some coding strategy. The classical information is represented by the messages in a finite message set, which we denote by $\mathcal{I}$. An ( $n$-block) encoder is a map from the message set to an $n$-fold input alphabet $\mathcal{X}$, i.e. $f_{n}: \mathcal{I} \rightarrow \mathcal{X}^{n}$, such that each message $m \in \mathcal{I}$ is encoded to a codeword $\mathbf{x}^{n}(m):=x_{1}(m) x_{2}(m) \ldots x_{n}(m) \in \mathcal{X}^{n}$. A classical-quantum (c-q) channel $\mathcal{W}: x \mapsto W_{x}$ is a map from symbols in the input alphabet $\mathcal{X}$ to a density operator on the output alphabet, which is conventionally modeled by some Hilbert space $B \equiv \mathcal{H}_{B}$. Moreover, $n$-blocklength codeword $\mathbf{x}^{n}(m)$ through the c-q channel will be mapped to a product state:

$$
\mathcal{W}: \mathbf{x}^{n}(m) \mapsto W_{\mathbf{x}^{n}(m)}^{\otimes n}=W_{x_{1}(m)} \otimes W_{x_{2}(m)} \otimes \cdots \otimes W_{x_{n}(m)} \in \mathcal{S}(B)^{\otimes n} .
$$

The decoder $\mathcal{D}_{n}$ is described by a positive operator-valued measurement (POVM) $\Pi=\left\{\Pi_{1}, \ldots, \Pi_{|\mathcal{I}|}\right\}$ on $\mathcal{H}^{\otimes n}$, where $\Pi_{i} \geq 0$ and $\sum_{i=1}^{|\mathcal{I}|} \Pi_{i}=\mathbb{1}$. The pair $\left(\mathcal{E}_{n}, \mathcal{D}_{n}\right)=: \mathcal{C}_{n}$ is called an $(n, R)$-code with transmission rate $R=\frac{1}{n} \log \left|\mathcal{C}_{n}\right|=\frac{1}{n} \log |\mathcal{I}|$. The error probability of sending a message $m$ with the code $\mathcal{C}_{n}$ is $\varepsilon_{m}\left(\mathcal{C}_{n}\right):=1-\operatorname{Tr}\left(\Pi_{m} W_{\mathbf{x}^{n}(m)}^{\otimes n}\right)$. The average error probability is defined by $\bar{\varepsilon}_{\mathrm{c}}\left(\mathcal{C}_{n}\right)=\frac{1}{|\mathcal{I}|} \sum_{m \in \mathcal{I}} \varepsilon_{m}\left(\mathcal{C}_{n}\right)$. We denote by $\varepsilon_{\mathrm{c}}^{\star}(n, R)$ the minimum average probability of error among all the channel coding strategies with a blocklength $n$ and transmission rate $R$, i.e.

$$
\varepsilon_{\mathrm{c}}^{\star}(n, R):=\inf \left\{\bar{\varepsilon}_{\mathrm{c}}\left(\mathcal{C}_{n}\right): \mathcal{C}_{n} \text { is an }(n, R) \text {-code }\right\},
$$

where the subscript ' $c$ ' is used to indicate that the underlying protocol is channel coding. A constant composition code with a composition (or the so-called type) $Q$ refers to a codebook whose codewords all have the same empirical distribution $Q$, i.e.

$$
\frac{1}{n} \sum_{i=1}^{n} \mathbf{1}_{\left\{x=x_{i}\right\}}=Q(x), \quad \forall x \in \mathcal{X},
$$

for any indicator function $\mathbf{1}$. We denote by $\varepsilon_{\mathrm{c}}^{\star}(n, R, Q)$ the minimum average probability of error over all $n$-blocklength constant composition codes with type $Q$ and transmission rate $R$, i.e.

$$
\varepsilon_{\mathrm{c}}^{\star}(n, R, Q):=\inf \left\{\bar{\varepsilon}_{\mathrm{c}}\left(\mathcal{C}_{n}\right): \mathcal{C}_{n} \text { is an }(n, R) \text {-code with type } Q\right\} .
$$

In the problems of classical source coding with QSI (or called the classical-quantum Slepian-Wolf coding), the aim is to compress classical data and decompress them with the aid of QSI. The classical data are represented by $n$-length sequence $\mathbf{x}^{n} \in \mathcal{X}^{\otimes n}$. The sequence can be produced from an identical and independently distributed (i.i.d.) probability distribution $P \in \mathcal{P}(\mathcal{X})$, i.e.

$$
\operatorname{Pr}\left(\mathbf{x}^{n}\right)=\left\{\begin{array}{ll}
\prod_{i=1}^{n} P\left(x_{i}\right) & \text { sources with i.i.d. } P \\
\frac{1}{T_{Q}^{n} \mid} \mathbf{1}_{\left\{\mathbf{x}^{n} \in T_{Q}^{n}\right\}} & \text { sources with type } Q
\end{array},\right.
$$

where we denote by $T_{Q}^{n}$ the type class that contains all $n$-length sequences with type $Q$. 
The encoder $\mathcal{E}_{n}: \mathcal{X}^{n} \rightarrow \mathcal{I}$ maps the source to a finite index set $\mathcal{I}$ with compression rate $R:=\frac{1}{n} \log |\mathcal{I}|$. The QSI

$$
\rho_{B^{n}}^{\mathbf{x}^{n}}:=\rho_{B}^{x_{1}} \otimes \rho_{B}^{x_{2}} \otimes \cdots \otimes \rho_{B}^{x_{n}} \in \mathcal{S}(B)^{\otimes n}
$$

is a product state and can be viewed as a c-q channel applied on the sequence as described in Eq. (37). For the case of i.i.d. source, the joint distribution that governs the sources and QSI can be modeled as a so-called c-q state $\rho_{X B}:=\sum_{x \in \mathcal{X}} P(x)|x\rangle\langle x| \otimes \rho_{B}^{x} \in \mathcal{S}(X B)$. This is nothing but the joint measure $P \circ \mathcal{W}$ described above when $\mathcal{W}: x \mapsto \rho_{B}^{x} \in \mathcal{S}(B)$. The decoder $\mathcal{D}_{n}$ is a family of POVM $\left\{\Pi_{\mathrm{x}^{n}}^{(i)}\right\}_{i \in \mathcal{I}}$ that receive the index $i \in \mathcal{I}$ and the corresponding density operator $\rho_{B^{n}}^{\mathbf{x}^{n}}$ to reproduce the source $\hat{\mathbf{x}}^{n}$. The error probability of the code $\mathcal{C}_{n}=\left(\mathcal{E}_{n}, \mathcal{D}_{n}\right)$ is thus

$$
\varepsilon_{\mathbf{S}}\left(\mathcal{C}_{n}\right):=\operatorname{Pr}\left(\hat{\mathbf{x}}^{n} \neq \mathbf{x}^{n}\right)=\sum_{\mathbf{x}^{n} \in \mathcal{X}^{n}} \operatorname{Pr}\left(\mathbf{x}^{n}\right) \operatorname{Tr}\left[\rho_{B^{n}}^{\mathbf{x}^{n}} \Pi_{\mathbf{x}^{n}}^{\left(\mathcal{E}_{n}\left(\mathbf{x}^{n}\right)\right)}\right] .
$$

We denote by $\varepsilon_{\mathrm{s}}^{\star}(n, R)$ the minimum average probability of error among all $n$-length source codes and compression rate $R$, i.e.

$$
\varepsilon_{\mathrm{s}}^{\star}(n, R):=\inf \left\{\bar{\varepsilon}_{\mathrm{s}}\left(\mathcal{C}_{n}\right): \mathcal{C}_{n} \text { is an }(n, R) \text {-code with i.i.d. source } P\right\},
$$

where the subscript ' $\mathrm{s}$ ' is used to indicate that the underlying protocol is source coding. Similarly, we denote by $\varepsilon_{\mathrm{s}}^{\star}(n, R, Q)$ the minimum average probability of error over all $n$-length source codes with type $Q$ and transmission rate $R$, i.e.

$$
\varepsilon_{\mathrm{s}}^{\star}(n, R, Q):=\inf \left\{\bar{\varepsilon}_{\mathrm{s}}\left(\mathcal{C}_{n}\right): \mathcal{C}_{n} \text { is an }(n, R, Q) \text {-code with type } Q\right\} .
$$

6.2. Error Exponent Analysis with Auxiliary Functions. One of the fundamental and critical problems in quantum information theory is to characterize the exponent of $\varepsilon_{\mathrm{c}}$ in terms of the coding blocklength and a fixed rate (We refer the reader to an exposition in Ref. [79]). Burnashev and Holevo $[88,89]$ first proved a random coding bound for pure-state channels (i.e. the channel output consists of rank-one density operators):

$$
-\frac{1}{n} \log \varepsilon_{\mathrm{c}}^{\star}(n, R) \geq \sup _{P \in \mathcal{P}(\mathcal{X})} \sup _{0 \leq s \leq 1}\left\{E_{0}^{\mathrm{r}}(s, P)-s R\right\}-\frac{1}{n} \log 4, \quad \forall n \in \mathbb{N} .
$$

Here, the auxiliary function $E_{0}^{r}$ is defined in Eq. (24) with Petz's Rényi divergence [80]. We note that the c-q channel $\mathcal{W}: x \mapsto W_{x}$ can be viewed as a collection of density operators indexed by $x \in \mathcal{X}$. Hence, the definitions given in Eqs. (24) and (25) naturally apply. The achievability bound in Eq. (38) was conjectured to hold for general classical-quantum channels. However, it is still open.

For the optimality (i.e. lower bound of $\varepsilon_{\mathrm{c}}$ ), Winter [92] employed a dummy channel method by Haroutunian [43] to prove a sphere-packing bound for c-q channel:

$$
\lim _{n \rightarrow \infty}-\frac{1}{n} \log \varepsilon_{\mathrm{c}}^{\star}(n, R) \leq \sup _{P \in \mathcal{P}(\mathcal{X})} \inf _{\mathcal{X}} \operatorname{\mathcal {X}}_{\rightarrow \mathcal{S}(B)}\left\{\sum_{x} P(x) D\left(V_{x} \| W_{x}\right): I(P, \mathcal{V}) \leq R\right\} .
$$

We note that the quantity before optimizing for all $P$ equals Csiszár's expression [47] in Eq. (7). Via a variational representation, the right-hand side of Winter's bound can be rewritten in terms of the auxiliary function $E_{0}^{\mathrm{a}, \mathrm{b}}$ defined by the log-Euclidean Rényi divergence:

$$
\sup _{P \in \mathcal{P}(\mathcal{X})} \sup _{s \geq 1}\left\{E_{0}^{\mathrm{a}, b}(s, P)-s R\right\} \text {. }
$$

Later, Dalai [93] generalized the approach by Shannon, Gallager, and Berlekamp [20, 21] to prove another version of sphere-packing bound for c-q channels:

$$
\lim _{n \rightarrow \infty}-\frac{1}{n} \log \varepsilon_{\mathrm{c}}^{\star}(R) \leq \sup _{P \in \mathcal{P}(\mathcal{X})} \sup _{s \geq 0}\left\{E_{0}^{\mathrm{r}}(s, P)-s R\right\} .
$$

Part of the authors further refined Dalai's result to the finite blocklength regime with higher-order terms of order $O\left(\frac{\log n}{n}\right)$. 
According to Lemma 1-(f) and Eq. (22), we have, for all $s \geq 0$,

$$
\sup _{P \in \mathcal{P}(\mathcal{X})} E_{0}^{\mathrm{r}}(s, P) \leq \sup _{P \in \mathcal{P}(\mathcal{X})} E_{0}^{\mathrm{a}, b}(s, P)
$$

Hence, the entropic exponent defined with Petz's version is tighter in the optimality bound. Moreover, the right-hand side of Eqs. (39) and (38) coincides when $R \geq R_{\text {crit }}$, where the critical rate $R_{\text {crit }}$ is the rate at which the slope of the right-hand side of (39) is -1 . That is the reason why the entropic exponent with Petz's version is believed to be the optimal error exponent. Let us emphasize again that the achievability bound given in (38) holds only for pure-state channels

If we restrict the channel codes to have a fixed type $P$, it is proved that the entropic exponent function defined in terms of $E_{0}^{\mathrm{a}}$ gives an upper bound to the exponent of $\varepsilon_{\mathrm{c}}^{\star}(n, R, P)[94,52]$ :

$$
-\frac{1}{n} \log \varepsilon_{\mathrm{c}}^{\star}(n, R, P) \leq \sup _{s \geq 1}\left\{E_{0}^{\mathrm{a}}(s, P)-s R\right\}+O\left(\frac{\log }{n}\right),
$$

where the higher-order term can be explicitly determined in [52]. However, it is still open for general codes even in the classical case.

In the strong converse region $\left(R>C_{\mathcal{W}}\right)$, the operational strong converse exponent has been determined by Mosonyi and Ogawa [86]:

$$
\begin{aligned}
\lim _{n \rightarrow \infty}-\frac{1}{n} \log \left[1-\varepsilon_{\mathrm{c}}^{\star}(n, R)\right] & =\inf _{P \in \mathcal{P}(\mathcal{X})} \sup _{-1<s<0}\left\{E_{0}^{\mathrm{r}, *}(s, P)-s R\right\} \\
& =\inf _{P \in \mathcal{P}(\mathcal{X})} \sup _{-1<s<0}\left\{E_{0}^{\mathrm{a}, *}(s, P)-s R\right\} .
\end{aligned}
$$

Note that the auxiliary functions in the above equality have been switched to the sandwiched version.

Recently, the finite blocklength sphere-packing bound was proved for the classical source coding with QSI as well $[54,55]$. For rate greater the compression limit, i.e. $R>H(X \mid B)_{\rho}:=-D\left(\rho_{X B} \| \mathbb{1}_{X} \otimes \rho_{B}\right)$, the bound for sources with a fixed type $Q$ is,

$$
-\frac{1}{n} \log \varepsilon_{\mathrm{s}}^{\star}(n, R, P) \leq \sup _{s \geq 0}\left\{E_{0, \mathrm{~s}}(s, P)+s R\right\}+O\left(\frac{\log }{n}\right)
$$

and for i.i.d. sources is,

$$
-\frac{1}{n} \log \varepsilon_{\mathrm{s}}^{\star}(n, R) \leq \sup _{s \geq 0}\left\{E_{0, \mathrm{~s}}(s)+s R\right\}+O\left(\frac{\log }{n}\right) .
$$

Here, the auxiliary functions for source coding with QSI are defined as follows [54, 55]:

$$
\begin{aligned}
E_{0, \mathrm{~s}}^{(t)}(s) & :=-s H_{\frac{1}{1+s}}^{(t)}(X \mid B)_{\rho} \\
E_{0, \mathrm{~s}}^{(t)}(s, P) & :=E_{0, \mathrm{c}}^{\mathrm{a},(t)}(s, P)-s H(P) \\
H_{\alpha}^{(t)}(X \mid B)_{\rho} & :=-\inf _{\sigma \in \mathcal{S}(B)} D_{\alpha}\left(\rho_{X B} \| \mathbb{1}_{X} \otimes \sigma\right) .
\end{aligned}
$$

The operational strong converse exponent (when $R<H(X \mid B)_{\rho}$ ) has been completely determined in terms of the sandwiched version [54]:

$$
\lim _{n \rightarrow \infty}-\frac{1}{n} \log \left[1-\varepsilon_{\mathrm{s}}^{\star}(n, R)\right]=\sup _{-1<s<0}\left\{E_{0, \mathrm{~s}}^{*}(s)+s R\right\} .
$$


To ease the burden of notation, we define the following entropic functions for classical-quantum channel coding and classical source coding with QSI, respectively: for $R \geq 0$ and $P \in \mathcal{P}(\mathcal{X})$,

$$
\begin{aligned}
E_{\mathrm{c}}(R, P) & :=\sup _{s>-1}\left\{E_{0}^{\mathrm{a}}(R, P) \vee E_{0}^{\mathrm{a}, *}(R, P)-s R\right\}, \\
E_{\mathrm{c}}(R) & := \begin{cases}\sup _{P \in \mathcal{P}(\mathcal{X})} E_{\mathrm{c}}(R, P), \quad R \leq C_{\mathcal{W}} \\
\inf _{P \in \mathcal{P}(\mathcal{X})} E_{\mathrm{c}}(R, P), \quad R>C_{\mathcal{W}},\end{cases} \\
E_{\mathrm{s}}(R, P) & :=\sup _{s>-1}\left\{E_{0, \mathrm{~s}}(R, P) \vee E_{0, \mathrm{~s}}^{*}(R, P)+s R\right\}, \\
E_{\mathrm{s}}(R) & :=\sup _{s>-1}\left\{E_{0, \mathrm{~s}}(R) \vee E_{0, \mathrm{~s}}^{*}(R)+s R\right\} .
\end{aligned}
$$

In the following, we collect applications of the properties of the auxiliary functions established in Section 5. Firstly, in Proposition 14 we prove a minimax identity for the strong converse exponent in classical-quantum channel coding.

Proposition 14 (A Minimax Identity in the Strong Converse Regime). For every $R>C_{\mathcal{W}}$,

$$
\begin{aligned}
E_{\mathrm{c}}(R) & =\inf _{P \in \mathcal{P}(\mathcal{X})} \sup _{-1<s<0}\left\{E_{0}^{\mathrm{r}, *}(s, P)-s R\right\}=\sup _{-1<s<0} \inf _{P \in \mathcal{P}(\mathcal{X})}\left\{E_{0}^{\mathrm{r}, *}(s, P)-s R\right\} \\
& =\inf _{P \in \mathcal{P}(\mathcal{X})} \sup _{-1<s<0}\left\{E_{0}^{\mathrm{a}, *}(s, P)-s R\right\}=\sup _{-1<s<0} \inf _{P \in \mathcal{P}(\mathcal{X})}\left\{E_{0}^{\mathrm{a}, *}(s, P)-s R\right\} .
\end{aligned}
$$

Note that in a recent paper [122], Mosonyi and Ogawa proved an expression for the strong converse exponent of constant composition with type $P$ :

$$
\lim _{n \rightarrow \infty}-\frac{1}{n} \log \left[1-\varepsilon_{\mathrm{c}}^{\star}(n, R, P)\right]=E_{\mathrm{c}}(R, P), \quad R>C_{\mathcal{W}} .
$$

The above result together with the established Proposition 14 then imply an important consequence. Namely, the strong converse exponent (over all possible codes) can be asymptotically attained by the best constant composition code, i.e. for $R>C_{\mathcal{W}}$,

$$
\lim _{n \rightarrow \infty}-\frac{1}{n} \log \left[1-\varepsilon_{\mathrm{c}}^{\star}(n, R)\right]=E_{\mathrm{c}}(R)=\inf _{P \in \mathcal{P}(\mathcal{X})} E_{\mathrm{c}}(R, P)=\lim _{n \rightarrow \infty} \inf _{\text {type } Q}-\frac{1}{n} \log \left[1-\varepsilon_{\mathrm{c}}^{\star}(n, R, Q)\right] .
$$

This gives classical-quantum channel coding with constant composition codes an operational meaning in the strong converse regime.

Secondly, the joint continuity properties provided in Theorem 13 and Berge's maximum theorem [123, Section IV.3], [124, Lemma 3.1] show that the entropic exponent functions introduced before are jointly continuous. Such the joint continuity property is crucial in the variable-length source coding with quantum side information [55] and finite blocklength analysis.

Proposition 15 (Joint Continuity for Entropic Exponents). Suppose $|\mathcal{H}|<\infty$. The following hold.

(a) The map $(R, P) \mapsto E_{\mathrm{c}}(R, P)$ is jointly continuous on $\left(I_{0}^{\mathrm{a}}(P, \mathcal{W}), I_{\infty}^{\mathrm{a}, *}(P, \mathcal{W})\right] \times \mathcal{P}(\mathcal{X})$, and $R \mapsto$ $E_{\mathrm{c}}(R)$ is continuous on $\left(I_{0}^{\mathrm{a}}(P, \mathcal{W}), I_{\infty}^{\mathrm{a}, *}(P, \mathcal{W})\right]$.

(b) The map $(R, P) \mapsto E_{\mathrm{s}}(R, P)$ is jointly continuous on $\left[H(P)-, I_{\infty}^{\mathrm{a}, *}(P, \mathcal{W}), H(P)-, I_{0}^{\mathrm{a}}(P, \mathcal{W})\right) \times$ $\mathcal{P}(\mathcal{X})$, and $R \mapsto E_{\mathrm{s}}(R)$ is continuous on $\left[H(P)-, I_{\infty}^{\mathrm{a}, *}(P, \mathcal{W}), H(P)-, I_{0}^{\mathrm{a}}(P, \mathcal{W})\right)$.

Thirdly, Proposition 16 below shows that the entropic exponent for i.i.d. source coding with QSI can be reproduced by the type-dependent source; see [55].

Proposition 16 (Entropic Duality in Source Coding with Quantum Side Information [55]). Let $\rho_{X B}=$ $\sum_{x \in \mathcal{X}} P_{X}(x)|x\rangle\langle x| \otimes \rho_{B}^{x} \in \mathcal{S}(X B)$ be a joint state of a classical source coding with quantum side information. For any $R \geq 0$, the following holds:

$$
E_{\mathrm{S}}(R)=\min _{Q \in \mathcal{P}(\mathcal{X})}\left\{E_{\mathrm{s}}(R, Q)+D\left(Q \| P_{X}\right)\right\}
$$

Lastly, using the concavity of the auxiliary functions given in Section 5, Fenchel's duality theorem [125] directly yields the following useful duality representation in joint source-channel coding with QSI [56]. 
Proposition 17 (Fenchel Duality in Joint Source-Channel Coding with Quantum Side Information [56]). Consider a joint source-channel coding with a classical-quantum joint state $\rho_{X B} \in \mathcal{S}(X B)$ and a classicalquantum channel $\mathcal{W}: \mathcal{X} \rightarrow \mathcal{S}(\mathcal{H})$. Let $Q \in \mathcal{P}(\mathcal{X})$ and denote by $\sigma_{X B}:=\sum_{x \in \mathcal{X}} Q(x)|x\rangle\langle x| \otimes \rho_{B}^{x}$. Provided $H(X \mid B)_{\sigma}<I(Q, \mathcal{W})$, we have

$$
\sup _{s \geq 0}\left\{E_{0, \mathrm{~s}}(s, Q)+E_{0}^{a}(s, Q)\right\}=\inf _{H(X \mid B)_{\sigma}<R<I(Q, \mathcal{W})}\left\{E_{\mathrm{s}}(R, Q)+E_{\mathrm{c}}(R, Q)\right\} .
$$

On the other hand, for $H(X \mid B)_{\sigma}>I(Q, \mathcal{W})$,

$$
\sup _{-1<s<0}\left\{E_{0, \mathrm{~s}}^{*}(s, Q)+E_{0}^{a, *}(s, Q)\right\}=\inf _{I(Q, \mathcal{W})<R<H(X \mid B)_{\sigma}}\left\{E_{\mathrm{s}}(R, Q)+E_{\mathrm{c}}(R, Q)\right\} .
$$

We remark that Proposition 17 is a main ingredient to establish the strong converse exponent in joint source-channel coding with QSI [56]

Proof of Proposition 17. We first recall Fenchel's duality theorem [125] named after Werner Fenchel. Let $f$ (resp. $g$ ) be proper convex function (resp. proper concave function) from some Banach space to extended real lines. Then,

$$
\inf _{x}\{f(x)-g(x)\}=\sup _{x^{*}}\left\{g_{*}\left(x^{*}\right)-f^{*}\left(x^{*}\right)\right\}
$$

where

$$
f^{*}\left(x^{*}\right):=\sup _{x}\left\{\left\langle x^{*}, x\right\rangle-f(x)\right\}
$$

is the convex conjugate of $f$, and $\langle\cdot, \cdot\rangle$ denotes a inner product. Similarly,

$$
g_{*}\left(x^{*}\right):=\inf _{x}\left\{\left\langle x^{*}, x\right\rangle-g(x)\right\}
$$

is the concave conjugate of $g$.

In view of the definitions given above, we let $x=s, x^{*}=R,-f(x)=E_{0, \mathrm{~s}}(s, Q)$ for $s \geq 0$, or $-f(x)=E_{0, \mathrm{~s}}^{*}(s, Q)$ for $s \in(-1,0)$, and let $g(x)=E_{0}^{\mathrm{a}}(s, P)$ for $s \geq 0$, or $g(x)=E_{0}^{\mathrm{a}, *}(s, P)$ for $s \in(-1,0)$. It can be verified that $f^{*}\left(x^{*}\right)=E_{\mathrm{s}}(R, Q)$ and $-g_{*}\left(x^{*}\right)=E_{\mathrm{c}}(R, Q)$. Then, it suffices to show that the auxiliary functions are concave in $s$. The concavity of $E_{0, \mathrm{~s}}$ and $E_{0}^{\mathrm{a}}$ on $s \geq 0$ follows from [86, Proposition B.5]. The concavity of $E_{0, \mathrm{~s}}^{*}$ and $E_{0}^{\mathrm{a}, *}$ on $s \in(-1,0)$ follows from Theorem 11, which completes the proof.

\section{Conclusions and Open Problems}

We study the Rényi information and Augustin information defined via the Petz, sandwiched, and the log-Euclidean Rényi divergences. The uniform equicontinuity and the convexity/concavity in prior probability distributions were proved. For various quantum auxiliary functions, we established the joint continuity in order and prior. Moreover, we solve the open problems of the concavity in the region of $s \in(-1,0)$ by employing the complex interpolation theory. The established properties allow us to better understand the entropic exponents of c-q channel coding, classical data compression with quantum side information, and joint source-channel coding with quantum side information. Applications include a minimax identity in the strong converse region, an entropic duality, and a Fenchel duality relation. We list the following open problems in the case of infinite-dimensional Hilbert spaces.

- Does the Augustin mean uniquely exist for the Petz and the sandwiched form?

- Are $I_{\alpha}^{\mathrm{r},(t)}$ and $I_{\alpha}^{\mathrm{a},(t)}$ continuous at $\alpha=1$ from below for $(t)=\{\}$, b, or $*$ ? (Note that we only know that $\alpha \mapsto I_{\alpha}^{r}$ is analytical due to the noncommutative Sibson's identity given in Proposition 2.)

- Are $I_{\alpha}^{\mathrm{r},(t)}$ and $I_{\alpha}^{\mathrm{a},(t)}$ continuously differentiable at $\alpha=1$ for $(t)=\{\} b$ or $*$ ? For example, as proven in the commuting case by Nakiboğlu [36, Lemma 17]., we conjecture that

$$
\left.\frac{\partial}{\partial \alpha} I_{\alpha}^{\mathrm{a},(t)}(P, \mathcal{W})\right|_{\alpha=z}=\frac{\partial}{\partial \alpha} D_{\alpha}^{(t)}\left(\mathcal{W} \| q_{z, p}^{(t)} \mid P\right)
$$

where $q_{z, p}^{(t)}$ is the associated Augustin mean.

- Are $E_{0}^{\mathrm{a}}(s, P)$ and $E_{0}^{\mathrm{a}, *}(s, P)$ continuous for $s \in(-1,0)$. 
Lastly, we remark that although the Augustin information and sandwiched Rényi information do not have closed-form expressions, they can be numerically computed in finite-dimensional Hilbert spaces by existing algorithms; see e.g. [126].

\section{ACKNOWLEDGMENTS}

H.-C. Cheng is supported by the Young Scholar Fellowship (Einstein Program) of the Ministry of Science and Technology (MOST) in Taiwan under grant number MOST 109-2636-E-002-001 and 110-2636-E-002009, and is supported by the Yushan Young Scholar Program of the Ministry of Education (MOE) in Taiwan under grant number NTU-109V0904 and NTU-110V0904. H.-C. Cheng would like to thank the Center for Quantum Science and Engineering, National Taiwan University, and Physics Division, National Center for Theoretical Sciences, Taiwan (R.O.C.) for their supports. We would like to thank Milán Mosonyi and Tomohiro Ogawa for introducing us to the problem and the detailed discussions. We also thank Marius Junge, Nilanjana Datta, Eric P. Hanson, and Gilles Pisier for the insightful discussions. We thank Masahito Hayashi for pointing out his work [127] for the approach of using the pinching argument. Lastly, we especially thank Barış Nakiboğlu for inspiring us how Hölder's inequality comes into the play in the proof of the concavity of the auxiliary functions, for reading the early version of the manuscript, and for providing useful comments. We also thank two anonymous reviewers and the editor for carefully reviewing our paper.

\section{Appendix A. Proofs of Properties of Rényi Information and Augustin Information}

Proposition 4 (Properties of Rényi Information). Let $\mathcal{W} \subset \mathcal{S}(\mathcal{H})$, and let $(t)$ be any of the three values: \{\}$, *$ or $b$.

(a) For any $P \in \mathcal{P}(\mathcal{W}), I_{\alpha}^{\mathrm{r},(t)}(P, \mathcal{W})$ is non-negative and nondecreasing in $\alpha$. Moreover, $I_{\alpha}^{r,(t)}(P, \mathcal{W}) \leq$ $\log |\operatorname{supp}(P)|]$.

(b) The map $P \mapsto I_{\alpha}^{\mathrm{r},(t)}(P, \mathcal{W})$ is quasi-concave on $\mathcal{P}(\mathcal{W})$ for $\alpha \in[0,1)$, and concave on $\mathcal{P}(\mathcal{W})$ for $\alpha \in[1, \infty]$.

(c) Let

$$
\mathcal{A}:=[0,1], \quad \mathcal{A}^{*}:=[1 / 2, \infty], \text { and } \mathcal{A}^{b}:=[0, \infty] .
$$

For $\eta \in[0, \infty]$, if $C_{\eta, \mathcal{W}}^{(t)}<\infty$, then $\left\{I_{\alpha}^{\mathrm{r},(t)}(P, \mathcal{W})\right\}_{\alpha \in[0, \eta] \cap \mathcal{A}^{(t)}}$ is uniformly equicontinuous in $P \in$ $\mathcal{P}(\mathcal{W})$.

Proof of Proposition 4-(a). The assertion about the monotonicity follows directly from Lemma 1-(a) and the definition of $I_{\alpha}^{r,(t)}$ given in Eq. (20), which was also pointed out by Mosonyi and Ogawa [86, Lemma 4.6].

We move on to prove the upper bound. Recall Eq. (10), it suffices to prove it for Petz's version. By quantum Sibson's identity [87], it holds for every $\alpha \in(1, \infty)$,

$$
\begin{aligned}
I_{\alpha}^{\mathrm{r}}(P, \mathcal{W}) & =\frac{\alpha}{\alpha-1} \log \operatorname{Tr}\left[\left(\sum_{\omega \in \mathcal{W}} P(\omega) \omega^{\alpha}\right)^{\frac{1}{\alpha}}\right] \\
& \leq \log \operatorname{Tr}\left[\left(\sum_{\omega \in \mathcal{W}} P(\omega) \omega^{\alpha}\right)^{\frac{1}{\alpha}}\right] .
\end{aligned}
$$

Next, we employ a generalized Ando-Zhan theorem proved by Bhatia and Kittaneh [128], [129, Theorem 5]: for any positive operators $A_{1}, \ldots A_{m}$ and every unitarily invariant norm $\|\cdot\| \|$,

$$
\left\|\left(\sum_{i=1}^{m} A_{i}\right)^{r}\right\|\|\leq\| \sum_{i=1}^{m} A_{i}^{r}\|\|, \quad \forall r \in[0,1] .
$$


Therefore, we have

$$
\begin{aligned}
I_{\alpha}^{\mathrm{r}}(P, \mathcal{W}) & \leq \log \operatorname{Tr}\left[\sum_{\omega \in \mathcal{W}} P(\omega)^{\frac{1}{\alpha} \omega}\right] \\
& \leq \log |\operatorname{supp}(P)|,
\end{aligned}
$$

which completes the proof.

Proof of Proposition 4-(b). The arguments follow similar from [74, Theorems 7, 8]. Note that for every $\sigma \in \mathcal{S}(\mathcal{H})$

$$
D_{\alpha}(P \circ \mathcal{W} \| P \otimes \sigma)=\frac{1}{\alpha-1} \log \sum_{\omega \in \mathcal{W}} P(\omega) \mathrm{e}^{(\alpha-1) D_{\alpha}^{(t)}(\omega \| \sigma)} .
$$

Since $\frac{1}{\alpha} \log (\alpha)$ is a decreasing function for $\alpha \in[0,1)$ and concave function for $\alpha \geq 1$, the assertions follow because pointwise infimum of quasiconcave functions is concave.

Proof of Proposition 4-(c). Let $t$ be any of three values, and fix any $\alpha \in \mathcal{A}^{(t)}$. We prove our claim by showing for any $P_{1}, P_{2} \in \mathcal{P}(\mathcal{X})$,

$$
\begin{aligned}
& \sup _{\alpha \in[0, \eta] \cap \mathcal{A}^{(t)}}\left|I_{\alpha}^{\mathrm{r},(t)}\left(P_{2}, \mathcal{W}\right)-I_{\alpha}^{\mathrm{r},(t)}\left(P_{1}, \mathcal{W}\right)\right| \\
& \leq \begin{cases}\log \left[\frac{1}{1-\delta} \wedge \frac{\mathrm{e}^{C_{0, \mathcal{W}}^{(t)}}}{\delta}\right]^{(t)}+\log \left[1-\delta+\delta \mathrm{e}^{\left.C_{0, \mathcal{W}}^{(t)}\right],}\right. & \eta=0 \\
\log \frac{1-\delta+\delta \mathrm{e}^{C_{\eta, \mathcal{W}}}}{\left[(1-\delta)^{\frac{1}{\eta}}+\delta^{\frac{1}{\eta}} \mathrm{e}^{\frac{\eta-1}{\eta}} C_{\eta, \mathcal{W}}^{(t)}\right]^{\frac{\eta}{1-\eta}}}, & \eta \in \mathbb{R}_{>0} \backslash 1 \\
h(\delta)+\delta C_{1, \mathcal{W}}+\log \left[1-\delta+\delta \mathrm{e}^{C_{1, \mathcal{W}}}\right], & \eta=1\end{cases}
\end{aligned}
$$

where $\delta:=\frac{1}{2}\left\|P_{1}-P_{2}\right\|_{1}$ and $h(\delta):=-\delta \log \delta-(1-\delta) \log (1-\delta)$. We remark that the presentation follows from the commuting case in Ref. [38] for readers' convenience.

Invoke the decomposition provided in [38, Lemma 4-(c)], i.e.

$$
\begin{aligned}
& P_{1}=(1-\delta) s_{\wedge}+\delta s_{1}, \\
& P_{2}=(1-\delta) s_{\wedge}+\delta s_{2},
\end{aligned}
$$

where $s_{\wedge}:=\frac{P_{1} \wedge P_{2}}{1-\delta}, s_{1}:=\frac{P_{1}-P_{1} \wedge P_{2}}{\delta}, s_{2}:=\frac{P_{2}-P_{1} \wedge P_{2}}{\delta}$. For notational convenience, we let $Q_{\alpha}^{(t)}(\rho \| \sigma):=$ $\mathrm{e}^{(\alpha-1) D_{\alpha}^{(t)}(\rho \| \sigma)}$. Further, we denote the Rényi mean for any distribution $P \in \mathcal{P}(\mathcal{X})$ and $\alpha>0$ by

$$
\sigma_{\alpha, P} \in \underset{\sigma \in \mathcal{S}(\mathcal{H})}{\arg \min } D_{\alpha}^{(t)}(P \circ \mathcal{W} \| P \otimes \sigma) .
$$

We begin the proof by showing a lower bound on $I_{\alpha}^{\mathrm{r},(t)}\left(P_{1}, \mathcal{W}\right)$. Note that the order-1 Rényi mean $\sigma_{1, P}$ equals to the average state $P \mathcal{W}$ (see e.g. [130]). Direct calculation shows that

$$
\begin{aligned}
I_{1}\left(P_{1}, \mathcal{W}\right) & =(1-\delta) I_{1}\left(s_{\wedge}, \mathcal{W}\right)+(1-\delta) D_{1}\left(\sigma_{1, s_{\wedge}} \| \sigma_{1, P_{1}}\right)+\delta I_{1}\left(s_{1}, \mathcal{W}\right)+\delta D_{1}\left(\sigma_{1, s_{1}} \| \sigma_{1, P_{1}}\right) \\
& \geq(1-\delta) I_{1}\left(s_{\wedge}, \mathcal{W}\right)+\delta I_{1}\left(s_{1}, \mathcal{W}\right),
\end{aligned}
$$

where the inequality follows from the non-negativity of the Rényi divergence given in Lemma 1-(b).

Using Eq. (42), it follows that for $\alpha \neq 1$,

$$
\begin{aligned}
I_{\alpha}^{r,(t)}\left(P_{1}, \mathcal{W}\right) & =\frac{1}{\alpha-1} \log \left[(1-\delta) \sum_{\omega} s_{\wedge}(\omega) Q_{\alpha}^{(t)}\left(\omega \| \sigma_{\alpha, P_{1}}\right)+\delta \sum_{\omega} s_{1}(\omega) Q_{\alpha}^{(t)}\left(\omega \| \sigma_{\alpha, P_{1}}\right)\right] \\
& \geq \frac{1}{\alpha-1} \log \left[(1-\delta) \sum_{\omega} s_{\wedge}(\omega) Q_{\alpha}^{(t)}\left(\omega \| \sigma_{\alpha, s_{\wedge}}\right)+\delta \sum_{x} s_{1}(\omega) Q_{\alpha}^{(t)}\left(\omega \| \sigma_{\alpha, s_{1}}\right)\right],
\end{aligned}
$$


where the inequality follows from the definition of $I_{\alpha}^{\mathrm{r},(t)}$ given in Eq. (20), i.e.

$$
\begin{aligned}
& I_{\alpha}^{r,(t)}\left(s_{\wedge}, \mathcal{W}\right)=\frac{1}{\alpha-1} \log \sum_{\omega} s_{\wedge}(\omega) Q_{\alpha}^{(t)}\left(\omega \| \sigma_{\alpha, s_{\wedge}}\right) \leq \frac{1}{\alpha-1} \log \sum_{\omega} s_{\wedge}(\omega) Q_{\alpha}^{(t)}\left(\omega \| \sigma_{\alpha, P_{1}}\right), \\
& I_{\alpha}^{r,(t)}\left(s_{1}, \mathcal{W}\right)=\frac{1}{\alpha-1} \log \sum_{\omega} s_{1}(\omega) Q_{\alpha}^{(t)}\left(\omega \| \sigma_{\alpha, s_{1}}\right) \leq \frac{1}{\alpha-1} \log \sum_{\omega} s_{1}(\omega) Q_{\alpha}^{(t)}\left(\omega \| \sigma_{\alpha, P_{1}}\right) .
\end{aligned}
$$

Then, from Eqs. (43) and (44), we have

$$
\begin{aligned}
I_{\alpha}^{r,(t)}\left(P_{1}, \mathcal{W}\right) & \geq \begin{cases}(1-\delta) I_{1}\left(s_{\wedge}, \mathcal{W}\right)+\delta I_{1}\left(s_{1}, \mathcal{W}\right), & \alpha=1 \\
\frac{1}{\alpha-1} \log \left[(1-\delta) \mathrm{e}^{(\alpha-1) I_{\alpha}^{\mathrm{r},(t)}\left(s_{\wedge}, \mathcal{W}\right)}+\delta \mathrm{e}^{\left.(\alpha-1) I_{\alpha}^{\mathrm{r},(t)}\left(s_{1}, \mathcal{W}\right)\right],}\right. & \alpha \neq 1\end{cases} \\
& =I_{\alpha}^{r,(t)}\left(s_{\wedge}, \mathcal{W}\right)-g\left(\delta, \alpha, I_{\alpha}^{r,(t)}\left(s_{\wedge}, \mathcal{W}\right)-I_{\alpha}^{r,(t)}\left(s_{1}, \mathcal{W}\right)\right),
\end{aligned}
$$

where for any $\delta \in[0,1], \alpha>0, \gamma \in \mathbb{R}$, we define the function $g(\delta, \alpha, \gamma)$ by

$$
g(\delta, \alpha, \gamma):=\left\{\begin{array}{ll}
\delta \gamma, & \alpha=1 \\
\frac{1}{1-\alpha} \log \left[(1-\delta)+\delta \mathrm{e}^{(1-\alpha) \gamma}\right], & \alpha \neq 1
\end{array} .\right.
$$

Since $\alpha \mapsto g(\delta, \alpha, \gamma)$ is nonincreasing (see [38, p. 25]), we have

$$
I_{\alpha}^{\mathrm{r},(t)}\left(P_{1}, \mathcal{W}\right) \geq I_{\alpha}^{\mathrm{r},(t)}\left(s_{\wedge}, \mathcal{W}\right)-g\left(\delta, 0, I_{\alpha}^{\mathrm{r},(t)}\left(s_{\wedge}, \mathcal{W}\right)-I_{\alpha}^{\mathrm{r},(t)}\left(s_{1}, \mathcal{W}\right)\right) .
$$

Moreover, the map $\gamma \mapsto g(\delta, \alpha, \gamma)$ is nondecreasing. Hence, by using $I_{\alpha}^{\mathrm{r},(t)}\left(s_{1}, \mathcal{W}\right) \geq 0, I_{\alpha}^{\mathrm{r},(t)}\left(s_{\wedge}, \mathcal{W}\right) \leq$ $I_{\eta}^{\mathrm{r},(t)}\left(s_{\wedge}, \mathcal{W}\right)$, and $I_{\eta}^{\mathrm{r},(t)}\left(s_{1}, \mathcal{W}\right) \leq C_{\eta, \mathcal{W}}^{(t)}$, we obtain

$$
I_{\alpha}^{\mathrm{r},(t)}\left(P_{1}, \mathcal{W}\right) \geq I_{\alpha}^{\mathrm{r},(t)}\left(s_{\wedge}, \mathcal{W}\right)-g\left(\delta, 0, C_{\eta, \mathcal{W}}^{(t)}\right)
$$

Next, we move on to show an upper bound on $I_{\alpha}^{\mathrm{r},(t)}\left(P_{2}, \mathcal{W}\right)$. For $\alpha=1$, we have

$$
\begin{aligned}
I_{1}\left(P_{2}, \mathcal{W}\right) & =\inf _{\sigma \in \mathcal{S}(\mathcal{H})} \sum_{\omega \in \mathcal{W}} P(\omega) D(\omega \| \sigma) \\
& \leq \sum_{\omega \in \mathcal{W}} P(\omega) D\left(\omega \|(1-\delta) \sigma_{1, s_{\wedge}}+\delta \sigma_{1, s_{2}}\right) \\
& =(1-\delta) I_{1}\left(s_{\wedge}, \mathcal{W}\right)+\delta I_{1}\left(s_{2}, \mathcal{W}\right)+h(\delta) .
\end{aligned}
$$

On the other hand, from the definition of $I_{\alpha}^{r,(t)}$ given in Eq. (20) and using Eq. (42) again, we have, for any $\alpha \neq 1$ and $\sigma \in \mathcal{S}(\mathcal{H})$,

$$
\begin{aligned}
I_{\alpha}^{\mathbf{r},(t)}\left(P_{2}, \mathcal{W}\right) & \leq D_{\alpha}^{(t)}\left(P_{2} \circ \mathcal{W} \| P_{2} \otimes \sigma\right) \\
& =\frac{1}{\alpha-1} \log \left[(1-\delta) \sum_{x} s_{\wedge}(x) Q_{\alpha}^{(t)}(\omega \| \sigma)+\delta \sum_{x} s_{2}(x) Q_{\alpha}^{(t)}(\omega \| \sigma)\right] .
\end{aligned}
$$

Here, we choose

$$
\sigma=\frac{\theta \sigma_{\alpha, s_{\wedge}}+\vartheta \sigma_{\alpha, s_{2}}}{\theta+\vartheta} \in \mathcal{S}(\mathcal{H}), \theta:=(1-\delta)^{\frac{1}{\alpha}} \mathrm{e}^{\frac{\alpha-1}{\alpha} I_{\alpha}^{\mathrm{r},(t)}\left(s_{\wedge}, \mathcal{W}\right)}, \vartheta:=\delta^{\frac{1}{\alpha}} \mathrm{e}^{\frac{\alpha-1}{\alpha} I_{\alpha}^{\mathrm{r},(t)}\left(s_{2}, \mathcal{W}\right)} .
$$

Note that $\theta, \vartheta \geq 0$. Hence,

$$
\sigma \geq \frac{\theta}{\theta+\vartheta} \sigma_{\alpha, s_{\wedge}}, \text { and } \sigma \geq \frac{\vartheta}{\theta+\vartheta} \sigma_{\alpha, s_{2}}
$$

Lemma 1-(c) implies that $Q_{\alpha}^{(t)}$ is nonincreasing in its second argument for $\alpha>1$ and nondecreasing in its second argument for $\alpha<1$. Using this fact and combining Eqs. (46), (47) and (48), direct calculation 
yields

$$
\begin{aligned}
I_{\alpha}^{\mathbf{r},(t)}\left(P_{2}, \mathcal{W}\right) & \leq \begin{cases}(1-\delta) I_{1}\left(s_{\wedge}, \mathcal{W}\right)+\delta I_{1}\left(s_{2}, \mathcal{W}\right)+h(\delta), & \alpha=1 \\
\frac{\alpha}{\alpha-1} \log [\theta+\vartheta], & \alpha \neq 1\end{cases} \\
& =I_{\alpha}^{r,(t)}\left(s_{\wedge}, \mathcal{W}\right)+f\left(\delta, \alpha, I_{\alpha}^{r,(t)}\left(s_{2}, \mathcal{W}\right)-I_{\alpha}^{r,(t)}\left(s_{\wedge}, \mathcal{W}\right)\right),
\end{aligned}
$$

where for any $\delta \in[0,1], \alpha>0, \gamma \in \mathbb{R}$, we define the function $f(\delta, \alpha, \gamma)$ by

$$
f(\delta, \alpha, \gamma):=\left\{\begin{array}{ll}
\delta \gamma+h(\delta), & \alpha=1 \\
\frac{\alpha}{\alpha-1} \log \left[(1-\delta)^{\frac{1}{\alpha}}+\delta^{\frac{1}{\alpha}} \mathrm{e}^{\frac{\alpha-1}{\alpha} \gamma}\right], & \alpha \neq 1
\end{array} .\right.
$$

Since $\alpha \mapsto f(\delta, \alpha, \gamma)$ is nondcreasing (see [38, p. 26]), we have

$$
I_{\alpha}^{\mathrm{r},(t)}\left(P_{2}, \mathcal{W}\right) \leq I_{\alpha}^{\mathrm{r},(t)}\left(s_{\wedge}, \mathcal{W}\right)+f\left(\delta, \eta, I_{\alpha}^{\mathrm{r},(t)}\left(s_{2}, \mathcal{W}\right)-I_{\alpha}^{\mathrm{r},(t)}\left(s_{\wedge}, \mathcal{W}\right)\right) .
$$

Further, the map $\gamma \mapsto g(\delta, \alpha, \gamma)$ is nondecreasing. Hence, by using $I_{\alpha}^{\mathrm{r},(t)}\left(s_{\wedge}, \mathcal{W}\right) \geq 0, I_{\alpha}^{\mathrm{r},(t)}\left(s_{2}, \mathcal{W}\right) \leq$ $I_{\eta}^{\mathrm{r},(t)}\left(s_{2}, \mathcal{W}\right)$, and $I_{\eta}^{\mathrm{r},(t)}\left(s_{2}, \mathcal{W}\right) \leq C_{\eta, \mathcal{W}}^{(t)}$, we obtain

$$
I_{\alpha}^{\mathrm{r},(t)}\left(P_{2}, \mathcal{W}\right) \leq I_{\alpha}^{\mathrm{r},(t)}\left(s_{\wedge}, \mathcal{W}\right)+f\left(\delta, \eta, C_{\eta, \mathcal{W}}^{(t)}\right) .
$$

Combining Eqs. (45) and (49) gives

$$
I_{\alpha}^{\mathrm{r},(t)}\left(P_{2}, \mathcal{W}\right)-I_{\alpha}^{\mathrm{r},(t)}\left(P_{1}, \mathcal{W}\right) \leq f\left(\delta, \eta, C_{\eta, \mathcal{W}}^{(t)}\right)+g\left(\delta, 0, C_{\eta, \mathcal{W}}^{(t)}\right) .
$$

A lower bound on $I_{\alpha}^{\mathrm{r},(t)}\left(P_{2}, \mathcal{W}\right)-I_{\alpha}^{\mathrm{r},(t)}\left(P_{1}, \mathcal{W}\right)$ can be shown by using a similar argument and reversing the roles of $P_{1}$ and $P_{2}$.

It remains to show Eq. (41) for $\alpha=0$. We remark that the argument of this case follows from the similar ideas in [38, Lemma 16-(e)]. We provide the proof here for completeness. From the definition given in Eq. (20), we have

$$
\begin{aligned}
I_{0}^{\mathrm{r},(t)}\left(P_{1}, \mathcal{W}\right) & =-\sup _{\sigma \in \mathcal{S}(\mathcal{H})} \log \left[(1-\delta) \sum_{x} s_{\wedge}(x) Q_{0}^{(t)}(\omega \| \sigma)+\delta \sum_{x} s_{1}(x) Q_{0}^{(t)}(\omega \| \sigma)\right] \\
& \geq-\log \left[(1-\delta) \sup _{\sigma \in \mathcal{S}(\mathcal{H})} \sum_{\omega} s_{\wedge}(\omega) Q_{0}^{(t)}(\omega \| \sigma)+\delta \sup _{\sigma \in \mathcal{S}(\mathcal{H})} \sum_{\omega} s_{1}(\omega) Q_{0}^{(t)}(\omega \| \sigma)\right] \\
& =-\log \left[(1-\delta) \mathrm{e}^{-I_{0}^{\mathrm{r},(t)}\left(s_{\wedge}, \mathcal{W}\right)}+\delta \mathrm{e}^{-I_{0}^{\mathrm{r},(t)}\left(s_{1}, \mathcal{W}\right)}\right] \\
& \geq-\log \left[(1-\delta) \mathrm{e}^{-I_{0}^{\mathrm{r},(t)}\left(s_{\wedge}, \mathcal{W}\right)}+\delta\right] \\
& =I_{0}^{\mathrm{r},(t)}\left(s_{\wedge}, \mathcal{W}\right)-\log \left[1-\delta+\delta \mathrm{e}^{\left.I_{0}^{\mathrm{r},(t)}\left(s_{\wedge}, \mathcal{W}\right)\right]},\right.
\end{aligned}
$$

where the inequalities follows from the subadditivity of supremum and $I_{0}^{\mathrm{r},(t)}\left(s_{1}, \mathcal{W}\right) \geq 0$.

On the other hands,

$$
\begin{aligned}
I_{0}^{\mathrm{r},(t)}\left(P_{1}, \mathcal{W}\right) & =\inf _{\sigma \in \mathcal{S}(\mathcal{H})} \log \frac{1}{(1-\delta) \sum_{\omega} s_{\wedge}(\omega) Q_{0}^{(t)}(\omega \| \sigma)+\delta \sum_{\omega} s_{2}(x) Q_{0}^{(t)}(\omega \| \sigma)} \\
& \leq\left(\inf _{\sigma \in \mathcal{S}(\mathcal{H})} \log \frac{1}{(1-\delta) \sum_{\omega} s_{\wedge}(\omega) Q_{0}^{(t)}(\omega \| \sigma)}\right) \wedge\left(\inf _{\sigma \in \mathcal{S}(\mathcal{H})} \log \frac{1}{(1-\delta) \sum_{\omega} s_{2}(\omega) Q_{0}^{(t)}(\omega \| \sigma)}\right) \\
& =\left(I_{0}^{\mathbf{r},(t)}\left(s_{\wedge}, \mathcal{W}\right)+\log \frac{1}{1-\delta}\right) \wedge\left(I_{0}^{r,(t)}\left(s_{2}, \mathcal{W}\right)+\log \frac{1}{\delta}\right) .
\end{aligned}
$$


Hence, Eqs. (50) along with (51) lead to

$$
I_{0}^{(t)}\left(P_{2}, \mathcal{W}\right)-I_{0}^{(t)}\left(P_{1}, \mathcal{W}\right) \leq \log \left[1-\delta+\delta \mathrm{e}^{C_{\eta, \mathcal{W}}^{(t)}}\right]+\log \left[\frac{1}{1-\delta} \wedge \frac{\mathrm{e}^{C_{\eta, \mathcal{W}}^{(t)}}}{\delta}\right]
$$

A lower bound on $I_{0}^{\mathrm{r},(t)}\left(P_{2}, \mathcal{W}\right)-I_{0}^{\mathrm{r},(t)}\left(P_{1}, \mathcal{W}\right)$ can be shown by using a similar argument and reversing the roles of $P_{1}$ and $P_{2}$. As a result, Eq. (41) holds for $\eta=0$ and $\alpha=0$.

Finally, the case of $\eta>0$ and $\alpha=0$ follows by noting that

$$
\frac{\eta}{\eta-1} \log \left[(1-\delta)^{\frac{1}{\eta}}+\delta^{\frac{1}{\eta}} \mathrm{e}^{\frac{\eta-1}{\eta} C_{\eta, \mathcal{W}}}\right] \geq \log \left[\frac{1}{1-\delta} \wedge \frac{\mathrm{e}^{C_{\eta, \mathcal{W}}^{(t)}}}{\delta}\right] .
$$

Proposition 5 (Properties of Augustin Information). Let $\mathcal{W} \subset \mathcal{S}(\mathcal{H})$ be a classical-quantum channel, $(t)$ be any of the three values: \{\} , *, or $b$, and let $\mathcal{A}^{(t)}$ be defined in (23).

(a) For every $P \in \mathcal{P}(\mathcal{X}), I_{\alpha}^{\mathrm{a},(t)}(P, \mathcal{W})$ is non-negative and nondecreasing in $\alpha$. Moreover, $I_{\alpha}^{\mathrm{a},(t)}(P, \mathcal{W}) \leq$ $H(P)$ for $\alpha \in \mathcal{A}^{(t)}$, where $H(P)$ is the Shannon entropy of $P$.

(b) For any $\alpha>0$, the map $P \mapsto I_{\alpha}^{\mathrm{a},(t)}(P, \mathcal{W})$ is concave on $\mathcal{P}(\mathcal{W})$.

(c) Let $\mathcal{A}^{(t)}$ be defined in (40). For $\eta \in[0, \infty]$, if $C_{\eta, \mathcal{W}}^{(t)}<\infty$, then $\left\{I_{\alpha}^{a,(t)}(P, \mathcal{W})\right\}_{\alpha \in(0, \eta] \cap \mathcal{A}^{(t)}}$ is uniformly equicontinuous in $P \in \mathcal{P}(\mathcal{W})$.

Proof of Proposition 5-(a). As in Proposition 4-(a), the assertion about the monotonicity follows direct from Lemma (a) and the definition of $I_{\alpha}^{\mathrm{a},(t)}$ given in Eq. (21).

The upper bound follows similar idea as in [36, Lemma 13]. The definition of $I_{\alpha}^{\mathrm{a},(t)}$ implies that

$$
\begin{aligned}
I_{\alpha}^{\mathrm{a},(t)}(P, \mathcal{W}) & =\inf _{\sigma \in \mathcal{S}(\mathcal{H})} \sum_{\omega} P(\omega) D_{\alpha}^{(t)}(\omega \| \sigma) \\
& \leq \sum_{\omega} P(\omega) D_{\alpha}^{(t)}(\omega \| P \mathcal{W}) \\
& \leq \sum_{\omega} P(\omega) \log \frac{1}{P(\omega)}
\end{aligned}
$$

where the last inequality follows from Lemma 1-(c).

Proof of Proposition 5-(b). The concavity immediately follows from the definition of $I_{\alpha}^{\mathrm{a},(t)}$ given in Eq. (21), and the fact that pointwise infimum of concave functions is concave.

Proof of Proposition 5-(c). Fix $t$ be any of the three values. To prove the equicontinuity, we need the following inequality:

$$
I_{\alpha}^{\mathrm{a},(t)}\left(\alpha, P_{\beta}\right) \leq \beta I_{\alpha}^{\mathrm{a},(t)}\left(\alpha, P_{1}\right)+(1-\beta) I_{\alpha}^{\mathrm{a},(t)}\left(\alpha, P_{0}\right)+H(\beta)
$$

for any $P_{1}, P_{0} \in \mathcal{P}(\mathcal{X}), P_{\beta}=\beta P_{1}+(1-\beta) P_{0}, \beta \in(0,1), \alpha \in[0,1] ;$ and we shorthand $H(\beta):=$ $-\beta \log \beta-(1-\beta) \log (1-\beta)$ the binary entropy function.

We denote the Augustin mean for any distribution $P \in \mathcal{P}(\mathcal{X})$ and $\alpha>0$ by

$$
\sigma_{\alpha, P} \in \underset{\sigma \in \mathcal{S}(\mathcal{H})}{\arg \min } \sum_{\omega \in \mathcal{W}} P(\omega) D_{\alpha}^{(t)}(\omega \| \sigma)
$$


Lemma 1-(b) implies that, for every $\alpha \in[0,1]$,

$$
\begin{aligned}
& \sum_{\omega \in \mathcal{W}} P_{\beta}(\omega) D_{\alpha}^{(t)}\left(\omega \| \beta \sigma_{\alpha, P_{1}}+(1-\beta) \sigma_{\alpha, P_{0}}\right) \\
& =\beta \sum_{\omega \in \mathcal{W}} P_{1}(\omega) D_{\alpha}^{(t)}\left(\omega \| \beta \sigma_{\alpha, P_{1}}+(1-\beta) \sigma_{\alpha, P_{0}}\right)+(1-\beta) \sum_{\omega \in \mathcal{W}} P_{0}(\omega) D_{\alpha}^{(t)}\left(\omega \| \beta \sigma_{\alpha, P_{1}}+(1-\beta) \sigma_{\alpha, P_{0}}\right) \\
& \leq \beta \sum_{\omega \in \mathcal{W}} P_{1}(\omega) D_{\alpha}^{(t)}\left(\omega \| \sigma_{\alpha, P_{1}}\right)-\beta \log \beta+(1-\beta) \sum_{\omega \in \mathcal{W}} P_{0}(\omega) D_{\alpha}^{(t)}\left(\omega \| \sigma_{\alpha, P_{0}}\right)-(1-\beta) \log (1-\beta) \\
& =\beta I_{\alpha}^{\mathrm{a},(t)}\left(P_{1}, \mathcal{W}\right)+(1-\beta) I_{\alpha}\left(P_{0}, \mathcal{W}\right)+H(\beta) .
\end{aligned}
$$

Let $s_{\wedge}, s_{1}, s_{0}$ be

$$
\begin{aligned}
& s_{\wedge}=\frac{P_{1} \wedge P_{0}}{\left\|P_{1} \wedge P_{0}\right\|_{1}}, \\
& s_{1}=\frac{P_{1}-P_{1} \wedge P_{0}}{1-\left\|P_{1} \wedge P_{0}\right\|_{1}}, \\
& s_{0}=\frac{P_{0}-P_{1} \wedge P_{0}}{1-\left\|P_{1} \wedge P_{0}\right\|_{1}} .
\end{aligned}
$$

One can verify that.

$$
\begin{aligned}
& P_{1}=\left(1-\frac{\left\|P_{1}-P_{0}\right\|_{1}}{2}\right) s_{\wedge}+\frac{\left\|P_{1}-P_{0}\right\|_{1}}{2} s_{1}, \\
& P_{0}=\left(1-\frac{\left\|P_{1}-P_{0}\right\|_{1}}{2}\right) s_{\wedge}+\frac{\left\|P_{1}-P_{0}\right\|_{1}}{2} s_{0} .
\end{aligned}
$$

Then, the concavity of $P \mapsto I_{\alpha}^{\mathrm{a},(t)}(P, \mathcal{W})$ given in item (b) together with Eq. (52) yield

$$
\begin{aligned}
I_{\alpha}^{\mathrm{a},(t)}\left(P_{0}, \mathcal{W}\right)-I_{\alpha}^{\mathrm{a},(t)}\left(P_{1}, \mathcal{W}\right) & \leq H\left(\frac{\left\|P_{1}-P_{0}\right\|_{1}}{2}\right)+\frac{\left\|P_{1}-P_{0}\right\|_{1}}{2}\left(I_{\alpha}^{\mathrm{a},(t)}\left(s_{0}, \mathcal{W}\right)-I_{\alpha}^{\mathrm{a},(t)}\left(s_{1}, \mathcal{W}\right)\right) \\
& \leq H\left(\frac{\left\|P_{1}-P_{0}\right\|_{1}}{2}\right)+\frac{\left\|P_{1}-P_{0}\right\|_{1}}{2} I_{\alpha}^{\mathrm{a},(t)}\left(s_{0}, \mathcal{W}\right)
\end{aligned}
$$

for $\alpha \geq 0$. Thus, using the monotone increases of $\alpha \mapsto I_{\alpha}^{\mathrm{a},(t)}$ given in (a) and recalling the definition of Rényi capacity given in Eq. (22),

$$
\left|I_{\alpha}^{\mathrm{a},(t)}\left(P_{0}, \mathcal{W}\right)-I_{\alpha}^{\mathrm{a},(t)}\left(P_{1}, \mathcal{W}\right)\right| \leq H\left(\frac{\left\|P_{1}-P_{0}\right\|_{1}}{2}\right)+\frac{\left\|P_{1}-P_{0}\right\|_{1}}{2} C_{\eta, \mathcal{W}} .
$$

The above inequality implies equicontinuity as desired.

\section{REFERENCES}

[1] H. Chernoff, "A measure of asymptotic efficiency for tests of a hypothesis based on the sum of observations," The Annals of Mathematical Statistics, vol. 23, no. 4, pp. 493-507, dec 1952.

[2] - "Large-sample theory: Parametric case," The Annals of Mathematical Statistics, vol. 27, no. 1, pp. 1-22, mar 1956.

[3] W. Hoeffding, "Probability inequalities for sums of bounded random variables," Journal of the American Statistical Association, vol. 58, no. 301, p. 13, mar 1963.

[4] — "On probabilities of large deviations," in Proceedings of the Fifth Berkeley Symposium on Mathematical Statistics and Probability, Volume 1: Statistics. Berkeley, Calif.: University of California Press, 1967, pp. 203-219. [Online]. Available: https://projecteuclid.org/euclid.bsmsp/1200512987

[5] E. L. Lehmann, Testing Statistical Hypotheses. Springer New York, 1986.

[6] R. R. Bahadur, "Rates of convergence of estimates and test statistics," The Annals of Mathematical Statistics, vol. 38, no. 2, pp. 303-324, apr 1967.

[7] — Some Limit Theorems in Statistics. Society for Industrial and Applied Mathematics, jan 1971.

[8] R. Bahadur, "Large deviations of the maximum likelihood estimate in the Markov chain case." in Recent Advances in Statistics. Elsevier, 1983, pp. 273-286. 
[9] A. D. M. Kester and W. C. M. Kallenberg, "Large deviations of estimators," The Annals of Statistics, vol. 14, no. 2, pp. 648-664, jun 1986.

[10] I. M. Chakravarti, Ed., Asymptotic Theory of Statistical Tests and Estimation: In Honor of Wassily Hoeffding. Academic Press, 1980.

[11] T. Berger, Rate distortion theory: A mathematical basis for data compression. Prentice-Hall, 1971.

[12] J. O. Berger, Statistical Decision Theory. Springer New York, 1980.

[13] A. Dembo and O. Zeitouni, Large Deviations Techniques and Applications. Springer, 1998.

[14] C. E. Shannon, "A mathematical theory of communication," The Bell System Technical Journal, vol. 27, pp. 379-423, 1948.

[15] A. Feinstein, "Error bounds in noisy channels without memory," IEEE Transactions on Information Theory, vol. 1, no. 2, pp. 13-14, Sep 1955.

[16] C. E. Shannon, "Probability of error for optimal codes in a Gaussian channel," Bell System Technical Journal, vol. 38, no. 3, pp. 611-656, May 1959.

[17] R. M. Fano, Transmission of Information, A Statistical Theory of Communications. The MIT Press, 1961.

[18] R. Gallager, "A simple derivation of the coding theorem and some applications," IEEE Transaction on Information Theory, vol. 11, no. 1, pp. 3-18, Jan 1965.

[19] — Information Theory and Reliable Communication. Wiley, 1968.

[20] C. Shannon, R. Gallager, and E. Berlekamp, "Lower bounds to error probability for coding on discrete memoryless channels. I," Information and Control, vol. 10, no. 1, pp. 65-103, Jan 1967.

[21] — - "Lower bounds to error probability for coding on discrete memoryless channels. II," Information and Control, vol. 10, no. 5, pp. 522-552, May 1967.

[22] S. Arimoto, "On the converse to the coding theorem for discrete memoryless channels," IEEE Transactions on Information Theory, vol. 19, no. 3, pp. 357-359, may 1973.

[23] G. Dueck and J. Körner, "Reliability function of a discrete memoryless channel at rates above capacity (corresp.)," IEEE Transactions on Information Theory, vol. 25, no. 1, pp. 82-85, jan 1979.

[24] Y. Polyanskiy and S. Verdu, "Arimoto channel coding converse and Rényi divergence," in 2010 48th Annual Allerton Conference on Communication, Control, and Computing (Allerton). IEEE, sep 2010.

[25] B. Nakiboğlu, "The sphere packing bound via Augustin's method," IEEE Transactions on Information Theory, vol. 65, no. 2, pp. 816-840, feb 2019.

[26] I. Csiszár and J. Körner, Information Theory: Coding Theorems for Discrete Memoryless Systems. Cambridge University Press (CUP), 2011.

[27] R. Sibson, "Information radius," Zeitschrift für Wahrscheinlichkeitstheorie und Verwandte Gebiete, vol. 14, no. 2, pp. 149-160, 1969.

[28] A. Rényi, "On measures of entropy and information," Proc. 4th Berkeley Symp. on Math. Statist. Probability, vol. 1, pp. 547-561, 1961.

[29] T. van Erven and P. Harremoes, "Rényi divergence and kullback-leibler divergence," IEEE Transactions on Information Theory, vol. 60, no. 7, pp. 3797-3820, jul 2014.

[30] U. Augustin, "Noisy channels," 1978, habilitation thesis, Universitat Erlangen.

[31] I. Csiszár, "Generalized cutoff rates and Rényi's information measures," IEEE Transactions on Information Theory, vol. 41, no. 1, pp. 26-34, 1995.

[32] S. Arimoto, "Information measures and capacity of order $\alpha$ for discrete memoryless channels," Colloquia Mathematica Societatis Jańos Bolya, vol. 16, pp. 41-52, 1977.

[33] S. Verdú, " $\alpha$-mutual information," in 2015 Information Theory and Applications Workshop (ITA). IEEE, feb 2015.

[34] G. Aishwarya and M. Madiman, "Conditional Rényi entropy and the relationships between Rényi capacities," Entropy, vol. 22, no. 5, p. 526, may 2020.

[35] M. Dalai, "Some remarks on classical and classical-quantum sphere packing bounds: Rényi vs. Kullback-Leibler," Entropy, vol. 19, no. 7, p. 355, jul 2017.

[36] B. Nakiboğlu, "The Augustin capacity and center," Problems of Information Transmission, vol. 55, no. 4, pp. 299-342, oct 2019 .

[37] J. Kemperman, "On the shannon capacity of an arbitrary channel," Indagationes Mathematicae (Proceedings), vol. 77, no. 2, pp. 101-115, 1974.

[38] B. Nakiboğlu, "The Rényi capacity and center," IEEE Transactions on Information Theory, vol. 65, no. 2, pp. 841-860, feb 2019.

[39] R. E. Blahut, "Hypothesis testing and information theory," IEEE Transaction on Information Theory, vol. 20, no. 4, pp. 405-417, Jul 1974.

[40] — Principles and practice of information theory. Addison-Wesley, 1987.

[41] U. Augustin, "Gedächtnisfreie kanäle für diskrete zeit," Zeitschrift für Wahrscheinlichkeitstheorie und Verwandte Gebiete, vol. 6, no. 1, pp. 10-61, 1966.

[42] — - "Error estimates for low rate codes," Zeitschrift für Wahrscheinlichkeitstheorie und Verwandte Gebiete, vol. 14, no. 1, pp. 61-88, 1969.

[43] E. A. Haroutunian, "Estimates of the error exponents for the semicontinuous memoryless channel," Problemy Peredachi Informatsii, vol. 4, no. 4, pp. 37-48, 1968, (in Russian). [Online]. Available: http://mi.mathnet.ru/eng/ppi1871 
[44] E. A. Haroutunian, M. E. Haroutunian, and A. N. Harutyunyan, "Reliability criteria in information theory and in statistical hypothesis testing," Foundations and Trends(R) in Communications and Information Theory, vol. 4, no. 2-3, pp. 97-263, 2007.

[45] R. G. Gallager, "Fixed composition arguments and lower bounds to error probability," (unpublished) 1994. [Online]. Available: http://web.mit.edu/gallager/www/notes/notes5.pdf

[46] I. Csiszár and J. Körner, "Graph decomposition: A new key to coding theorems," IEEE Transactions on Information Theory, vol. 27, no. 1, pp. 5-12, Jan 1981.

[47] I. Csiszar, "The method of types," IEEETransactions on Information Theory, vol. 44, no. 6, pp. 2505-2523, 1998.

[48] J. Scarlett, A. Martinez, and A. Guillén i F'abregas, "Mismatched decoding: Error exponents, second-order rates and saddlepoint approximations," IEEE Transactions on Information Theory, vol. 60, no. 5, pp. 2647-2666, May 2014.

[49] J. Scarlett, "Reliable communication under mismatched decoding," 2014, phD thesis (University of Cambridge).

[50] B. Nakiboğlu, "The sphere packing bound for memoryless channels," Problems of Information Transmission, vol. 56, no. 3, pp. 201-244, jul 2020.

[51] M. Hayashi, "Error exponent in asymmetric quantum hypothesis testing and its application to classical-quantum channel coding," Physical Review A, vol. 76, no. 6, Dec 2007.

[52] H.-C. Cheng, M.-H. Hsieh, and M. Tomamichel, "Quantum sphere-packing bounds with polynomial prefactors," IEEE Transactions on Information Theory, vol. 65, no. 5, pp. 2872-2898, may 2019.

[53] H.-C. Cheng and M.-H. Hsieh, "Moderate deviation analysis for classical-quantum channels and quantum hypothesis testing," IEEE Transactions on Information Theory, vol. 64, no. 2, pp. 1385-1403, feb 2018.

[54] H.-C. Cheng, E. P. Hanson, N. Datta, and M.-H. Hsieh, "Non-asymptotic classical data compression with quantum side information," IEEE Transactions on Information Theory, vol. 67, no. 2, pp. 902-930, feb 2021.

[55] — - "Duality between source coding with quantum side information and c-q channel coding," 2018, arXiv:1809.11143.

[56] — - "Non-asymptotic joint source-channel coding with quantum side informations," (in preparation).

[57] D. Slepian and J. Wolf, "Noiseless coding of correlated information sources," IEEE Transactions on Information Theory, vol. 19, no. 4, pp. 471-480, Jul 1973.

[58] R. G. Gallager, "Source coding with side information and universal coding," 1976, Technical Report. [Online]. Available: http://web.mit.edu/gallager/www/papers/paper5.pdf

[59] V. N. Košhelev, "On a problem of separate coding of two dependent sources," Problems of Information Transmission, vol. 13, no. 1, pp. 18-22, Mar 1977, (In Russian). [Online]. Available: http://mi.mathnet.ru/eng/ppi1064

[60] I. Csiszár and J. Körner, "Towards a general theory of source networks," IEEE Transactions on Information Theory, vol. 26, no. 2, pp. 155-165, Mar 1980.

[61] I. Csiszar, "Linear codes for sources and source networks: Error exponents, universal coding," IEEE Transactions on Information Theory, vol. 28, no. 4, pp. 585-592, Jul 1982.

[62] R. Ahlswede, "Coloring hypergraphs: A new approach to multi-user source coding, 2," Journal of combinatorics, information 83 system sciences, vol. 5, no. 3, pp. 220-268, 1980.

[63] R. Ahlswede and G. Dueck, "Good codes can be produced by a few permutations," IEEE Transactions on Information Theory, vol. 28, no. 3, pp. 430-443, may 1982.

[64] R. Ahlswede, Storing and Transmitting Data, A. Ahlswede, I. Althöfer, C. Deppe, and U. Tamm, Eds. Springer International Publishing, 2014.

[65] I. Csiszár, "Information-type measures of difference of probability distributions and indirect observations," Studia Scientiarum Mathematicarum Hungarica, vol. 2, pp. 299-318, 1967.

[66] Y. Altuğ and A. B. Wagner, "Moderate deviations in channel coding," IEEE Transactions on Information Theory, vol. 60, no. 8, pp. 4417-4426, Aug 2014.

[67] Y. Polyanskiy, H. V. Poor, and S. Verdu, "Channel coding rate in the finite blocklength regime," IEEE Trans. Inform. Theory, vol. 56, no. 5, pp. 2307-2359, May 2010.

[68] V. Y. F. Tan, "Asymptotic estimates in information theory with non-vanishing error probabilities," Foundations and Trends (R) in Communications and Information Theory, vol. 10, no. 4, pp. 1-184, 2014.

[69] J. Scarlett, A. Martinez, and A. Guillén i F'abregas, "The saddlepoint approximation: Unified random coding asymptotics for fixed and varying rates," in 2014 IEEE International Symposium on Information Theory. Institute of Electrical and Electronics Engineers (IEEE), Jun 2014.

[70] Y. Altuğ and A. B. Wagner, "Refinement of the random coding bound," IEEE Transactions on Information Theory, vol. 60, no. 10, pp. 6005-6023, oct 2014.

[71] D. J. H. Garling, Inequalities: A Journey into Linear Analysis. Cambridge University Press, 2007.

[72] A. Ben-Tal, M. Teboulle, and A. Charnes, "The role of duality in optimization problems involving entropy functionals with applications to information theory," Journal of Optimization Theory and Applications, vol. 58, no. 2, pp. 209-223, Aug 1988.

[73] Y. Altuğ and A. B. Wagner, "Refinement of the sphere-packing bound: Asymmetric channels," IEEE Transactions on Information Theory, vol. 60, no. 3, pp. 1592-1614, Mar 2014.

[74] S.-W. Ho and S. Verdú, "Convexity/concavity of Rényi entropy and $\alpha$-mutual information," in 2015 IEEE International Symposium on Information Theory (ISIT). IEEE, jun 2015.

[75] M. A. Nielsen and I. L. Chuang, Quantum Computation and Quantum Information. Cambridge University Press, 2009 . 
[76] M. Hayashi, Quantum Information: An Introduction. Springer.

[77] M. M. Wilde, Quantum Information Theory. Cambridge University Press, 2016.

[78] M. Tomamichel, Quantum Information Processing with Finite Resources. Springer International Publishing, 2016.

[79] H.-C. Cheng, "Error exponent analysis in quantum information theory," PhD Thesis (University of Technology Sydney), 2018.

[80] D. Petz, "Quasi-entropies for finite quantum systems," Reports on Mathematical Physics, vol. 23, no. 1, pp. 57-65, Feb 1986.

[81] M. Müller-Lennert, F. Dupuis, O. Szehr, S. Fehr, and M. Tomamichel, "On quantum Rényi entropies: A new generalization and some properties," Journal of Mathematical Physics, vol. 54, no. 12, p. 122203, 2013.

[82] M. M. Wilde, A. Winter, and D. Yang, "Strong converse for the classical capacity of entanglement-breaking and Hadamard channels via a sandwiched Rényi relative entropy," Communications in Mathematical Physics, vol. 331, no. 2, pp. 593-622, Jul 2014.

[83] M. Berta, V. B. Scholz, and M. Tomamichel, "Rényi divergences as weighted non-commutative vector-valued $L_{p^{-}}$ spaces," Annales Henri Poincaré, vol. 19, no. 6, pp. 1843-1867, mar 2018.

[84] A. Jenčová, "Rényi relative entropies and noncommutative $L_{p}$-spaces," Annales Henri Poincaré, vol. 19, no. 8, pp. 2513-2542, jun 2018.

[85] T. Ogawa and H. Nagaoka, "Strong converse and Stein's lemma in quantum hypothesis testing," IEEE Transaction on Information Theory, vol. 46, no. 7, pp. 2428-2433, 2000.

[86] M. Mosonyi and T. Ogawa, "Strong converse exponent for classical-quantum channel coding," Communications in Mathematical Physics, vol. 355, no. 1, pp. 373-426, Oct 2017.

[87] N. Sharma and N. A. Warsi, "Fundamental bound on the reliability of quantum information transmission," Physical Review Letters, vol. 110, no. 8, Feb 2013.

[88] M. V. Burnashev and A. S. Holevo, "On the reliability function for a quantum communication channel," Problems of information transmission, vol. 34, no. 2, pp. 97-107, 1998.

[89] A. Holevo, "Reliability function of general classical-quantum channel," IEEE Transaction on Information Theory, vol. 46, no. 6, pp. 2256-2261, 2000.

[90] M. Hayashi and H. Nagaoka, "General formulas for capacity of classical-quantum channels," IEEE Transaction on Information Theory, vol. 49, no. 7, pp. 1753-1768, Jul 2003.

[91] H. Qi, Q. Wang, and M. M. Wilde, "Applications of position-based coding to classical communication over quantum channels," Journal of Physics A: Mathematical and Theoretical, 2018.

[92] A. Winter, "Coding theorems of quantum information theory," 1999, (PhD Thesis, Universität Bielefeld).

[93] M. Dalai, "Lower bounds on the probability of error for classical and classical-quantum channels," IEEE Transactions on Information Theory, vol. 59, no. 12, pp. 8027-8056, Dec 2013.

[94] M. Dalai and A. Winter, "Constant conpositions in the sphere packing bound for classical-quantum channels," IEEE Transactions on Information Theory, vol. 63, no. 9, Sept 2017.

[95] S. Golden, "Lower bounds for the Helmholtz function," Physical Review, vol. 137, no. 4B, pp. B1127-B1128, Feb 1965.

[96] K. Symanzik, "Proof and refinements of an inequality of Feynman," Journal of Mathematical Physics, vol. 6, no. 7, pp. 1155-1156, jul 1965.

[97] C. J. Thompson, "Inequality with applications in statistical mechanics," Journal of Mathematical Physics, vol. 6, no. 11, p. 1812, 1965.

[98] H.-C. Cheng and M.-H. Hsieh, "Concavity of the auxiliary function for classical-quantum channels," IEEE Transactions on Information Theory, vol. 62, no. 10, pp. 5960 - 5965, 2016.

[99] M. Junge and J. Parcet, "Mixed-norm inequalities and operator space $L_{p}$ embedding theory," Memoirs of the American Mathematical Society, vol. 203, no. 953, 2010.

[100] G. Pisier, "Non-commutative vector valued $L_{p}$-spaces and completely p-summing maps," Asterisque Societe Mathematique de France, vol. 247, 1998.

[101] M. Sion, "On general minimax theorems," Pacific Journal of Mathematics, vol. 8, no. 1, pp. 171-176, mar 1958.

[102] M. Mosonyi, "Coding theorems for compound problems via quantum Rényi divergences," IEEE Transactions on Information Theory, vol. 61, no. 6, pp. 2997-3012, jun 2015.

[103] H. Umegaki, "Conditional expectation in an operator algebra. IV. entropy and information," Kodai Mathematical Seminar Reports, vol. 14, no. 2, pp. 59-85, 1962.

[104] S. Beigi, "Sandwiched Rényi divergence satisfies data processing inequality," Journal of Mathematical Physics, vol. 54, no. 12, p. 122202, 2013.

[105] E. H. Lieb and W. E. Thirring, "Inequalities for the moments of the eigenvalues of the Schrodinger Hamiltonian and their relation to Sobolev inequalities." Walter de Gruyter GmbH.

[106] H. Araki, "On an inequality of Lieb and Thirring," Letters in Mathematical Physics, vol. 19, no. 2, pp. 167-170, feb 1990.

[107] M. Tomamichel and M. Hayashi, "A Hierarchy of Information Quantities for Finite Block Length Analysis of Quantum Tasks," IEEE Transactions on Information Theory, vol. 59, no. 11, pp. 7693-7710, Nov. 2013, 00112 arXiv: 1208.1478.

[108] K. Li, "Second-order asymptotics for quantum hypothesis testing," The Annals of Statistics, vol. 42, no. 1, pp. 171-189, Feb 2014.

[109] J. Bergh and J. Löfström, Interpolation Spaces. Springer Berlin Heidelberg, 1976. 
[110] J.-C. Bourin and E.-Y. Lee, "Matrix inequalities from a two variables functional," International Journal of Mathematics, vol. 27, no. 09, p. 1650071, Aug 2016.

[111] J. Shao, "Two variables functionals and inequalities related to measurable operators," Journal of Inequalities and Applications, vol. 2017, no. 1, dec 2017.

[112] A. Holevo, "The capacity of the quantum channel with general signal states," IEEE Transaction on Information Theory, vol. 44, no. 1, pp. 269-273, 1998.

[113] M. Junge, "Doob's inequality for non-commutative martingales," Journal fur die Reine und Angewandte Mathematik, pp. 149-190, 2002.

[114] M. Junge and Q. Xu, "Noncommutative maximal ergodic theorems," Journal of the American Mathematical Society, vol. 20, no. 2, pp. 385-439, 2007.

[115] U. Haagerup, " $L_{p}$-spaces associated with an arbitrary von Neumann algebra," in Algebres d'opérateurs et leurs applications en physique mathématique (Proc. Colloq., Marseille, 1977), vol. 274, 1979, pp. 175-184.

[116] T. Fack and H. Kosaki, "Generalized s-numbers of $\tau$-measurable operators," Pacific Journal of Mathematics, vol. 123, no. 2, pp. 269-300, jun 1986.

[117] H. Kosaki, "Applications of the complex interpolation method to a von Neumann algebra: Non-commutative $L_{p}$ spaces," Journal of Functional Analysis, vol. 56, no. 1, pp. 29-78, mar 1984.

[118] É. Ricard, "Hölder estimates for the noncommutative Mazur maps," Archiv der Mathematik, vol. 104, no. 1, pp. 37-45, 2015 .

[119] A. Devinatz, "The factorization of operator valued functions," Annals of Mathematics, pp. 458-495, 1961.

[120] G. Pisier, "Factorization of operator valued analytic functions," Advances in Mathematics, vol. 93, no. 1, pp. 61-125, 1992.

[121] É. Ricard, "Hölder estimates for the noncommutative Mazur maps," Archiv der Mathematik, vol. 104, no. 1, pp. 37-45, dec 2014.

[122] M. Mosonyi and T. Ogawa, "Divergence radii and the strong converse exponent of classical-quantum channel coding with constant compositions," IEEE Transactions on Information Theory, vol. 67, no. 3, pp. 1668-1698, mar 2021.

[123] C. Berge, Topological Spaces. Oliver \& Boyd, 1963.

[124] B. Pshenichnyi, Necessary Conditions for an Extremum Pshenichnyi. CRC Press, 1971.

[125] R. T. Rockafellar, Convex Analysis. Walter de Gruyter GmbH, Jan 1970.

[126] J.-K. You, H.-C. Cheng, and Y.-H. Li, "Minimizing quantum Rényi divergences via mirror descent with Polyak step size," 2021, arXiv:2109.06054.

[127] M. Hayashi and M. Tomamichel, "Correlation detection and an operational interpretation of the Rényi mutual information," Journal of Mathematical Physics, vol. 57, no. 10, p. 102201, Oct 2016.

[128] T. Ando and X. Zhan, "Norm inequalities related to operator monotone functions," Mathematische Annalen, vol. 315, no. 4, pp. 771-780, dec 1999.

[129] R. Bhatia and F. Kittaneh, "Clarkson inequalities with several operators," Bulletin of the London Mathematical Society, vol. 36, no. 06, pp. 820-832, oct 2004.

[130] B. Schumacher and M. D. Westmoreland, "Sending classical information via noisy quantum channels," Physical Review A, vol. 56, no. 1, pp. 131-138, Jul 1997. 